\title{
Predictions for boson-jet observables and fragmentation function ratios from a hybrid strong/weak coupling model for jet quenching
}

\author{
Jorge Casalderrey-Solana, ${ }^{a}$ Doga Can Gulhan, ${ }^{b}$ José Guilherme Milhano, ${ }^{c, d}$ \\ Daniel Pablos $^{a}$ and Krishna Rajagopal ${ }^{b, e}$ \\ ${ }^{a}$ Departament d'Estructura $i$ Constituents de la Matèria \\ and Institut de Ciències del Cosmos (ICCUB), Universitat de Barcelona, \\ Martí i Franquès 1, 08028 Barcelona, Spain \\ ${ }^{b}$ Laboratory for Nuclear Science and Department of Physics, \\ Massachusetts Institute of Technology (MIT), \\ Cambridge, MA 02139 U.S.A. \\ ${ }^{c}$ CENTRA, Instituto Superior Técnico, Universidade de Lisboa, \\ Av. Rovisco Pais, P-1049-001 Lisboa, Portugal \\ ${ }^{d}$ Physics Department, Theory Unit, CERN, \\ CH-1211 Genève 23, Switzerland \\ ${ }^{e}$ Center for Theoretical Physics, MIT, \\ Cambridge, MA 02139, U.S.A. \\ E-mail: jorge.casalderrey@ub.edu, dgulhan@mit.edu, \\ guilherme.milhano@tecnico.ulisboa.pt, dpablos@ecm.ub.es, \\ krishna@mit.edu
}

ABSTRACT: We have previously introduced a hybrid strong/weak coupling model for jet quenching in heavy ion collisions in which we describe the production and fragmentation of jets at weak coupling, using PYTHIA, and describe the rate at which each parton in the jet shower loses energy as it propagates through the strongly coupled plasma, $d E / d x$, using an expression computed holographically at strong coupling. The model has a single free parameter that we fit to a single experimental measurement. We then confront our model with experimental data on many other jet observables, focusing in this paper on boson-jet observables, finding that it provides a good description of present jet data. Next, we provide the predictions of our hybrid model for many measurements to come, including those for inclusive jet, dijet, photon-jet and Z-jet observables in heavy ion collisions with energy $\sqrt{s}=5.02 \mathrm{ATeV}$ coming soon at the LHC. As the statistical uncertainties on near-future measurements of photon-jet observables are expected to be much smaller than 
those in present data, with about an order of magnitude more photon-jet events expected, predictions for these observables are particularly important. We find that most of our preand post-dictions do not depend sensitively on the form we choose for the rate of energy loss $d E / d x$ of the partons in the shower. This gives our predictions considerable robustness. To better discriminate between possible forms for the rate of energy loss, though, we must turn to intrajet observables. Here, we focus on ratios of fragmentation functions. We close with a suggestion for a particular ratio, between the fragmentation functions of inclusive and associated jets with the same kinematics in the same collisions, which is particularly sensitive to the $x$ - and $E$-dependence of $d E / d x$, and hence may be used to learn which mechanism of parton energy loss best describes the quenching of jets.

Keywords: Heavy Ion Phenomenology, Jets

ARXIV EPRINT: 1508.00815 


\section{Contents}

1 Introduction 1

2 Description of the model and its implementation 5

2.1 The hybrid model approach 5

2.2 The effects of flow on the rate of energy loss 8

$\begin{array}{ll}2.3 \text { The effects of flow on single-jet and dijet observables } & 11\end{array}$

$\begin{array}{lll}2.4 & \text { Species dependence of jet suppression } & 13\end{array}$

3 Boson-jet correlations, including predictions for $\sqrt{s}=5.02 \mathrm{ATeV}$ colli$\begin{array}{ll}\text { sions and for Z-jet correlations } & \mathbf{1 5}\end{array}$

$\begin{array}{lll}3.1 & \text { Generation and selection of Monte Carlo events } & 17\end{array}$

3.2 Photon-jet observables: comparison with experimental results at $\sqrt{s}=$ $\begin{array}{ll}2.76 \mathrm{ATeV} \text { and predictions for } \sqrt{s}=5.02 \mathrm{ATeV} & 19\end{array}$

3.3 Z-jet observables: predictions for $\sqrt{s}=5.02 \mathrm{ATeV}$

4 Fragmentation functions $\quad \mathbf{2 7}$

4.1 Fragmentation functions of the associated jets in photon-jet and Z-jet pairs 28

4.2 Fragmentation functions of the associated jets in dijet pairs 34

$\begin{array}{lll}5 & \text { Conclusions and outlook } & 37\end{array}$

$\begin{array}{ll}\text { A Energy Loss in a boosted fluid } & 41\end{array}$

B Update on single-jet and dijet observables at $\sqrt{s}=2.76 \mathrm{ATeV} \quad 42$

C Predictions for single-jet and dijet observables at $\sqrt{s}=5.02 \mathrm{ATeV} \quad 44$

$\begin{array}{ll}\text { D Model dependence of boson-jet correlations } & 47\end{array}$

\section{Introduction}

The LHC has ushered in a new era in the exploration of the properties of matter under extreme conditions. By colliding $\mathrm{Pb}$ ions at center of mass energies in the multi- $\mathrm{TeV}$ regime, the LHC has provided us with droplets of the hottest matter ever produced in the laboratory and a diverse suite of copious high energy probes with which to explore the microscopic properties of the strongly coupled, liquid, quark-gluon plasma (QGP) discovered at RHIC [1-4]. For jet probes in particular, the results of the successful LHC Run 1 have shown that many of the properties of these fundamental QCD objects are substantially modified when the jets are produced in $\mathrm{Pb}-\mathrm{Pb}$ collisions as compared to 
when they are produced in p-p collisions [5-20]. These modifications are results of the final state interaction between the jets and the droplets of hot QCD matter formed in heavy ion collisions. As the principal underlying effect is the energy loss suffered by each of the components of the jet showers on their way out of the hot matter, the various modifications of jet properties observed in heavy ion collisions are referred to, in sum, as jet quenching. The phenomenon of jet quenching was first discovered without reconstructing individual jets via the strong reduction in the number of intermediate- $p_{\mathrm{T}}$ hadrons in heavy ion collisions at RHIC [21, 22]. Precisely because varied modifications of jet properties can now be well measured, this suite of probes has the potential to provide us with unique and important information about the properties of QGP and about the interaction between energetic partons and this strongly coupled liquid. The imminent start of the LHC heavy ion Run 2, which will increase both the center of mass energy and luminosity for heavy ion collisions and hence will substantially increase the production rates of jets and all other hard probes including high energy photons and Z-bosons, makes many much more quantitative analyses of the striking phenomena observed in the first LHC run imminent. Turning this opportunity into precise extractions of QGP properties from the experimental data to come requires a diverse suite of theoretical tools. In this paper, we shall present substantial advances in the development of one such tool, introduced in ref. [23].

It is the discovery that the QGP of QCD is a strongly coupled liquid, with intense collective phenomena, no apparent quasiparticle structure, and a very rich phenomenology that makes it of such interest. But, at the same time, it makes the theoretical description of its properties and dynamics much more challenging, since many of the perturbative tools available to describe weakly coupled hard QCD processes become inapplicable in the strongly coupled liquid plasma produced in the range of temperatures accessible to colliders. Fortunately, in recent years gauge/gravity duality has emerged as a tool with which to analyze the dynamics of the strongly coupled plasma in various non-Abelian gauge theories. Although it is not yet known whether QCD itself has a dual gravitational description, calculations done using these new holographic methods (where one maps a question in the gauge theory plasma onto a calculation done in its dual gravitational description) have yielded many qualitative insights into the properties and dynamics of the QGP in QCD, as produced and probed in heavy ion collisions. (For a review see ref. [24].)

Although we now have a toolkit that includes perturbative methods valid for weakly coupled hard processes and holographic calculations that are valuable guides to strongly coupled dynamics, the description of the interactions between jets and the plasma remains challenging because physics at both hard and soft scales enters in central ways. Jets in the $100 \mathrm{GeV}$ range are produced in hard processes with large momentum transfers which must be described by perturbative QCD. Furthermore, the energetic, virtual, partons produced in a hard scattering immediately experience a rapid sequence of branching processes which reduce their large initial virtuality, ultimately down to the hadronization scale. The evolution of this branching process is governed by the DGLAP equations and is described well by perturbation theory, since the initial virtualities of the partons in the shower that results from the branching are large. Nevertheless, when the jet fragments interact with the plasma created in $\mathrm{Pb}-\mathrm{Pb}$ collisions, soft exchanges of energy and momentum with the 
plasma constituents are governed by physics at scales of order the temperature of the medium, where a strongly coupled description is mandated. And, it is these soft interactions that are critical to understanding the modification of jets produced in $\mathrm{Pb}-\mathrm{Pb}$ collisions caused by their interaction with the plasma. This multi-scale nature of the physics of jet probes and their modification means that neither approaches that seek to treat the both the jets and their interaction with the plasma perturbatively [25-59] nor those that seek to address the entire dynamics of the quenching of energetic probes at strong coupling via gauge/gravity duality [60-78] can capture the physics at all the relevant scales correctly.

To face this challenge, in ref. [23] we introduced a phenomenological model specifically designed to treat the different dynamics arising on different scales differently, using a perturbative description where it is appropriate and deploying insights from holographic calculations where soft momentum transfers and strongly coupled physics enter. While there have been other attempts to combine results obtained from weak and strong coupling [79-85], our approach is distinct since it focusses on using two different calculational frameworks at the different energy scales where each is appropriate. In a nutshell, our model combines a perturbative description of the creation and evolution of jets as implemented in modern Monte Carlo codes, in particular in PyтніA [86], with a prescription for the mechanism by which, and consequently the rate $d E / d x$ at which, each parton in the Pythia shower loses energy that we infer from the holographic computation [77] of how energetic light quarks lose energy in the strongly coupled plasma of a gauge theory with a dual gravitational description. As described at length in ref. [23], in the model we assume that the splitting probabilities and hence the pattern of fragmentation within the jet are as in vacuum, with the only effect of the medium being the loss of energy experienced by each parton in the jet. That is, we neglect the fact that as partons lose energy this modifies the phase space for subsequent splitting, which is to say it modifies Sudakov logs, and we neglect the possibility that soft momentum exchange with the medium can induce additional splitting. We leave adding these effects to the hybrid model to future work. As presently constituted, our hybrid model contains a single parameter which controls the stopping distance of fast partons in the QCD plasma. We fitted this parameter to data in ref. [23], and then confronted this hybrid strong/weak coupling model with data on several single-jet and dijet observables, obtaining very satisfactory agreement. Nonetheless, our analysis also showed that this same set of data could be described almost as well by different assumptions for the energy loss rate $d E / d x$ of particles in plasma. This indicates that the observables considered in ref. [23] are not very sensitive to the microscopic dynamics governing the interaction between an energetic probe and the strongly coupled plasma.

In this paper, we continue our exploration of the predictions of the hybrid model that we introduced in ref. [23], and whose construction and main aspects we review briefly in section 2.1. In section 2.2 and appendix A, we improve some aspects of the implementation of the model: we incorporate the effects of the motion of the hydrodynamic fluid on the rate of energy loss, and we embed the jets in our model in the viscous hydrodynamic simulations of refs. [87, 88], rather than the inviscid hydrodynamic simulations from ref. [89] that we used in ref. [23]. We discuss the implications of these improvements for the extraction of the value of the single parameter in our model in sections 2.3 and 2.4. 
After laying this groundwork, in section 3 we use our model to analyze photon-jet and Z-jet correlations (generically, boson-jet correlations) in heavy ion collisions. These observables provide a different and complementary set of data with which to confront our model; doing so is the first of our principal goals. After specifying the details of how we generate and quench our samples of boson-jet events in section 3.1, in section 3.2 we compare the predictions of our model to presently available photon-jet data from LHC heavy ion collisions. We find that our model is just as successful here as it was at describing inclusive jet observables and dijet observables in ref. [23] and appendix B. ${ }^{1}$ These many successes give us confidence in our approach.

In section 3 and appendix D, we find that, within current experimental and theoretical uncertainties, two different control hypotheses for the form of the rate of energy loss $d E / d x$ yield almost as good descriptions of the single-jet, dijet, and photon-jet observables that we have investigated as we obtain from our hybrid strong/weak coupling model. Similarly, the gamma-jet data we consider in this work can also be described well by the perturbative treatment of ref. [47].

Therefore, these observables are not particularly effective at discriminating between rather different assumptions about the interactions between the components of the jets and the strongly coupled medium. (This observation was first made for the specific case of the dijet asymmetry observable in ref. [90].) However, we have observed various small differences among the predictions of our hybrid strong/weak coupling model and those of our two control models, which opens the possibility that the higher energy and higher statistics data from the coming LHC heavy ion Run 2 will yield more precise measurements that can be used to constrain the microscopic dynamics of how jets interact with the plasma and, ultimately, the microscopic properties of the plasma itself. With these goals in mind, and to facilitate direct comparisons to data that is coming soon, in section 3.2 we provide predictions for photon-jet observables in $\mathrm{Pb}-\mathrm{Pb}$ collisions at $\sqrt{s}=5.02 \mathrm{ATeV}$ and in section 3.3 we do the same for Z-jet observables. We include the predictions of our model for a number of single-jet and dijet observables at $\sqrt{s}=5.02 \mathrm{ATeV}$ in appendix C. We hope that near-future high-statistics measurements will make the myriad predictions of our hybrid model for heavy ion collisions at $\sqrt{s}=5.02 \mathrm{ATeV}$ among the most incisive results of this paper.

With the goal of finding observables that are more discriminating, in the sense that they are more sensitive to different assumptions about the rate of energy loss $d E / d x$ and hence are better able to give us information about the microscopic dynamics behind jet quenching, in section 4 we turn to exploring more differential observables. Before adding further physics (and further parameters) to our hybrid model, we cannot use it to describe the modification of the angular shapes of jets. We can, however, analyze fragmentation functions. In section 4.1 we study the fragmentation functions of the jets produced in association with either a photon or a Z-boson, providing predictions for the ratios of these fragmentation functions in $\mathrm{Pb}-\mathrm{Pb}$ collisions with $\sqrt{s}=5.02 \mathrm{ATeV}$ to the same fragmenta-

\footnotetext{
${ }^{1}$ In appendix B, we update our model calculations of the observables from ref. [23] to include the improved model implementation introduced in section 2.2 .
} 
tion functions in p-p collisions. These predictions are qualitative at best, however, because they are sensitive to differences between the hadronization of jets in $\mathrm{Pb}-\mathrm{Pb}$ and $\mathrm{p}-\mathrm{p}$ collisions that are not under theoretical control. And, these observables turn out not to be particularly discriminating. In section 4.2 we use the tools that we have constructed to identify a new observable that is discriminating. This observable is the ratio between the fragmentation functions of inclusive jets in $\mathrm{Pb}-\mathrm{Pb}$ collisions and the fragmentation functions of the associated (lower energy) jets from a dijet pair. Much of the uncertainty associated with hadronization should cancel in this ratio, since we nowhere need p-p reference data and since we compare the fragmentation functions of associated jets and inclusive jets with the same range of energies, in the same $\mathrm{Pb}-\mathrm{Pb}$ collisions. Furthermore, by explicit calculation we find a clear and substantial separation between the predictions of our hybrid strong/weak coupling model for this ratio and the predictions that we obtain from our two control models. And, we find a good qualitative understanding of why this separation occurs, which is to say of why this ratio is such a discriminating observable. We look forward to measurements of this observable in the coming LHC heavy ion Run 2 as these data should tell us much about the dynamics of how jets interact with the strongly coupled plasma.

In section 5 we discuss the main conclusions of this study and look ahead.

\section{Description of the model and its implementation}

\subsection{The hybrid model approach}

In this section, we briefly review the main aspects of the hybrid model that we introduced in our previous publication [23]. Our model is a response to the challenge of addressing the wide range of energy scales involved in the production of energetic quarks, gluons, photons and Z-bosons in hard processes followed by the interaction of the quarks and gluons with the strongly coupled matter formed in ultrarelativistic heavy ion collisions. Our hybrid model treats the weakly coupled short distance dynamics involved in the creation and hard evolution of jets perturbatively while at the same time using insights from holographic calculations that assume strong coupling in the treatment of the soft long distance interactions of the jet with the strongly coupled fluid created in the collision. The former processes occur at momentum scales set by the large virtuality of the elementary hard partonic interaction, which we shall denote $Q$. The latter processes involve momenta at or close to the typical scales that characterize the strongly coupled fluid, which we take to be of order the temperature $T$. For the energetic processes involved in jet production in $\mathrm{Pb}-\mathrm{Pb}$ collisions at the LHC, $Q \gg T$. We use this separation of scales to justify our explicit separation of the treatment of the relevant dynamics for each regime. We are, however, certainly not doing a systematic expansion order by order in powers of the ratio of these scales. Instead, we construct a model in which the qualitatively different physics at the widely separated scales is each treated in the most appropriate language, in so doing introducing a single model parameter that at the present stage we must fit to data.

In our model, we assume that the soft in-medium processes cannot alter either the short distance production of hard partons in elementary partonic collisions or the hard branching 
processes by which the large initial virtuality relaxes. In p-p collisions in vacuum, those two processes are both described well by perturbative QCD; in particular, the radiative branching processes are incorporated into evolution equations which are in turn the basis of the different high energy Monte Carlo event generators like PyтнiA [86], which we shall employ. The probabilistic implementation of this evolution describes the formation of a shower of partons initiated from the initial hard process, with splitting probabilities dictated by perturbative QCD. In the environment created in a heavy ion collision, as this parton shower develops its constituents are continuously exchanging momentum with the matter produced in the collision. In our model, we assume that the splitting probabilities are not modified by these soft exchanges. This does not mean, though, that the shower remains unchanged. As a consequence of their soft exchanges of momenta with the medium, the particles in the shower lose energy and pick up momentum transverse to their original direction as they propagate through the strongly coupled liquid. In this publication, as in ref. [23], we focus entirely on the loss of energy of the partons in the shower, as we can do so in a model with only a single free parameter. In a future publication we shall introduce the transverse momentum kicks, at the expense of adding at least one more parameter. Here, as in ref. [23], we shall only describe experimental observables that are sensitive to the loss of energy by the partons in the shower and insensitive to the transverse momentum kicks that they also experience. Since the momentum transfers between the shower partons and the medium are not large, the physics of energy loss must be described at strong coupling. After assigning a life-time $\tau=2 E / Q^{2}$, with $Q$ and E the virtuality and energy of the parton, to each parton in the shower according to a formation time argument $^{2}[23,91]$, we know the points in spacetime where the parton on each branch of the shower is formed, and splits. We model the energy loss of each parton in the shower as a continuous process, supplementing the in-medium evolution with an explicit energy loss rate $d E / d x$ that models the strongly coupled dynamics of parton energy loss. We do not track what becomes of the energy lost by each parton in the shower, implicitly assuming that the lost energy is incorporated into the strongly coupled fluid, ultimately becoming soft hadrons with momenta of order $T$ that we do not model. The form that we assume for the rate of energy loss $d E / d x$ therefore fully encodes all the strongly coupled in-medium dynamics incorporated in our model.

In our model, we explore the consequences of an energy loss rate $d E / d x$ whose form is that appropriate for the rate of energy loss of an energetic massless quark (excitation in the fundamental color representation) traversing a slab of plasma with temperature $T$ and thickness $x$ in the strongly coupled plasma of $\mathcal{N}=4$ supersymmetric Yang-Mills (SYM) theory [77],

$$
\left.\frac{d E}{d x}\right|_{\text {strongly coupled }}=-\frac{4}{\pi} E_{\text {in }} \frac{x^{2}}{x_{\text {stop }}^{2}} \frac{1}{\sqrt{x_{\text {stop }}^{2}-x^{2}}}, \quad x_{\text {stop }}=\frac{1}{2 \kappa_{\mathrm{sc}}} \frac{E_{\text {in }}^{1 / 3}}{T^{4 / 3}},
$$

\footnotetext{
${ }^{2}$ This expression may be understood as the formation time of a parton of virtuality $Q$ in its rest frame, $1 / Q$, boosted to the laboratory frame by the boost factor $E / Q$. The prefactor is fixed in ref. [91] by demanding that for a very soft splitting this scale coincides with the well known expression for the formation time of a soft gluon $\tau=2 \omega / k_{\perp}^{2}$.
} 
obtained via the gauge/gravity duality. Here, $E_{\text {in }}$ is the initial energy that the massless quark has before it enters the plasma, $E(x)$ is the energy that it has after traversing the slab of thickness $x$, and $x_{\text {stop }}$ is the stopping distance of the high energy excitation - the smallest slab thickness that results in the energetic excitation losing all of its energy within the slab of plasma. In $\mathcal{N}=4 \mathrm{SYM}$ theory, the dimensionless constant $\kappa_{\mathrm{sc}}$ appearing in the expression for $x_{\text {stop }}$ is determined explicitly in terms of the 't Hooft coupling $\lambda$ and is $\kappa_{\mathrm{sc}}=1.05 \lambda^{1 / 6}[67,72,75]$. The premise of our hybrid model is that the form of $d E / d x$ in the strongly coupled quark-gluon plasma of QCD is the same as in (2.1); we shall see that this hypothesis is uncontradicted by many and varied sets of data. However, there is no reason at all to expect that the relationship between $\kappa_{\mathrm{sc}}$ and $\lambda$ should be the same in QCD and $\mathcal{N}=4$ SYM theory, as the strongly coupled plasmas of the two theories have different, and differently many, microscopic degrees of freedom. Furthermore, there are ambiguities in the definition of jets in $\mathcal{N}=4 \mathrm{SYM}$ theory: since hard processes in this theory do not produce jets [65, 92], different theoretical calculations have been developed in which highly energetic colored excitations are formed in different ways - no one of which is preferred over others as a model for jets in QCD since none is model for jet production in QCD. And, the proportionality constant between $E_{\text {in }}^{1 / 3} / T^{4 / 3}$ and $x_{\text {stop }}$ can depend on details of the particular way in which a highly energetic colored excitation is formed. For both these reasons, and as discussed in more detail in ref. [23], in our model we will assume that any differences between $d E / d x$ in the strongly coupled plasmas of QCD and $\mathcal{N}=4 \mathrm{SYM}$ theory can be absorbed in the value of $\kappa_{\mathrm{sc}}$, which we therefore take as a free parameter whose value must be fixed by fitting to data. We will refer to the form (2.1) for $d E / d x$ as strongly coupled energy loss. Our hybrid model constitutes applying this prescription for energy loss branch-by-branch to the partons in a shower that described in vacuum by PүтніA. We shall specify the implementation of our hybrid model fully in subsequent subsections.

In order to have some other benchmarks against which to compare the success of our hybrid model, as in ref. [23] we will also explore two other quite different forms for the energy loss rate $d E / d x$, one inspired by perturbative calculations of radiative energy loss and the other by perturbative calculations of collisional energy loss. These expressions are given by

$$
\left.\frac{d E}{d x}\right|_{\text {radiative }}=-\kappa_{\mathrm{rad}} T^{3} x,\left.\quad \frac{d E}{d x}\right|_{\text {collisional }}=-\kappa_{\text {coll }} T^{2}
$$

where we shall again treat $\kappa_{\text {rad }}$ and $\kappa_{\text {coll }}$ as parameters to be fixed by fitting to data. This oversimplified treatment of radiative and collisional energy loss is not meant to supersede other much more sophisticated analysis of these mechanisms [50, 93-98]. Our only goal is to use them as benchmark expressions for $d E / d x$ with very different dependence (or lack thereof) on $x$ and $E_{\text {in }}$ to that found in (2.1).

Note that we shall take (2.1) and (2.2) as rates of energy loss for quarks in the parton shower; gluons lose more energy. In the case of (2.2), gluons lose more energy by a factor of $C_{A} / C_{F}=9 / 4$ and we shall take $\kappa_{\text {rad }}$ and $\kappa_{\text {coll }}$ to be larger by this factor for gluons in the parton shower. In the case of $(2.1), x_{\text {stop }}$ is shorter for gluons than quarks by a factor 
of $\left(C_{A} / C_{F}\right)^{1 / 3}$ [64], and as in ref. [23] we shall therefore take $\kappa_{\mathrm{sc}}$ to be larger for gluons in the parton shower than for quarks by a factor of $(9 / 4)^{1 / 3}$.

The energy loss rates eqs. (2.1) and (2.2) were derived to describe the degradation of the energy of partons traversing a static plasma with some constant temperature $T$. In reality, the strongly coupled liquid created in a heavy ion collision is finite in spatial extent and expands and cools rapidly, meaning that $T$ depends strongly on both position and time. We will nevertheless use these expressions for the instantaneous $d E / d x$, to describe the energy lost by the partons in a shower as they traverse the expanding cooling strongly coupled matter and ultimately the energy lost by the jets that emerge from the debris of the heavy ion collision. We describe our implementation of this approach in the next section.

\subsection{The effects of flow on the rate of energy loss}

In the previous section, we have specified the rate of energy loss for an energetic parton traversing static plasma with some constant temperature $T$. In order to use these expressions in the description of how an energetic parton loses energy as it traverses the expanding, flowing, cooling, plasma created in a heavy ion collision, we will exploit the fact that the expansion, flow and cooling of this fluid are described well by nearly inviscid hydrodynamics. This implies that the dynamic medium can be described as a collection of fluid cells that are each close to thermal equilibrium, locally. As is standard in fluid mechanics, local thermal equilibrium should be understood from a coarse-grained point of view: at every fluid cell there is a macroscopic system of size much larger than any microscopic scale, such as the inverse temperature of the cell. From this coarse-grained perspective, the temperature and the velocity of the fluid cell change from point to point, and from time to time.

Accounting for the variation in the temperature of the fluid is straightforward, and was already incorporated in our previous publication [23]. We assume that the temperature $T$ appearing in the formulae (2.1) or (2.2) for $d E / d x$ varies in space and time, and at each point in spacetime passed by the energetic parton is given by the temperature of the fluid, in the local fluid rest frame, at that point in spacetime. The basic assumption behind this adiabatic prescription is that the length scale on which an infinitesimal energy loss occurs is small compared to the length scale over which $T$ changes. In ref. [23], we took the variation in temperature into account by integrating (2.1) or (2.2) along the trajectory of each parton in the shower in the collision center-of-mass frame, taking $T$ at each point along the trajectory from the hydrodynamic solution describing the bulk fluid.

The prescription employed in ref. [23] does not take the velocity of the hydrodynamic fluid fully into consideration, as in this prescription the velocity of the moving fluid only affects $d E / d x$ in so far as it results in changes in the temperature of the fluid. There is an additional effect, that we chose to neglect for simplicity in ref. [23] but shall incorporate here. It is seen most simply by considering a fluid with a constant temperature flowing with a uniform velocity. It is clear from their derivations that (2.1) or (2.2) describe the rate of energy loss of an energetic parton moving through this fluid in the local fluid rest frame. If this is not the collision center-of-mass frame, $d E / d x$ in that frame must be obtained via a Lorentz transformation. In this paper we will incorporate this effect into our model 
description and, in so doing, will improve upon our previous treatment of the effects of the medium dynamics on the loss of energy of partons in a jet. Not surprisingly, we will find that incorporating the effects of fluid flow on the rate of energy loss has significant effects on our results at large rapidity, since it is at large rapidity that the boost between the local fluid rest frame and the collision center-of-mass frame becomes large.

Let us denote the rate of energy loss in the local fluid rest frame by

$$
\frac{d E_{F}}{d x_{F}}=\mathcal{F}_{F}\left(x_{F}, E_{\text {in }}^{F}\right)
$$

where the function $\mathcal{F}_{F}\left(x_{F}, E_{\text {in }}^{F}\right)$ is given by the right-hand side of (2.1) or (2.2) and where we have highlighted in the notation that $d E_{F} / d x_{F}$ depends upon the distance $x_{F}$ that the parton has travelled in the local fluid rest frame and, in the case of (2.1), upon the initial energy $E_{\text {in }}^{F}$ that the parton had when it was produced at a splitting point in the shower, again as evaluated in the local fluid rest frame. In making this statement, we have assumed that the effects of the spatial and temporal gradients in the fluid on $d E_{F} / d x_{F}$ can be neglected. ${ }^{3}$ We now Lorentz transform the rate of energy loss (2.3) back to the collision center-of-mass frame, obtaining a result that we shall denote by

$$
\frac{d E}{d x}=\mathcal{F}\left(t, E_{\text {in }}\right)
$$

where $t$ is the time in the collision center-of-mass frame since the parton was produced and $E_{\text {in }}$ is the energy that the parton had, in that frame, when it was produced. We can change from $x$ to $t$ at will because throughout our treatment we are assuming that the energetic partons in the shower move at (very close to) the speed of light. The functions $\mathcal{F}_{F}$ and $\mathcal{F}$ are related explicitly by a Lorentz transformation that we perform in appendix A. The result takes on the surprisingly simple form

$$
\left.\mathcal{F}\left(x, E_{\text {in }}\right)=\mathcal{F}_{F}\left(x_{F}, E_{\text {in }}^{F}(E)\right)\right)
$$

where $E_{\text {in }}^{F}$ and $x_{F}$ are the initial energy and the path length in the local fluid rest frame. These are related to quantities defined in the collision center-of-mass frame by

$$
\begin{aligned}
E_{\mathrm{in}}^{F} & =E_{\mathrm{in}} \gamma_{F}(1-\mathbf{w} \mathbf{v}), \\
x_{F}(t) & =\int_{t_{0}}^{t} d t \sqrt{\left[\mathbf{w}^{2}+\gamma_{F}^{2}\left(\mathbf{v}^{2}-2 \mathbf{v} \mathbf{w}+(\mathbf{v w})^{2}\right)\right]},
\end{aligned}
$$

where $\mathbf{w} \equiv \mathbf{p} / E$ is the parton velocity, $\mathbf{v}$ and $\gamma_{F}$ are the fluid velocity and Lorentz factor, $t_{0}$ the time the parton was produced and $t$ is the observation time, all in the collision center-of-mass frame. The derivations of these expressions are also given in appendix A.

\footnotetext{
${ }^{3}$ The effects of spatial and temporal gradients in the fluid on the rate of energy loss of an infinitely heavy quark moving through strongly coupled plasma have been computed, to lowest order in fluid gradients [99101]. They can be significant early in a collision, before hydrodynamization. Once the fluid is hydrodynamic, these effects are small. We expect that the same is true for the effects of fluid gradients on the rate of energy loss of a massless parton moving through the plasma, but we defer checking this by explicit calculation to future work.
} 
The result (2.5) implies that if the rate of energy loss does not depend explicitly on the energy of the parton or the distance that the parton has travelled through the medium, as in the case of the collisional rate in (2.2), the fluid velocity will have no effect on $d E / d x$. In this case, our treatment is equivalent to the simpler treatment of ref. [23]. In the case of the radiative energy loss rate in (2.2), or for the strongly coupled rate of energy loss (2.1) that we employ in our hybrid model, we expect that including the effects of fluid flow on the energy degradation of jets will be particularly important for jets at large rapidity.

As a simple but illustrative example, let us consider the energy lost by an energetic parton propagating through a fluid that is experiencing Bjorken flow, namely boost-invariant longitudinal expansion with no transverse flow. If the parton has a large rapidity, the fluid that it is propagating through has a large longitudinal velocity meaning that there is a substantial boost between the local fluid rest frame and the collision center-of-mass frame. Assuming that both the parton and the boost invariant fluid are produced at the same time, the longitudinal velocity of the parton coincides with the fluid velocity at its location. If the parton travels a distance $L$ in the collision center-of-mass frame during a time $t$, then according to eq. (2.7) the distance that the parton travels through the fluid in the local fluid rest frame is

$$
x_{F}(t)=\int_{0}^{t}\left|\mathbf{w}_{T}\right| d t=\frac{L}{\cosh y},
$$

where $\mathbf{w}_{T}$ and $\mathrm{y}$ are the transverse velocity of the parton its rapidity respectively. For particles with significant rapidity $y$, say $y>1$, we see that the distance they travel in the local fluid rest frame is substantially less than the distance they travel in the collision center-of-mass frame. If $d E / d x$ grows with $x$, not taking this Lorentz contraction effect into account, as in ref. [23], results in an overestimate of the amount of energy the parton loses. Conversely, incorporating this effect, as we do in this paper, will reduce the energy loss of partons with significant rapidity relative to that in ref. [23]. If the rate of energy loss is given by the expression in (2.2) inspired by radiative energy loss, this is the principal effect of flow on the energy loss suffered by an energetic parton. If the rate of energy loss is given by the strongly coupled form (2.1), as in our hybrid model, this effect is important but it is also important to note that $E_{\text {in }}$ is also different in different frames. Using (2.6),

$$
E_{\text {in }}^{F}=\frac{E_{\text {in }}}{\cosh y}
$$

in this simple Bjorken flow. The effect of this diminution in $E_{\text {in }}^{F}$ on the rate of energy loss (2.1) is complex. On the one hand, reducing $E_{\text {in }}^{F}$ reduces the rate of energy loss. On the other hand, it reduces $x_{\text {stop }}$ which increases the rate of energy loss. The net effect is not clear a priori. In the next section, we will address this issue by performing a complete simulation of jets in plasma and compare it to the results we obtained in our previous publication [23], where we took into account the changing temperature along the parton trajectory but left out the effects of the fluid velocity. 


\subsection{The effects of flow on single-jet and dijet observables}

We have re-analyzed the single jet and dijet observables studied in our previous publication [23] to include flow effects as prescribed by eq. (2.5). As already mentioned, the effect of flow for the different energy loss models we have studied is different, so we have done this reanalysis for all three expressions for the parton energy loss in (2.1) and (2.2).

Following the same procedure as in ref. [23], we embedded dijets generated by PYтнIA 8.183 [86] into boost-invariant hydrodynamic simulations of the collision dynamics. Initially, as in [23], we employed the ideal hydrodynamic simulations of $\mathrm{Pb}-\mathrm{Pb}$ collisions with center-of-mass energy $\sqrt{s}=2.76 \mathrm{AGeV}$ computed by Hirano, Huovinen and Nara [89]. These HHN simulations incorporate an equation of state obtained from the lattice calculations of ref. [102]. This equation of state has a crossover transition in a range of temperatures $180<T_{c}<200 \mathrm{MeV}$, somewhat higher than the crossover transition temperatures obtained from more recent lattice calculations. The value of the crossover temperature $T_{c}$ has quantitative effects on our results because in our model we quench jets by applying an energy loss prescription, (2.1) or (2.2), to the partons in a shower only as long as those partons are at points in spacetime where the medium has a temperature greater than $T_{c}$. When the medium temperature experienced by a given shower parton drops below $T_{c}$, we turn energy loss off. We make this sharp distinction for simplicity; it is of course unrealistic. We expect that using expressions derived for QGP like (2.1) or (2.2) overestimates the energy loss at temperatures just above $T_{c}$, since the rate of energy loss in a hadron gas is less than that in QGP meaning that as the crossover temperature is approached from above the rate of energy loss must drop in a way not captured by (2.1) or (2.2). Of course, turning the energy loss off completely is certainly an underestimate at temperatures just below $T_{c}$. If we turn energy loss off at a specified $T_{c}$ as we do, varying the value of $T_{c}$ gives us a way to estimate the systematic errors introduced into our model results by our crude treatment of late-time energy loss.

In ref. [23] we presented all our results with both $T_{c}=180 \mathrm{MeV}$ and $T_{c}=200 \mathrm{MeV}$. We do the same in the first two columns of table 1 . The first column repeats results from ref. [23] that include only the effects of variations in $T$ with no effects of the flow velocity on the energy loss included. The second column comes from our present calculations, including the effects of flow as in eq. (2.5). In each cell in the table, we quote a range of values for the parameter $\kappa_{\mathrm{sc}}$ or $\kappa_{\mathrm{rad}}$ or $\kappa_{\text {coll }}$ in the expression for the rate of energy loss $d E / d x$ of the partons in the shower that we select, (2.1) or (2.2). The range corresponds to fitting $R_{\mathrm{AA}}$ for jets with $100 \mathrm{GeV}<p_{\mathrm{T}}<110 \mathrm{GeV}$ to the upper and lower limits of the experimental uncertainty on the measured value of this quantity. We then take the entire range spanned by varying $R_{\mathrm{AA}}$ for jets with $100 \mathrm{GeV}<p_{\mathrm{T}}<110 \mathrm{GeV}$ and $-2<\eta<2$ over its experimentally allowed range [103] and by varying $T_{c}$ from 180 to $200 \mathrm{MeV}$ as the allowed range for a given $\kappa$. As described in full in ref. [23] and reviewed in appendix B, we then compute a variety of single jet and dijet observables over wide ranges in $p_{\mathrm{T}}$ and centrality and obtain good descriptions of the data, in particular using our hybrid model with $d E / d x$ given by (2.1). This does not change when we incorporate the effects of flow; what changes are the fitted values of the $\kappa$ parameters, as shown in the first two columns 


\begin{tabular}{|c||c|c||c|c||c|c|}
\hline \multirow{2}{*}{ Parameter } & \multicolumn{2}{c||}{$\begin{array}{c}\text { HHN hydro } \\
\text { without flow effects }\end{array}$} & \multicolumn{2}{c||}{$\begin{array}{c}\text { HHN hydro } \\
\text { with flow effects }\end{array}$} & \multicolumn{2}{c|}{$\begin{array}{c}\text { SH Hydro } \\
\text { with flow effects }\end{array}$} \\
\cline { 2 - 7 } & \multicolumn{2}{|c||}{$T_{c}$ range } & \multicolumn{2}{c||}{$T_{c}$ range } & \multicolumn{2}{|c|}{$T_{c}$ range } \\
& $180 \mathrm{MeV}$ & $200 \mathrm{MeV}$ & $180 \mathrm{MeV}$ & $200 \mathrm{MeV}$ & $145 \mathrm{MeV}$ & $170 \mathrm{MeV}$ \\
\hline$\kappa_{\mathrm{sc}}$ & $0.26-0.31$ & $0.30-0.35$ & $0.39-0.46$ & $0.45-0.53$ & $0.32-0.37$ & $0.35-0.41$ \\
\hline$\kappa_{\text {rad }}$ & $0.81-1.2$ & $1.0-1.6$ & $1.6-2.4$ & $2.1-3.3$ & $0.97-1.5$ & $1.2-1.8$ \\
\hline$\kappa_{\text {coll }}$ & $2.5-3.5$ & $2.9-4.2$ & $2.5-3.5$ & $2.9-4.2$ & $1.8-2.6$ & $2.2-3.0$ \\
\hline
\end{tabular}

Table 1. Values of the fit parameters needed in the specification of $d E / d x$ in our three different energy loss models, eqs. (2.1) and (2.2). The parameters are extracted by comparing model predictions for $R_{\mathrm{AA}}$ for jets with $100 \mathrm{GeV}<p_{\mathrm{T}}<110 \mathrm{GeV}$ in central $\mathrm{Pb}-\mathrm{Pb}$ at $\sqrt{s}=2.76 \mathrm{ATeV}$ collisions at the LHC to experimental data [103]. The parameters are extracted by employing three different treatments of the bulk hydrodynamic fluid. In the first two columns, we use hydrodynamic simulations from ref. [89], that we denote by HHN. The first column repeats results from ref. [23]; the second column shows the effects of including the effects of the flow velocity on the rate of energy loss as described in section 2.2. The different choices of $T_{c}$ denote the temperature below which we turn off parton energy loss in our model. In each cell in the table, the range of the fit parameter is determined by fitting to one experimentally measured $R_{\mathrm{AA}}$ data point, as described in the text. In the third column, we use hydrodynamic simulations from ref. [87] as described in the text that were provided to us by Shen and Heinz $(\mathrm{SH})$, with choices of $T_{c}$ that span the range of crossover temperatures favored by current lattice calculations.

of table 1 . Note that $\kappa_{\text {coll }}$ does not change since, as we discussed in the previous section, flow does not affect $d E / d x$ if $d E / d x$ is given by the collisional expression from (2.2). Both $\kappa_{\mathrm{sc}}$ and $\kappa_{\text {rad }}$ increase: in these cases, incorporating the effects of flow reduces the energy loss of partons with substantial rapidity and since we are fitting to $R_{\mathrm{AA}}$ for jets with pseudorapidities over the range $-2<\eta<2$ we have to increase $\kappa_{\text {sc }}$ and $\kappa_{\text {rad }}$.

Throughout the rest of this paper, we will embed jets and implement our hybrid model for their quenching in a new set of hydrodynamic simulations of $\mathrm{Pb}-\mathrm{Pb}$ collisions at $\sqrt{s}=2.76 \mathrm{ATeV}$ and $5.02 \mathrm{ATeV}$ provided to us by Shen and Heinz, based upon the codes developed by them and their collaborators in refs. [87] and [88]. These 2+1-dimensional simulations, with boost-invariant longitudinal expansion, incorporate an equation of state from ref. [104] (referred to there as the s95p-v1-PCE150 equation of state) that incorporates results from lattice QCD calculations and from a hadron resonance gas at low temperatures. With this equation of state, it is possible for us to set the $T_{c}$ below which we stop parton energy loss within a range $145 \mathrm{MeV}<T_{c}<170 \mathrm{MeV}$ that reflects results for the range of crossover temperatures from current lattice calculations, see for example refs. [105, 106]. For simplicity, the hydrodynamic calculations employ a temperatureindependent $\eta / s=1 /(4 \pi)$. Also for simplicity, we employed ensemble-averaged MCGlauber initial conditions [107], neglecting all effects of event-by-event fluctuations. Because we are not concerned with the evolution below $T_{c}$, we evolved the initial conditions using viscous hydrodynamics alone, with no cascade afterburner.

As shown in the third column of table 1 , we have refitted the parameters $\kappa_{\mathrm{sc}}, \kappa_{\mathrm{rad}}$ and $\kappa_{\text {coll }}$ in the expressions (2.1) and (2.2) to the experimentally measured $R_{\mathrm{AA}}$ for jets 
with $-2<\eta<2$ and $100 \mathrm{GeV}<p_{\mathrm{T}}<110 \mathrm{GeV}$ using the SH hydro simulations and setting $T_{c}$ to $145 \mathrm{MeV}$ or $170 \mathrm{MeV}$ to yield some sense of the theoretical systematic uncertainty as before. The values of the parameters so obtained are smaller than those in the second column, reflecting the lower value of $T_{c}$ meaning that energy loss continues for the partons in the showers for a longer time. We have checked that most of the difference between the parameter values in the second and third columns is indeed due to the change in the value of $T_{c}$, as anticipated. Perhaps coincidentally, the parameter values in the third column are quite similar to those from ref. [23] given in the first column: the consequences of adding flow effects and lowering $T_{c}$ to a large degree cancel.

In appendix $\mathrm{B}$, we update the results of ref. [23] for the jet $R_{\mathrm{AA}}$ as a function of $p_{\mathrm{T}}$ and centrality, and for the dijet imbalance in $\mathrm{Pb}-\mathrm{Pb}$ collisions at $\sqrt{s}=2.76 \mathrm{ATeV}$, comparing them to CMS data $[6,103]$. We see that with SH hydro and a lower $T_{c}$, just as with HHN hydro and a higher $T_{c}$ in ref. [23], when we fit the one parameter $\kappa_{\mathrm{sc}}$ in our hybrid model to one data point the model does a very good job of describing these full data sets. In appendix $\mathrm{C}$, we present results for $R_{\mathrm{AA}}$ and the dijet imbalance for $\mathrm{Pb}-\mathrm{Pb}$ collisions with $\sqrt{s}=$ $5.02 \mathrm{ATeV}$. These constitute predictions for the LHC heavy ion run coming late this year.

The improvements we have introduced in our description of the dynamics of the expanding droplet of QGP in going from the first column of table 1 to the second column to the third column have resulted in changes to the numerical values of $\kappa_{\mathrm{sc}}, \kappa_{\text {rad }}$ and $\kappa_{\text {coll }}$. However, the conclusions about the implications of the values of these parameters that we reached in ref. [23] all remain unchanged. The extracted values of $\kappa_{\text {rad }}$ and $\kappa_{\text {coll }}$ imply such large "weak" couplings or such large logarithmic corrections to the leading order energy loss result that a perturbative analysis is called into question. ${ }^{4}$ The extracted value of $\kappa_{\mathrm{sc}}$ in the strongly coupled expression (2.1) for $d E / d x$ in our hybrid model corresponds to a stopping distance for energetic partons in the strongly coupled QGP of QCD that is about three to four times longer than that in the strongly coupled plasma of $\mathcal{N}=4 \mathrm{SYM}$ theory. And, as can be seen by comparing the plots in appendix B to those in ref. [23], the hybrid model with the improvements that we have introduced continues to provide a good description of data on both single jet and dijet observables over a wide range of jet $p_{\mathrm{T}}$ and event centrality.

\subsection{Species dependence of jet suppression}

Although the central conclusions we draw from confronting our hybrid model with the measured observables compiled in appendix B are unchanged from those we reached in ref. [23], the conclusions that we drew there about the species dependence of jet suppression are substantially affected by the inclusion of the effects of flow on parton energy loss that we have described in the preceding sections. In the left panel of figure 1, we repeat a calculation from ref. [23], now with $\mathrm{SH}$ hydro and the lower $T_{c}$ range from the third column of table 1 , and reach different conclusions. We show the ratio of the number of quark-initiated jets to the number of gluon-initiated jets with jet momenta in the range $100<p_{\mathrm{T}}<150 \mathrm{GeV}$ and $|\eta|<2$ as a function of centrality for the three models we have studied. As in [23],

\footnotetext{
${ }^{4}$ For an analysis of the effects of large logarithmic corrections to medium parameters, see refs. [108, 109].
} 

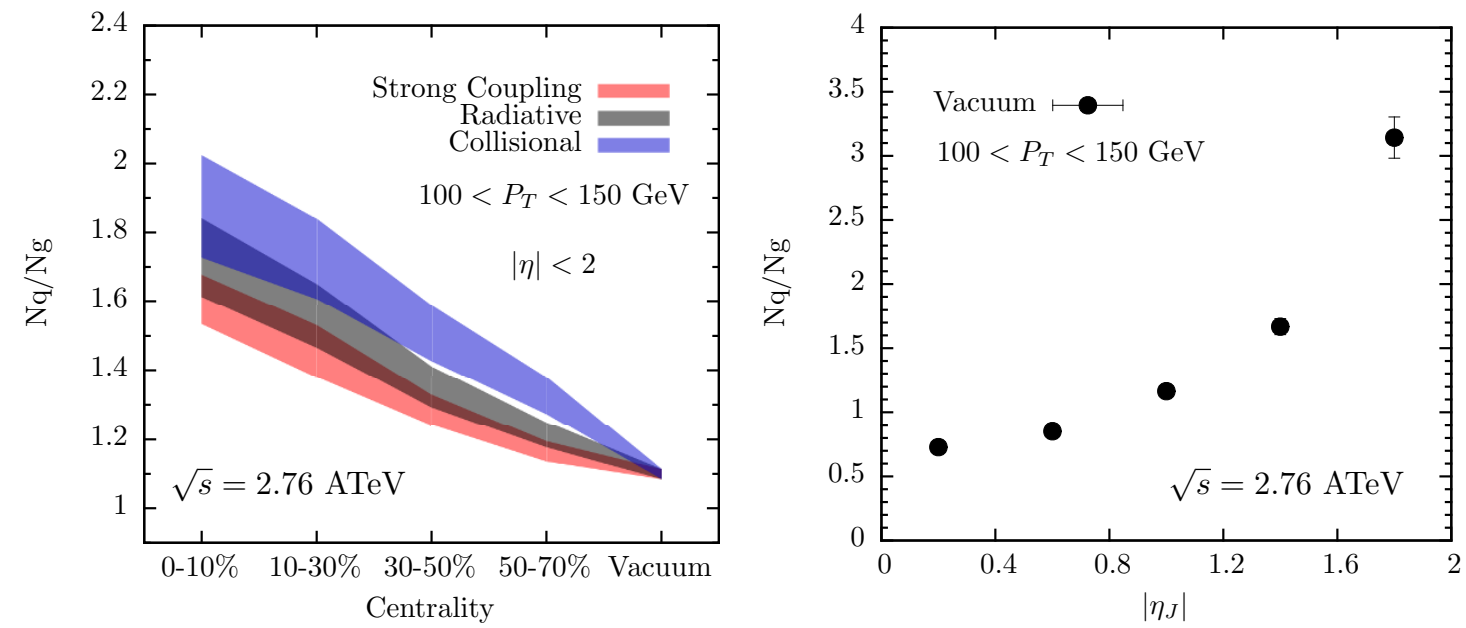

Figure 1. Left panel: ratio of quark-initiated jets to gluon-initiated jets with $|\eta|<2$ as a function of centrality for different models. Right panel: ratio of quark-initiated jets to gluon-initiated jets as a function of jet pseudorapidity for p-p collisions in vacuum, according to PYTHIA.

we have defined the species of the jet-initiator from our PүтніA "data" (in a way that is impossible to do in experimental data) as the identity of the parton that initiated the DGLAP shower to which the hardest particle in the jet belongs. The inclusion of the effects of flow on parton energy loss has resulted in the bands corresponding to our three different models being much closer to each other in figure 1 than they were in ref. [23]. The power to discriminate between models of energy loss implicit in figure 1 is much less than was the case in ref. [23]. This change arises as a combination of two facts. First, as we discussed in section 2.2 the inclusion of the effects of flow reduces the energy loss of partons (both quarks and gluons) at large rapidity if $d E / d x$ is given by the strong coupling expression (2.1) or the radiative expression from (2.2), but leaves things unchanged if the collisional expression from (2.2) is employed. Second, as shown in the right panel of figure 1, without any energy loss there are many more quark-initiated jets at large rapidity than at mid-rapidity for jets with the same range of $p_{\mathrm{T}}$ as in the left panel of the figure. As the pseudorapidity increases, jets in this interval of transverse momentum become more and more quark dominated. The consequences of these two facts in concert is that the many quark-initiated jets at large rapidity lose less energy once flow effects are included than they did in the analysis of ref. [23], if $d E / d x$ depends on flow as in either the strongly coupled or radiative expressions. In these cases, the effects of flow on the rate of energy loss serve to enhance the relative weight of the large rapidity jets in the final jet sample, and these large rapidity jets are predominantly quark-initiated. This means that, after summing over $|\eta|<2$ as in the left panel of figure 1, the inclusion of the effects of flow pushes the strongly coupled and radiative $N_{q} / N_{g}$ bands in this figure upwards, bringing both much closer to the collisional band. This happens even though, as we mentioned in section 2.1 and discussed in detail in ref. [23], the Casimir-dependence of the rate of energy loss in (2.1) is weaker than in (2.2): this difference in the Casimir dependence of $d E / d x$ pushes the strongly coupled $N_{q} / N_{g}$ band in the left panel of figure 1 downward relative 

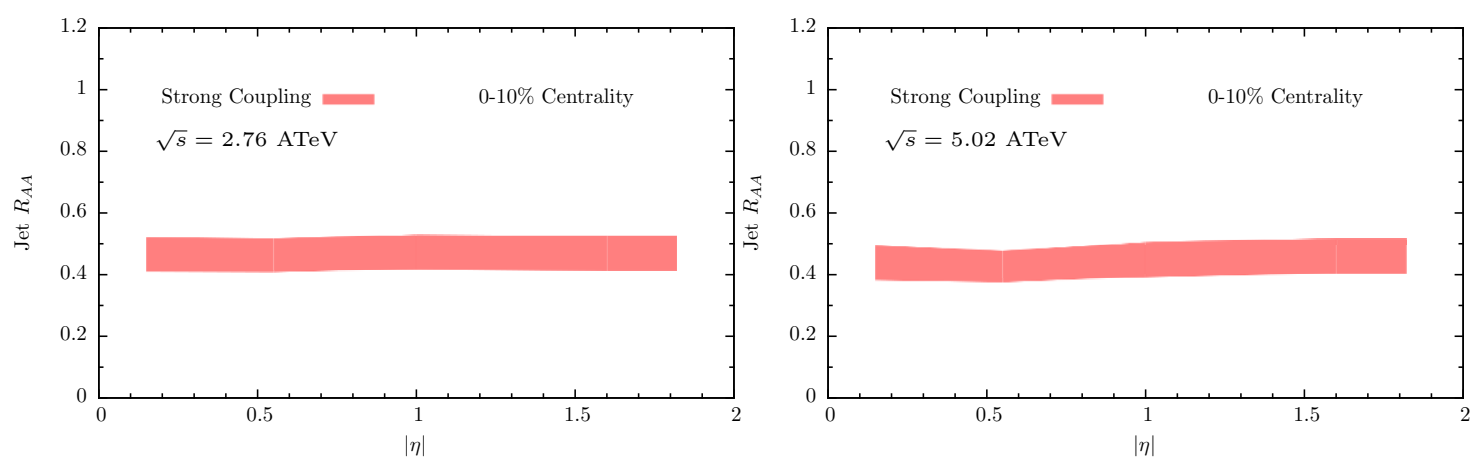

Figure 2. Pseudorapidity dependence of jet $R_{A A}$ in our hybrid model for $\sqrt{s}=2.76 \mathrm{ATeV}$ (left) and $\sqrt{s}=5.02 \mathrm{ATeV}$ (right).

to the collisional band, but the effects of flow in concert with the rapidity-dependence of the production of quark and gluon jets in p-p collisions brings it back up. Consequently, our current results render the species-dependence of quenching rather insensitive to the underlying energy loss dynamics.

We close this section by noting that despite the substantial rapidity dependence of the species-dependence of jet production, and of jet quenching, the resulting net jet $R_{\mathrm{AA}}$ is remarkably independent of rapidity in our hybrid model, as shown in figure 2. This was noted previously in ref. [110], and we find the same behavior in our control models in which we choose the dependence of $d E / d x$ according to either one of the expressions (2.2). Although given the strong species-dependence manifest in the right panel of figure 1 the flatness of the rapidity dependence seen in figure 2 appears coincidental, it is in agreement with experimental results from the ATLAS collaboration [17].

\section{Boson-jet correlations, including predictions for $\sqrt{s}=5.02 \mathrm{ATeV}$ col- lisions and for Z-jet correlations}

The extensive exploration in ref. [23] demonstrated that the hybrid model that we have developed describes currently available inclusive jet and dijet data from LHC heavy ion collisions rather successfully. In appendix B, we compile updated versions of these results, using SH hydro and taking flow effects into account as we described in the previous section, and we reconfirm the conclusions of ref. [23]. This gives us considerable confidence in the model framework. However, this class of observables proves not to be very sensitive to whether we choose $d E / d x$ as in (2.1) or (2.2) [23]. The distinctions between the strongly coupled form for $d E / d x$ and our two control models provided by these observables are not sufficient to differentiate between these different hypotheses for the microscopic dynamics of energy loss, at least with present uncertainties. The strongly coupled form for $d E / d x$ does provide a better description of the dijet imbalance, but its predictions are not sufficiently distinct from those of the control models. We must, therefore, consider further observables.

In deciding how to go further, we face a choice. We could start adding more physics to the model, which would allow us to confront new classes of observables. For example, if 
we were to add in the transverse momentum kicks that the medium delivers to the shower partons passing through it, and the recoil that the shower partons delivers to the medium, we could engage with jet shape observables. This is an attractive prospect, but we defer it to future work. Before adding to the model, and in particular before adding a second free parameter, it is our responsibility to first ask whether there are further observables that our present model, with its single parameter already fixed as described in the previous section, could reasonably be expected to describe. This is our goal in this paper.

For most of the remainder of this paper, we turn to observables involving jets back-toback with either an energetic and isolated photon or a Z-boson. We will keep the parameter $\kappa_{\mathrm{sc}}$ in our hybrid model fixed exactly as in the previous section, and confront our model with existing data on three different observables describing $\gamma$-jet correlations in $\mathrm{Pb}-\mathrm{Pb}$ collisions at $\sqrt{s}=2.76 \mathrm{ATeV}$. As before, our model describes the data well, although it is fair to note in advance that the statistical error bars on these measurements are substantial because to this point the data sets of $\gamma$-jet events have not been large. Nevertheless, the confrontation of the hybrid model with these three new sets of data provides a strong independent validation of our hybrid model, which has no further adjustable parameters.

Our central purpose in this section is to use our hybrid model to provide predictions for the measurements that the data sets with higher statistics by about an order of magnitude that are anticipated late this year will make possible. To this end, we provide predictions for three $\gamma$-jet observables and three Z-jet observables in $\mathrm{Pb}-\mathrm{Pb}$ collisions at $\sqrt{s}=5.02 \mathrm{ATeV}$. In making these predictions, we will keep the parameter $\kappa_{\mathrm{sc}}$ in the hybrid model and the parameters $\kappa_{\text {rad }}$ and $\kappa_{\text {coll }}$ in the control models set to the same values that we obtained in the previous section by fitting to one data point at $\sqrt{s}=2.76 \mathrm{ATeV}$. The principal change in going from $\sqrt{s}=2.76 \mathrm{ATeV}$ to $\sqrt{s}=5.02 \mathrm{ATeV}$ is a (modest) increase in the temperature of the plasma. The principal effects of this increase arise from the explicit temperature dependence in (2.1) or (2.2), and so are included in our analysis. We are leaving out any small decrease in the values of the $\kappa$ 's that may arise if the plasma becomes slightly less strongly coupled, anticipating that this effect will likely be too small to be resolved given other uncertainties, both theoretical and experimental.

Although the observables that we study in this section are similar in some respects to those that describe dijet events, since neither $\gamma$ 's nor Z-bosons interact strongly with the plasma, when one triggers on an energetic $\gamma$ or Z-boson the jets produced in the same hard scattering have points of origin that are distributed through the collision volume differently than is the case for dijet pairs, where selection bias favors points of origin closer to the surface where at least one of the jets will suffer less energy loss. This means that the jets produced in association with a $\gamma$ or a Z-boson sample a different path-length distribution than the jets in dijet pairs. A second difference between boson-jet events and dijet events is that both $\gamma$ 's and Z-bosons are much more likely to be produced in association with a quark jet than with a gluon jet. The third difference is that since the $\gamma$ or Z-boson suffers no energy loss, as they do not interact with the medium, their energy and direction are good proxies for the initial energy and initial direction of the hard parton (usually, the quark) going in the opposite direction. This is quite different than in the case of dijet events, where generically both jets should be expected to have lost some energy. Of course, 
the hard parton opposite a $\gamma$ or Z-boson may split into more than one jet; in the analysis of $\gamma$-jet and Z-jet correlations, it cannot be assumed that the boson is back-to-back with only one jet in the final state.

\subsection{Generation and selection of Monte Carlo events}

For our study of photon-jet correlations we analyzed $10^{5}$ hard scattering processes, in Pythia 8.183 [86] p-p collisions at $\sqrt{s}=2.76 \mathrm{TeV}$ and another $10^{5}$ such events in PythiA pp collisions at $\sqrt{s}=5.02 \mathrm{TeV}$. We require at least one photon with a transverse momentum above a desired cut, typically choosing $p_{\mathrm{T}}{ }^{\gamma}>p_{\mathrm{T}}{ }^{\text {cut }}=60 \mathrm{GeV}$, and with a pseudorapidity in the range $\left|\eta_{\gamma}\right|<1.44$. We set the ${p_{\mathrm{T}}}^{\text {min }}$ parameter in PYTHIA (basically, the minimum momentum transfer in the hard processes that PYTHIA is sampling) safely lower than $p_{\mathrm{T}}{ }^{\text {cut }}$, for example choosing $p_{\mathrm{T}}{ }^{\mathrm{min}}=40 \mathrm{GeV}$ if we are recording events that include a photon with $p_{\mathrm{T}}{ }^{\gamma}>60 \mathrm{GeV}$, since PYтнIA does not reliably reproduce the photon spectrum all the way down to its $p_{\mathrm{T}}^{\min }{ }^{5}$ We placed each of these $10^{5}$ events at random in the transverse plane of a $\mathrm{Pb}-\mathrm{Pb}$ collision, with $\sqrt{s}=2.76 \mathrm{ATeV}$ or $5.02 \mathrm{ATeV}$, choosing the location according to the probability distribution for the number of binary collisions occurring at each transverse position according to an optical Glauber model. We keep the direction of the photon (in azimuth and in rapidity) as it was in the PүтніA p-p collision from which we have taken it. We generate the hard processes in PүтніA without underlying p-p events because we embed each hard process into a hydrodynamic description of the matter produced in the heavy ion collision, meaning that including the underlying p-p event from PYTHIA would be double-counting. As we described in the previous section, we use the boost-invariant 2+1-dimensional hydrodynamical simulations of the expanding cooling droplet of matter produced in the heavy ion collision from ref. [87] to determine the local temperature and local velocity vector of the fluid at the position of each of the partons in the jet shower described by PYTHIA, as they propagate through the cooling hydrodynamic fluid as a function of time. We follow each parton in the shower, and as long as a parton sees a local temperature that is greater than $T_{c}$ we reduce its energy at a rate $d E / d x$ given by (2.1) in our hybrid model, or by (2.2) if we are investigating one of our two control models. When a parton that has lost some energy branches, we start each of its daughters off with the same fractional energy loss as the parent had when it branched. The daughters then lose further energy according to (2.1) in our hybrid model or (2.2) in our control models. In this way, we compute the energy lost by each of the partons in the showers produced by the hard scattering process, in so doing modifying the partons in the final state relative to what they were in the original PyтнiA event. As in ref. [23], we neglect energy loss in the pre-equilibrium stage of the collision before the hydrodynamic evolution of ref. [87] starts at $\tau=0.6 \mathrm{fm}$. We have described these procedures in greater detail in ref. [23]. As in the previous section, we vary our choice of the temperature $T_{c}$ below which we turn off the energy loss over the range $145<T_{c}<170 \mathrm{MeV}$. The result of our analysis is $10^{5}$ hard scattering events (for each collision energy and for each choice of $T_{c}$ ) in which the

\footnotetext{
${ }^{5}$ For one of our observables, we wanted to include photons with $p_{\mathrm{T}}{ }^{\gamma}>p_{\mathrm{T}}{ }^{\text {cut }}=40 \mathrm{GeV}$ in our analysis. For this observable, we ran $10^{5}$ events with $p_{\mathrm{T}}{ }^{\mathrm{min}}=20 \mathrm{GeV}$.
} 
partons accompanying the photon have lost energy as a result of their passage through the hydrodynamic medium.

Next, we analyze the $10^{5}$ events in order to "measure" each observable that is of interest to us. In doing so, we only analyze the (modified) parton showers coming from the hard scattering. We do not include any of the particles that would be created as the hydrodynamic fluid freezes out. This means that we do not have any background particles and we therefore do not perform any background subtraction in our calculation of observables. When we compare to data, we of course compare to measurements made after the experimentalists have done their background subtraction. For this reason, for those observables which have not been fully unfolded in the experimental analysis we include a smearing procedure, to be describe in more detail later, to mimic resolution effects.

Although we of course know (from PYTHIA) exactly which photons in our events are the photons coming from the originating hard process (prompt photons) and which photons are instead produced in the parton shower (fragmentation photons), we do not use this information in our computation of observables. Instead, we perform an isolation analysis of all high energy photons in our Monte Carlo data, patterned upon what experimentalists must do. This procedure allows us to study a sample of photons which is closer to that in experimental analyses, since in experiments prompt and fragmentation photons can only be distinguished via isolation cuts, with finite efficiencies. As it turns out, quenched jets associated with prompt photons constitute about $80 \%$ of our final "data" sets, with about $20 \%$ coming from fragmentation of jets in events without a prompt photon.

For readers who would like a little more detail, our procedure is as follows. We select events containing at least one photon with $p_{\mathrm{T}}^{\gamma}>p_{\mathrm{T}}{ }^{\text {cut }}$ and $\left|\eta_{\gamma}\right|<1.44$. After quenching the parton showers in these events as described above, following the analysis of refs. $[8,111]$ we consider a photon to be isolated if the sum of the energy of all the particles within a cone around this photon of radius $\Delta r \equiv \sqrt{\Delta \phi^{2}+\Delta \theta^{2}}=0.4$ is below $5 \mathrm{GeV}$. Contrary to what is done in the analysis of experimental data, we perform this isolation cut at the partonic level, instead of at the hadronic level. In the unlikely situation that two or more isolated photons are found in a single event, we treat the one with the highest transverse momentum as the leading photon. Next, we construct a sample of photon-jet pairs from our sample of isolated photons. We reconstruct jets using the anti- $k_{T}$ algorithm $[112,113]$, typically choosing $R=0.3$, and keep those events in which we find an associated jet at an angular distance $\Delta \phi>7 \pi / 8$ from the isolated photon. This angular cut suppresses the contribution of events in which the isolated photon is a fragmentation photon and events with more than one associated jet to our final photon-jet sample.

We generate a sample of Z-jet events via an analogous procedure. We select $10^{5}$ PyтнiA events in which a hard scattering process produces a Z-boson with $p_{\mathrm{T}}{ }^{Z}>p_{\mathrm{T}}{ }^{\text {cut }}$, again typically choosing $p_{\mathrm{T}}{ }^{\text {cut }}=60 \mathrm{GeV}$. We need not worry about fragmentation Z-bosons: the large mass of the $\mathrm{Z}$ makes it very unlikely that they are produced in the parton showers. We therefore need not apply any isolation procedure: any $\mathrm{Z}$ is a prompt $\mathrm{Z}$. For the sake of simplicity, we use the same $p_{\mathrm{T}}$ and $\eta$ cuts applied to photon events. We reconstruct jets using the anti- $k_{T}$ algorithm as before, again typically choosing $R=0.3$, and obtain our 
Z-jet sample by requiring an associated jet at an angular distance $\Delta \phi>7 \pi / 8$ from the Z. In experimental measurements, Z-bosons are reconstructed through their di-muon decay. Since high energy muons are not modified at all by their passage through the plasma, and since the properties of the associated jets are the same no matter how the Z-boson decays, to increase the size of our sample we simply do not allow our Z-bosons to decay, keeping all of them in our sample.

\subsection{Photon-jet observables: comparison with experimental results at $\sqrt{s}=$ $2.76 \mathrm{ATeV}$ and predictions for $\sqrt{s}=5.02 \mathrm{ATeV}$}

We use the Monte Carlo samples of photon-jet events, prepared and quenched as described above, to construct different observables which can be confronted with experimental data. In this section, we describe these observables, we show the results obtained from our model for collisions at $\sqrt{s}=2.76 \mathrm{ATeV}$ and compare them to data, and we provide the predictions that we obtain from our model for collisions at $\sqrt{s}=5.02 \mathrm{ATeV}$.

We first construct the photon-jet imbalance. This is analogous to the dijet imbalance, except that here we have a photon instead of a leading jet. In the case of dijets we define the associated jet as the jet in the pair that has less transverse momentum, meaning that the ratio of the transverse momentum of the associated jet to that of the leading jet is less than one by definition. This is not so in the case of the photon-jet imbalance, since the associated jet can have more or less transverse momentum than the photon. Defining $x_{J \gamma} \equiv p_{\mathrm{T}}{ }^{\text {jet }} / p_{\mathrm{T}}{ }^{\gamma}$, this observable can be less than or greater than one. For example, in p-p collisions in vacuum, an associated jet can have more transverse momentum than the photon if there is a second jet in the event, in the same hemisphere as the photon. In a heavy ion collision, the energy loss experienced by the partons propagating through the medium pushes $x_{J \gamma}$ downwards. However, if the passing jet sweeps particles from the plasma into the jet cone this can in principle push $x_{J \gamma}$ upwards, but this effect is expected to be small at large energies. Our hybrid model neglects this possibility, meaning that in every event in our sample $x_{J \gamma}$ is less than (or equal to) what it would have been in the absence of the medium.

In figure 3 , we show the distribution of the imbalance in the transverse momentum of the associated jet relative to that of the photon, $x_{J \gamma}$, for events with two different centralities and for events with two different collision energies. Following the conventions established in the experimental analyses in refs. [8, 111], these distributions are normalized to the total number of photons, rather than to the total number of photon-jet pairs; integrating each of the curves in figure 3 therefore yields a number below one. Also as in the experimental analysis, we only consider photons with transverse momentum $p_{\mathrm{T}}{ }^{\gamma}>60 \mathrm{GeV}$. The associated jet is reconstructed with the anti- $k_{T}$ algorithm with $R=0.3$; we only count events in which the associated jet has a transverse momentum $p_{\mathrm{T}}{ }^{\text {jet }}>30 \mathrm{GeV}$. The widths of the colored bands that illustrate our results in this figure - and in many figures that follow - incorporate both the uncertainty that comes from varying $T_{c}$ between 145 and $170 \mathrm{MeV}$ and the uncertainty that comes from varying our model parameter $\kappa_{\mathrm{sc}}$ over its allowed range $0.32<\kappa_{\mathrm{sc}}<0.41$, determined in section 2 . That is, we obtain four curves by repeating our calculation of the observable in question, here $x_{J \gamma}$, with $T_{c}$ and $\kappa_{\mathrm{sc}}$ each 

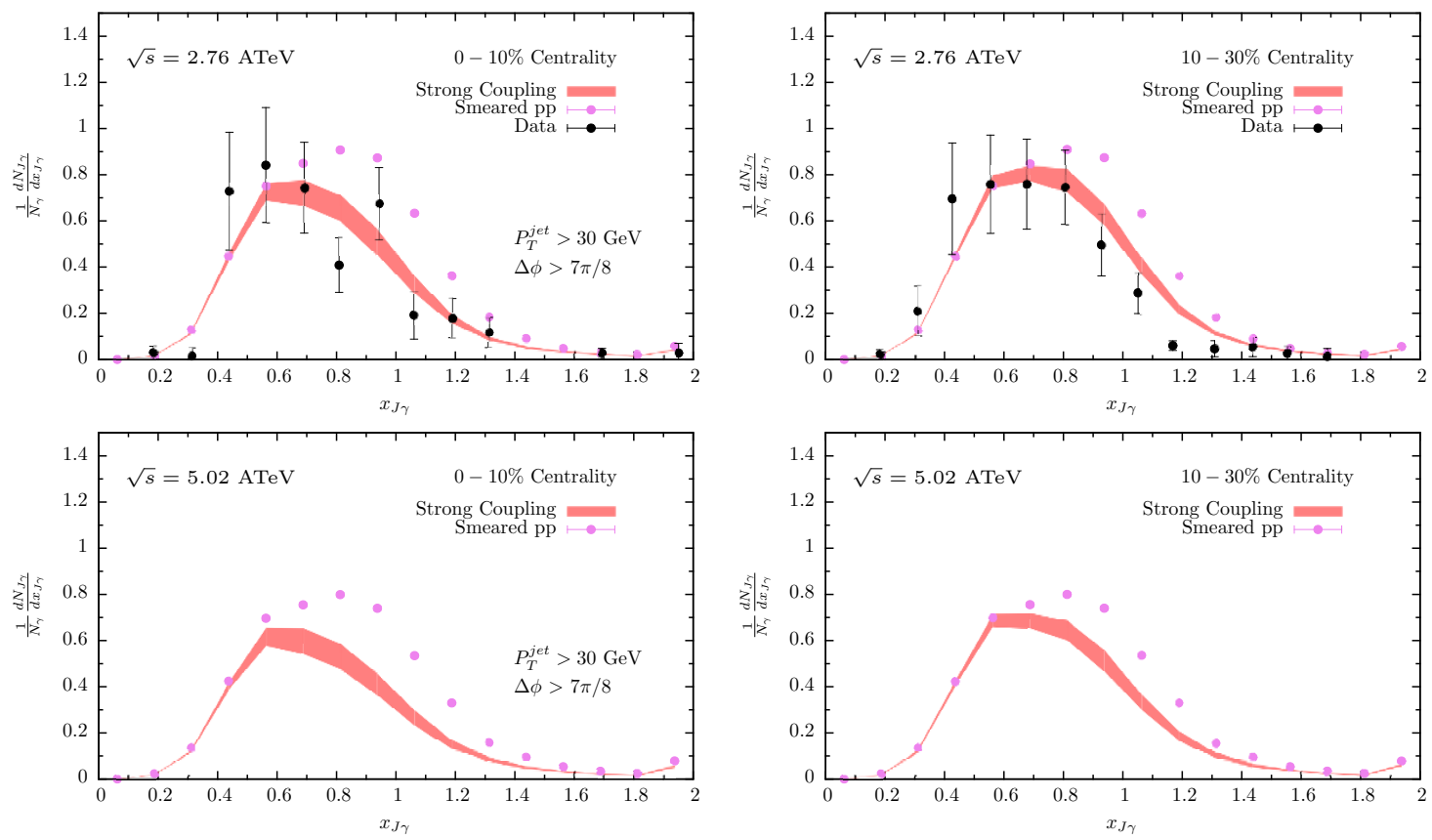

Figure 3. Distribution of the transverse momentum imbalance of photon-jet pairs, $x_{J \gamma} \equiv$ $p_{\mathrm{T}}{ }^{\text {jet }} / p_{\mathrm{T}}{ }^{\gamma}$, for $\mathrm{Pb}-\mathrm{Pb}$ collisions. The left and right panels show the $0-10 \%$ and $10-30 \%$ most central events, respectively. The upper panels show our results for collisions with $\sqrt{s}=2.76 \mathrm{ATeV}$, as well as data from ref. [111]. The lower panels show our predictions for heavy ion collisions with $\sqrt{s}=5.02 \mathrm{ATeV}$, anticipated for late this year.

set to its lowest and its highest value, and plot the band that extends from whichever one of the four curves is lowest to whichever curve is highest at each point in the figure.

As a reference, in figure 3 we display the $x_{J \gamma}$ distribution in p-p collisions at the same nucleon-nucleon energy as predicted by PYTHIA, i.e. with no medium-induced parton energy loss. In order to mimic the effects of jet-energy resolution on the transverse momentum of the associated jet obtained in the analysis of the $\mathrm{Pb}-\mathrm{Pb}$ data after the subtraction of the background, in our p-p results we have smeared the momenta of the associated jets obtained from our Monte Carlo calculation with a centrality-dependent Gaussian broadening. The parameters of this smearing, reported in ref. [114], were tuned to reproduce the measured distributions after embedding PYTHIA-generated photon-jet samples into real lead-lead events. For this reason, the smeared proton-proton distribution is not identical in the left and right panels of figure 3, which display our results for $\mathrm{Pb}-\mathrm{Pb}$ collisions with two different centralities. For the present, these smeared p-p results are the correct reference to which both the experimental results as measured by the CMS collaboration [7] and our results for quenched jets in $\mathrm{Pb}-\mathrm{Pb}$ collisions should be compared. (See ref. [115] for fully unfolded experimental results to come.) Given that in our $\mathrm{Pb}-\mathrm{Pb}$ simulations there is no background, and consequently no background subtraction effects, in obtaining the results represented by the colored bands in figure 3 we have applied the same smearing procedure to our simulated quenched jets. The smearing parameters are at present known only for $\sqrt{s}=2.76 \mathrm{ATeV}$, not for $\sqrt{s}=5.02 \mathrm{ATeV}$. For the present, we have decided to employ the 
same smearing parameters at the higher collision energy. Our predictions therefore assume that the effects of background subtraction on these observables are similar at these two collision energies.

The differences between the (smeared) proton-proton Monte-Carlo data and the results of our in-medium calculations displayed in figure 3 are due to energy loss. The sample of isolated photons we have used to construct these distributions is dominated by prompt photons, which do not lose momentum when traversing the plasma. ${ }^{6}$ The partons in the showers that become the associated jets, however, interact strongly with the medium and lose energy according to (2.1) as they propagate through it. In some cases, this pushes the transverse energy of the associated jet below $30 \mathrm{GeV}$, meaning that the event does not get counted as a photon-jet in our $\mathrm{Pb}-\mathrm{Pb}$ analysis although it was counted in our p-p analysis. This is why the integrals of the curves illustrating our $\mathrm{Pb}-\mathrm{Pb}$ results in figure 3 are smaller than the integrals of our p-p results. More on this below. In other cases, when the transverse energy of the associated jet remains above $30 \mathrm{GeV}$, the effect of the energy loss is to reduce $x_{J \gamma}$, displacing the photon-jet imbalance distribution toward smaller values of $x_{J \gamma}$.

Keeping in mind that we fixed the single parameter in our hybrid model by comparing it to the single-jet suppression $R_{A A}$, see section 2, it is remarkable how well the photon-jet imbalance distribution that we obtain from our model agrees with CMS measurements in $\sqrt{s}=2.76 \mathrm{ATeV}$ collisions in both centrality bins in figure 3 . The fact that only one side of photon-jet pair loses energy makes the interpretation of this observable cleaner than in the case of the dijet imbalance. Of course, at present the large statistical uncertainties in the photon-jet measurements is a limitation on their use to differentiate between different model assumptions for the rate of energy loss $d E / d x$, which is to say a limitation on their use as diagnostics of the mechanism of energy loss. We illustrate this point in appendix D, where we present the results that we obtain by repeating our photon-jet and Z-jet calculations using the control models for $d E / d x$ in (2.2). We find that these control models make predictions that are distinct from those of our hybrid strong/weak coupling model, with $d E / d x$ given by (2.1), but the distinctions are too small to be resolved by the present data, with their statistical uncertainties.

Given these considerations, perhaps the most important results that we can use our model to provide are our predictions for the upcoming runs of the LHC, at $\sqrt{s}=5.02 \mathrm{ATeV}$, where the photon-jet data sets are expected to be larger by about an order of magnitude and the statistical uncertainties are expected to be substantially smaller than at present. For this reason, in the lower panels of figure 3 we show the predictions of our hybrid model, with its strongly coupled form for the rate of energy loss, for the photon-jet imbalance distribution in $\mathrm{Pb}-\mathrm{Pb}$ collisions at $\sqrt{s}=5.02 \mathrm{ATeV}$. We have applied the same kinematical cuts used in current measurements (and our calculations) at $\sqrt{s}=2.76 \mathrm{ATeV}$. The imbalance distribution shows little dependence on the collision energy.

To further test the success of our hybrid approach, we now turn to exploring other photon-jet observables. In figure 4 we show the fraction of isolated photons that come

\footnotetext{
${ }^{6}$ The small fraction of fragmentation photons which fulfill the isolation requirement do suffer energy loss via the quenching of the partons from which they originate. This effect is small for this observable, but it can have consequences for more differential observables, as we will discuss below.
} 

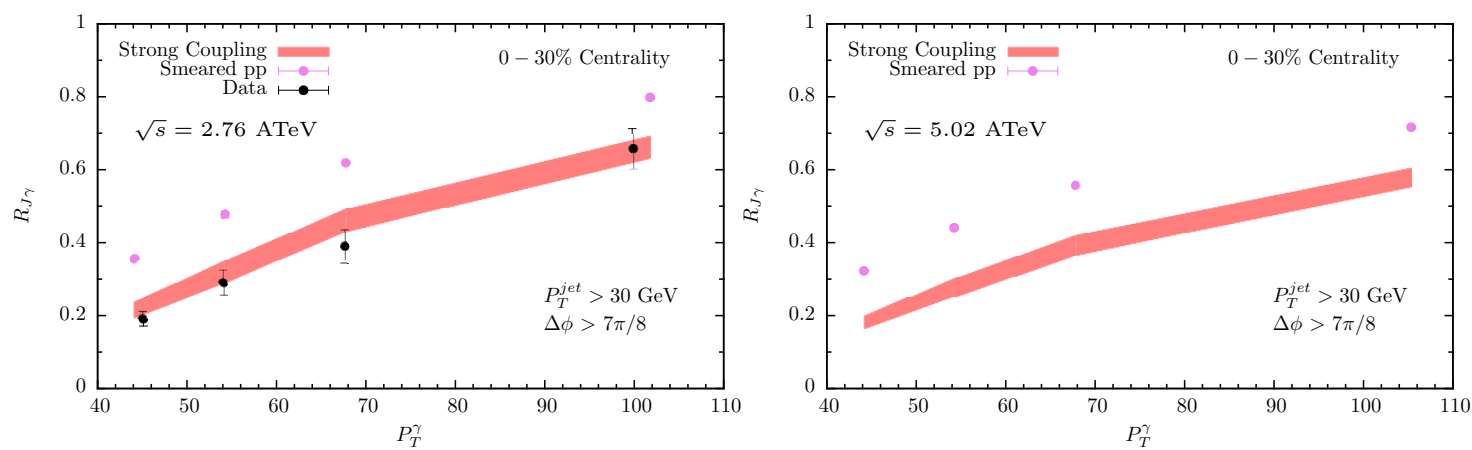

Figure 4. Fraction of events with an isolated photon in which we find a photon-jet pair, which is to say in which we find an associated jet with $p_{\mathrm{T}}{ }^{\text {jet }}>30 \mathrm{GeV}$ at an azimuthal angle more than $7 \pi / 8$ away from that of the isolated photon. We plot this fraction as a function of the photon transverse momentum, in collisions with $\sqrt{s}=2.76 \mathrm{ATeV}$ (left) and $\sqrt{s}=5.02 \mathrm{ATeV}$ (right). The colored band shows the results from our hybrid model, with its strongly coupled form for the rate of energy loss. For comparison, the violet dots show the smeared p-p calculations (see text for details). Our results for $\sqrt{s}=2.76 \mathrm{ATeV}$ are compared to CMS data [111].

with an associated jet, as reconstructed with the anti- $k_{T}$ algorithm with $R=0.3$, that has $p_{\mathrm{T}}{ }^{\text {jet }}>30 \mathrm{GeV}$ and $\Delta \phi>7 \pi / 8$. We plot this quantity, which we denote by $R_{J \gamma}$, as a function of the transverse momentum of the isolated photon for heavy ion collisions with $\sqrt{s}=2.76 \mathrm{ATeV}$ (left) and $\sqrt{s}=5.02 \mathrm{ATeV}$ (right). In the plots in figure 4 , the smeared proton-proton PYTHIA simulations are represented by the violet dots, the results of our hybrid strong/weak coupling model are represented by the colored band, and the experimental results from ref. [111] for $\sqrt{s}=2.76 \mathrm{ATeV}$ are the black data points. ${ }^{7}$ The broad $x_{J \gamma}$ distribution seen in figure 3 implies that this ratio must be an increasing function of $p_{\mathrm{T}}^{\gamma}$ for both collision energies, since the $30 \mathrm{GeV}$ cut on the associated jet energy is more and more easily satisfied as the momentum of the photon is made larger and larger. Quenching reduces $R_{J \gamma}$ since it pushes the energy of some of the associated jets below $30 \mathrm{GeV}$. As for the photon-jet imbalance distribution, we find good agreement between the $R_{J \gamma}$ obtained from our hybrid model and that measured in present experiments with $\sqrt{s}=2.76 \mathrm{ATeV}$, in this case for all values of the photon momenta. ${ }^{8}$ Our predictions for heavy ion collisions with $\sqrt{s}=5.02 \mathrm{ATeV}$ are shown in the right panel of figure 4 . As before, this observable shows little sensitivity to the collision energy, at least within these kinematical cuts.

We have also analyzed the spectrum of jets produced in association with an isolated photon. In figure 5 , we show the ratio of the spectrum of associated jets in $\mathrm{Pb}-\mathrm{Pb}$ collisions to that in proton-proton collisions, $I_{A A}$, for two different ranges of photon transverse

\footnotetext{
${ }^{7}$ Note that the ratio of the integral of the photon-jet imbalance in $\mathrm{Pb}-\mathrm{Pb}$ collisions to that in $\mathrm{p}-\mathrm{p}$ collisions, see figure 3 , is the ratio of $R_{J \gamma}$ for all photons with $p_{\mathrm{T}}^{\gamma}>60 \mathrm{GeV}$ in $\mathrm{Pb}-\mathrm{Pb}$ collisions to that in p-p collisions. The fact that the colored band lies below the violet dots in figure 4 was therefore foreshadowed in figure 3 .

${ }^{8}$ Note that in order to extend our calculations of $R_{J \gamma}$ down to $40 \mathrm{GeV}<p_{\mathrm{T}}{ }^{\gamma}<50 \mathrm{GeV}$ we used a sample of Monte Carlo events with the PYтнiA parameter $p_{\mathrm{T}}{ }^{\text {min }}$ set to $20 \mathrm{GeV}$.
} 

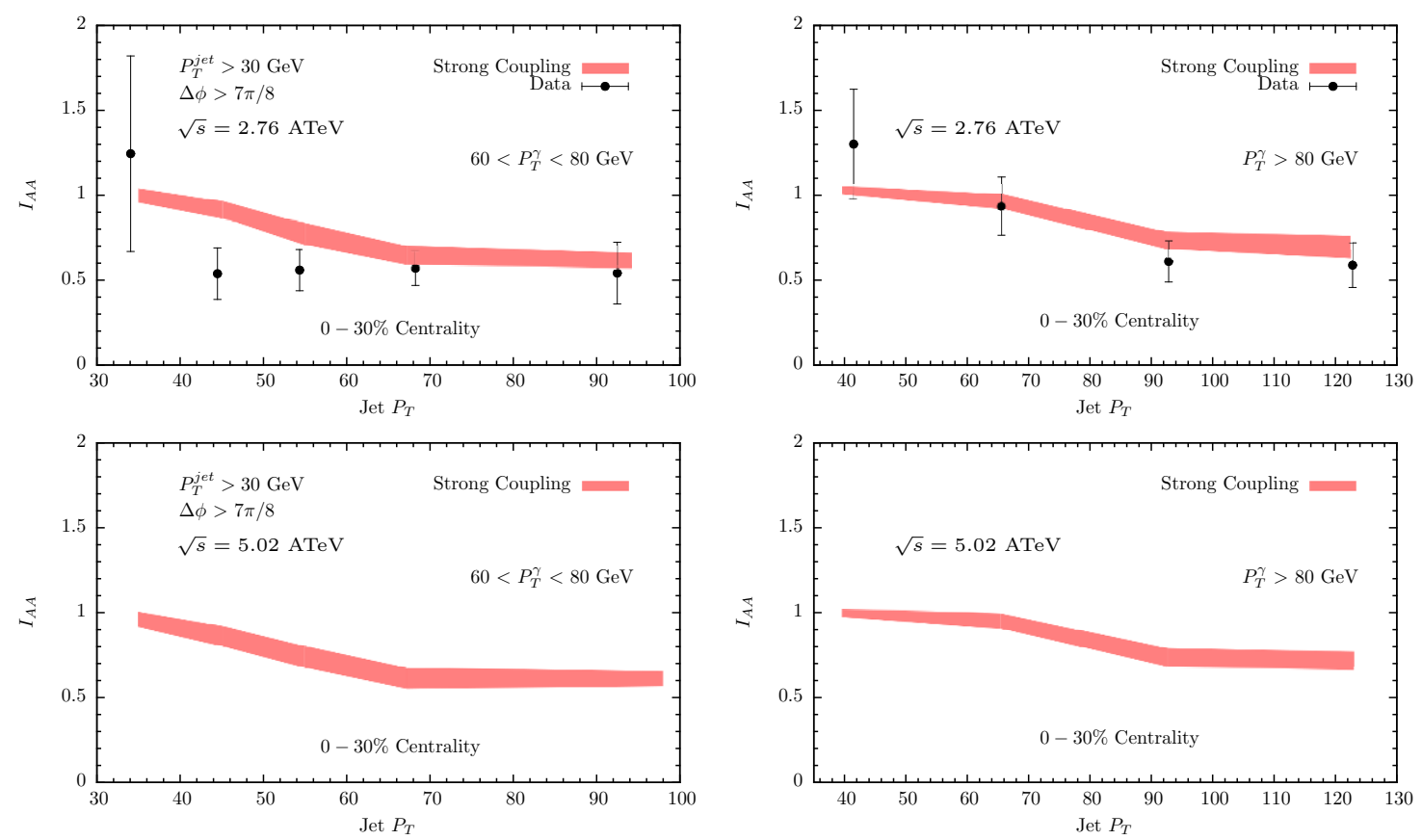

Figure 5. Ratio of the transverse momentum spectra of jets associated with an isolated photon in $\mathrm{Pb}-\mathrm{Pb}$ collisions to that in $\mathrm{p}-\mathrm{p}$ collisions, as a function of the jet transverse momentum, for two different ranges of the photon transverse momentum and for $\sqrt{s}=2.76 \mathrm{ATeV}$ (upper panels) and $\sqrt{s}=5.02 \mathrm{ATeV}$ (lower panels). The hybrid model with its strongly coupled form for the rate of energy loss describes the available CMS data at $\sqrt{s}=2.76 \mathrm{ATeV}$ well.

momenta, $60 \mathrm{GeV}<p_{\mathrm{T}}{ }^{\gamma}<80 \mathrm{GeV}$ and ${p_{\mathrm{T}}}^{\gamma}>80 \mathrm{GeV}$. The observable $I_{A A}$, and in particular its suppression below 1 , can be thought of as the photon-jet analogue of the single-jet suppression $R_{A A}$, but instead of being constructed for inclusive jets as $R_{A A}$ is, $I_{A A}$ is constructed using only the associated jets back-to-back with an isolated photon. This implies that the distribution of the jet energies as well as the fragmentation pattern and hence the jet masses, of the p-p jets and of the $\mathrm{Pb}-\mathrm{Pb}$ jets that enter into the calculations of $I_{A A}$ and $R_{A A}$ are different. Furthermore, the distribution of the point in the transverse plane at which the hard scattering event that produces a jet selected in an $I_{A A}$ analysis is quite different from that for the jets selected in an $R_{A A}$ analysis. It is therefore striking that even though we fitted the single parameter in our hybrid model to a single measured value of $R_{A A}$, when we compare the results for the photon-jet $I_{A A}$ obtained from the model with data at $\sqrt{s}=2.76 \mathrm{ATeV}$, displayed in the upper panels of In figure 5 , we see such good agreement, for both ranges of the photon energy and over the whole range of jet $p_{\mathrm{T}}$. In the lower panels of figure 5, we show the predictions from our hybrid model with its strongly coupled rate of energy loss for heavy ion collisions with $\sqrt{s}=5.02 \mathrm{ATeV}$. As for the previous photon-jet observables, the spectrum of the associated jets hardly changes between these two collision energies.

The agreement between the predictions of our hybrid strong/weak coupling model, with its PYTHIA branching and its strongly coupled form for the rate of energy loss, for all of these photon-jet observables and the data available today is very encouraging. Having 
fixed the single parameter in our model using a single measurement of $R_{A A}$ for inclusive jets, without introducing any new parameters we have obtained a good description of the experimental data for a total of 5 different observables, one involving inclusive jets, one involving dijets, and three involving photon-jets, all with their centrality and energy dependence. These observables sample different in-medium path length distributions of the quenched jets, different shapes of the original jet spectrum, different fragmentation patterns and jet mass distributions, and different quark vs. gluon compositions of the observed jets. Despite all these differences, our model is able to describe the systematics observed in all the data correctly. To avoid over-reaching in drawing conclusions, however, it is important to explore the predictions of the different control models described in section 2.1 for these observables. The results of this analysis can be found in appendix D. There are distinctions between the predictions of the control models and our hybrid model, but these distinctions are small compared to the statistical uncertainties in present data. It is therefore not possible at present to use the agreement between our hybrid model and photon-jet data to argue that the data favors a strongly coupled form for the rate of energy loss. We therefore await the higher statistics data expected later this year in collisions with $\sqrt{s}=5.02 \mathrm{ATeV}$, and have provided the predictions of our hybrid model for all three photon-jet observables for collisions at this higher energy. We have also provided such predictions for Z-jet observables below, in section 3.3. In addition, it is important to investigate the predictions of our hybrid model for intra-jet observables like fragmentation functions, as we shall do in section 4 . These are all paths toward using jet data in the service of understanding the dynamics of the interaction between energetic partons and strongly coupled plasma. In particular, once the statistical precision of the experimental data increases we look forward to identifying deviations between the predictions of our hybrid model and various experimental data as this will allow us to begin to see, quantify, and understand the effects of the physical effects that we are leaving out in our present simple, one-parameter, hybrid model.

\subsection{Z-jet observables: predictions for $\sqrt{s}=5.02 \mathrm{ATeV}$}

In this section, we turn to the $\mathrm{Z}^{0}$-jet observables that are complementary to the photon-jet observables of the previous section. The large Z-mass ensures that Z-bosons seen in a heavy ion collision were almost without exception produced promptly, in hard processes dominated by short-distance physics: it is extremely unlikely for a Z-boson to be produced during the fragmentation of a parton in a jet, unless the jet energy is much higher than is relevant to us. The Z-boson production mechanism is therefore under good theoretical control. ${ }^{9}$ Furthermore, because of their large width and short lifetime, Z-bosons decay during the very early stage of a heavy ion collision, even prior to plasma formation. The Z-bosons that are identified in heavy ion collisions are those that decayed leptonically, in particular via $Z^{0} \rightarrow \mu^{+} \mu^{-}$. This means that their decay products do not interact strongly with the pre-equilibrium partonic matter or with the strongly coupled plasma, once it

\footnotetext{
${ }^{9}$ Note, of course, that modifications of the nuclear parton distribution function in the relevant intermediate $x$-region can alter the production rate of Z-bosons, by modifying the composition of the initial flux of partons.
} 

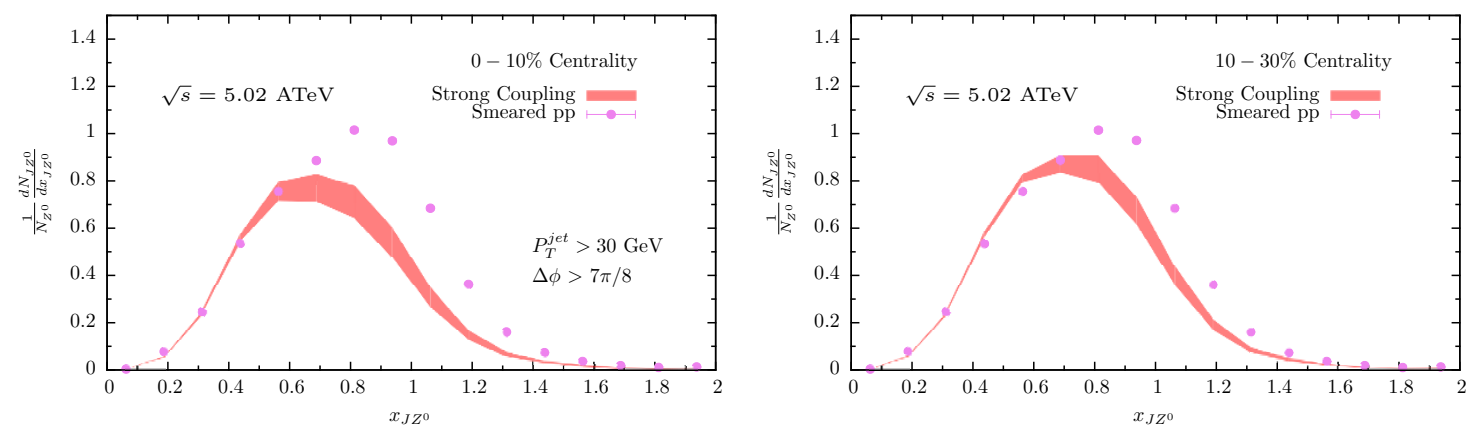

Figure 6. Distribution of the transverse momentum imbalance of Z-jet pairs, $x_{J Z} \equiv p_{\mathrm{T}}{ }^{\text {jet }} / p_{\mathrm{T}}{ }^{\mathrm{Z}}$, for $\mathrm{Pb}-\mathrm{Pb}$ collisions with $\sqrt{s}=5.02 \mathrm{ATeV}$. The left and right panels show the $0-10 \%$ and $10-30 \%$ most central events, respectively. Here and below, the colored bands show the results from our hybrid model, with its strongly coupled form for the rate of energy loss and the violet dots show the smeared p-p calculations for comparison.

forms. Z-bosons are in this respect similar to prompt photons, making Z-jet events similar in their utility to photon-jet events. The Z-jet events come with the added advantage almost all Z's are prompt Z's. As consequence, the experimental identification of Z-bosons via their $\mu^{+} \mu^{-}$decays do not require isolation cuts, which leads to an arguably cleaner determination of the associated jet energy. The only disadvantage of Z-jet events is that Z's are less numerous than photons.

We construct the same class of observables for Z-jet events that we constructed for photon-jet events in the previous section. Because of the low statistics of Z-boson production in $\sqrt{s}=2.76 \mathrm{ATeV}$ collisions, no constraining measurements exist at present for the Z-jet observables that we shall construct. ${ }^{10}$ We therefore present predictions for these observables in the $\sqrt{s}=5.02 \mathrm{ATeV}$ heavy ion collisions coming soon in LHC heavy ion Run 2. We have chosen the same kinematical cuts for the Z-jet observables that we (and the CMS collaboration) have used in photon-jet observables, to facilitate comparison between our results for the two cases. Of course, once the experimentalists decide on the cuts that they will use for their Z-jet analyses of the data-to-come, we can re-run our analyses with their cuts.

In figure 6, we show the distribution of the Z-jet imbalance observable $x_{J Z} \equiv p_{\mathrm{T}}{ }^{\text {jet }} / p_{\mathrm{T}}{ }^{\mathrm{Z}}$, in heavy ion collisions with two different ranges of centrality with $\sqrt{s}=5.02 \mathrm{ATeV}$. In both cases the colored band shows the predictions of our hybrid model, with its strongly coupled form (2.1) for the rate of energy loss, for $\mathrm{Pb}-\mathrm{Pb}$ collisions and the violet dots show the distribution of $x_{J Z}$ for p-p collisions as predicted by PүтніA. As we did in our analysis of photon-jet observables, we have smeared the momenta of the associated jets in both our $\mathrm{Pb}-\mathrm{Pb}$ and $\mathrm{p}-\mathrm{p}$ calculations. For the present, before better guidance becomes available once experimentalists have begun the analysis of LHC Run 2 data, we have used the same smearing functions here as we (and the CMS collaboration) used for the photon-jet observables that we discussed in the previous section. We obtained the $x_{J Z}$ distributions in figure 6 from a sample of events in which we required a Z-boson with $p_{\mathrm{T}}{ }^{Z}>60 \mathrm{GeV}$

\footnotetext{
${ }^{10}$ For preliminary low-statistics measurements of Z-jet correlations at $\sqrt{s}=2.76 \mathrm{ATeV}$, see ref. [116].
} 


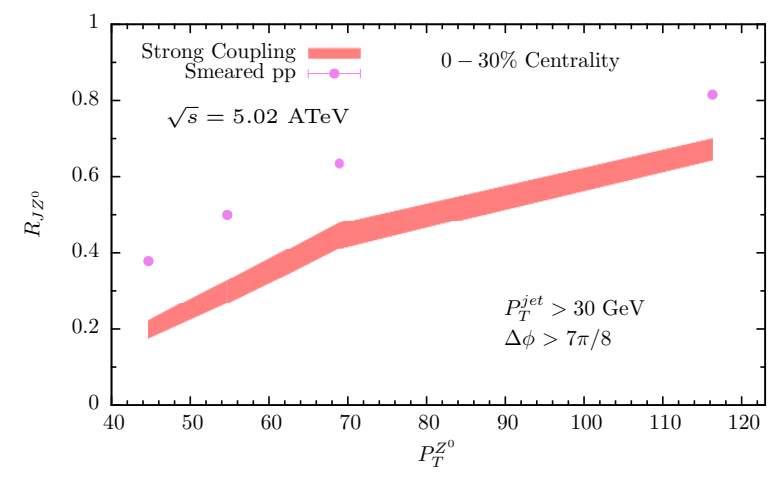

Figure 7. Fraction of events with a Z-boson in which we find a Z-jet pair, which is to say in which we find an associated jet with $p_{\mathrm{T}}^{\text {jet }}>30 \mathrm{GeV}$ at an azimuthal angle more than $7 \pi / 8$ away from that of the Z-boson, in collisions with $\sqrt{s}=5.02 \mathrm{ATeV}$.

and an associated jet reconstructed using the anti- $k_{T}$ algorithm with $R=0.3$ that has $p_{\mathrm{T}}{ }^{\text {jet }}>30 \mathrm{GeV}$ and is separated in azimuthal angle from the Z-boson by $\Delta \phi>7 \pi / 8$. We required that both the $\mathrm{Z}$ and the associated jet have $|\eta|<1.6$. As in the case of the $x_{J \gamma}$ distribution in photon-jet events, the $x_{J Z}$ distribution is broad in p-p collisions, indicating the importance of events with a $\mathrm{Z}$ and two jets, in particular those arising from initial state radiation. This means that at present Z-bosons are not substantially better as taggers of the associated jet energy than photons are, which motivates the future development of methods to suppress events with more than one jet in the final state. Because the transverse momentum of the $Z$-boson and its $\mu^{+} \mu^{-}$decay products do not change in the medium, the difference between the $\mathrm{Pb}-\mathrm{Pb}$ distribution and the p-p distribution in our calculation is entirely due to the energy lost by the partons in the associated jet in the $\mathrm{Pb}-\mathrm{Pb}$ collisions due to their passage through the strongly coupled plasma. As for photon-jet events, we see a reduction in the integral of $x_{J Z}$, analyzed further below, and a displacement of the distribution toward smaller $x_{J Z}$. The magnitude of this displacement is comparable to the corresponding shift in the $x_{J \gamma}$ distribution in photon-jet events, see figure 3 . As in that case, there are clear but small distinctions between the results we obtain with our hybrid model, shown in figure 6 , and those we obtain when we use our control models for the rate of energy loss (2.2) instead. We present these in appendix D.

In figure 7 , we compute the fraction of Z-bosons in our sample that come with an associated jet, as reconstructed with the anti- $k_{T}$ algorithm with $R=0.3$, that has $p_{\mathrm{T}}{ }^{\text {jet }}>$ $30 \mathrm{GeV}$ and $\Delta \phi>7 \pi / 8$. We plot this quantity, which we denote by $R_{J Z}$, as a function of the transverse momentum of the Z-boson. We show the results obtained from our smeared proton-proton PYTHIA simulations as the violet dots and the predictions for $\mathrm{Pb}$ $\mathrm{Pb}$ collisions from our hybrid strong/weak coupling model as the colored band. As in the photon-jet case, as the partons in the associated jet shower lose energy the total energy of the associated jet can drop below our $p_{\mathrm{T}}{ }^{\text {jet }}=30 \mathrm{GeV}$ cut, meaning that energy loss leads to a reduction of the Z-jet yield as a fraction of the number of Z-bosons. This makes the integral under the colored bands in figure 6 less than that under the violet dots there, and it pushes the colored band in figure 7 below the violet dots. The qualitative behavior and 

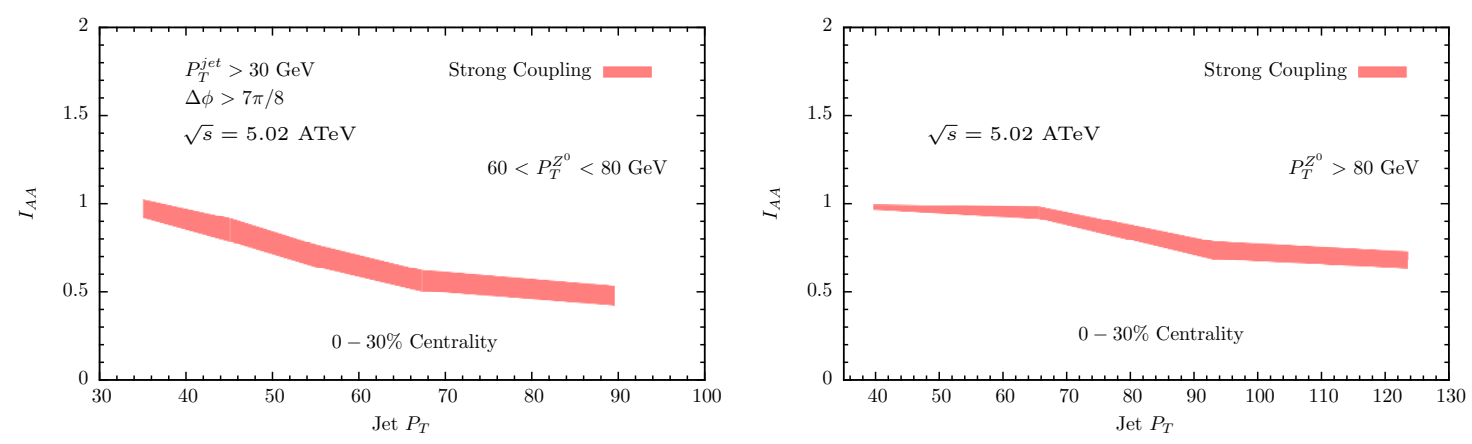

Figure 8. Ratio of the transverse momentum spectra of jets associated with a Z-boson in Pb$\mathrm{Pb}$ collisions with $\sqrt{s}=5.02 \mathrm{ATeV}$ to that in p-p collisions as a function of the jet transverse momentum, for two different ranges of the Z-boson transverse momentum.

magnitude of this reduction is comparable to the reduction in the analogous photon-jet observable, see figure 4.

Finally, in figure 8 we analyze the spectrum of jets produced in association with a Z-boson. We show the ratio of the spectrum of associated jets in $\mathrm{Pb}-\mathrm{Pb}$ collisions to that in p-p collisions, $I_{A A}$, for two different ranges of Z-boson transverse momenta, $60 \mathrm{GeV}<$ $p_{\mathrm{T}}{ }^{\mathrm{Z}}<80 \mathrm{GeV}$ and ${p_{\mathrm{T}}}^{\mathrm{Z}}>80 \mathrm{GeV}$. The effect of energy loss on this observable is again comparable to its effects on the analogous photon-jet observable, see figure 5 .

In appendix $\mathrm{D}$, we repeat the analysis of $R_{J Z}$ and $I_{A A}$ for jets produced in association with a Z-boson in our control models, where we use the expressions (2.2) for the rate of energy loss. We find that these observables exhibit little sensitivity to the form of $d E / d x$, meaning little sensitivity to the microscopic dynamics via which the partons in the jet shower interact with the strongly coupled plasma.

In sections 3.2 and 3.3, we have provided predictions for three photon-jet observables and three Z-jet observables in heavy ion collisions with $\sqrt{s}=5.02 \mathrm{ATeV}$ obtained from our hybrid strong/weak coupling model, with fragmentation taken from PYTHIA and a rate of energy loss (2.1) as at strong coupling. We await the data that will come from LHC heavy ion Run 2 with considerable anticipation.

\section{Fragmentation functions}

We now turn to the analysis of a more differential class of jet observables, namely fragmentation functions. We saw in ref. [23] and have confirmed in figure 17 of appendix B that the predictions of our hybrid model and of our two control models for the ratio of the partonic fragmentation function of inclusive jets in $\mathrm{PbPb}$ collisions to that in $\mathrm{p}$-p collisions are distinct for the three models. This motivates the hope that the higher statistics measurements expected from the coming LHC run may serve to distinguish between models. And, it motivates us to compute the predictions of all three models for the partonic fragmentation functions of jets produced in association with an isolated photon or a Z-boson. We shall present the results of these calculations in section 4.1. We were initially surprised to see that the partonic fragmentation function ratio for jets produced in association with bosons 
turn out to be more similar for the three models we are considering than was the case for inclusive jets. Understanding this effect, which we shall do by the end of section 4.1, leads us, in section 4.2, to introduce a new observable constructed from the fragmentation functions of jets in dijet pairs in $\mathrm{Pb}-\mathrm{Pb}$ collisions, without the need for any $\mathrm{p}-\mathrm{p}$ reference. This turns out to be the observable that is most effective at differentiating among our three models of any observable that we have considered to date.

Unlike the more inclusive observables described in the previous section, the hadronic fragmentation functions that experimentalists measure are quite sensitive to hadronization effects. However, the dynamics of hadronization, even in vacuum, are not under full theoretical control. In fact, the predictions of different Monte Carlo event generators for fragmentation functions can differ among themselves, and in comparison with p-p data, by as much as $20 \%$ as shown in refs. [117, 118]. Since the modification of the fragmentation functions in $\mathrm{Pb}-\mathrm{Pb}$ collisions with respect to those in $\mathrm{p}-\mathrm{p}$ collisions is itself on the order of several tens of percent at most (see the measurements reported in ref. $[9,15,119]$ ) and since there are differences in hadronization dynamics (in particular, differences in the patterns of color recombination) for jets in $\mathrm{Pb}-\mathrm{Pb}$ and p-p collisions [120-122], it will be challenging to compare the ratios of partonic fragmentation functions in $\mathrm{Pb}-\mathrm{Pb}$ collisions to those in p-p collisions - ratios which we calculate in our models in section 4.1 - to data. The in-medium effects of interest are comparable in magnitude to the known uncertainties coming from our lack of understanding of hadronization dynamics. This is strong further motivation for the importance of the observable that we introduce in section 4.2: the ratio of the fragmentation function of inclusive jets in $\mathrm{Pb}-\mathrm{Pb}$ collisions to that of the associated jets in dijet pairs. Since this is the ratio of the fragmentation functions of two different classes of jets in $\mathrm{Pb}-\mathrm{Pb}$ collisions, with no need for a p-p reference, many hadronization uncertainties will cancel.

We shall restrict our calculations to partonic fragmentations throughout this section, meaning that our calculations are not sensitive to hadronization and so are not affected by its challenges. The observable that we introduce in section 4.2 is the one in this section for which this will to the greatest degree possible also be true in experimental data. For this observable, as for those we calculate in section 4.1, however, the fragmentation functions describing fragments with the lowest $p_{\mathrm{T}}$ 's cannot be described reliably by our hybrid model or by the two control models because none of these models include the contribution to the low- $p_{\mathrm{T}}$ component of a jet arising from the wake in the recoiling plasma that the jet plowing through it produces [47, 123-127].

\subsection{Fragmentation functions of the associated jets in photon-jet and Z-jet pairs}

Fragmentation functions are defined as the distribution of hadrons within a jet with a given fraction $z$ of the total longitudinal momentum $p_{\|}$of the jet. Longitudinal, here, means in the direction of the jet axis. In p-p collisions, where most of the activity in events with hard jets comes from hadrons produced via the fragmentation and subsequent hadronization of the virtual partons produced in an initial elementary partonic collision, fragmentation functions provide us with information about how the showering process via which the large virtuality of the initial partons relaxes takes place. Furthermore, since 
final state effects are negligible, $p_{\|}$obtained from the energy of a jet reconstructed with a sufficiently large reconstruction radius provides a good proxy of the initial energy of the hard parton that fragmented to form the jet. This means that the $z$-fraction of the final fragments are directly related to one of the QCD evolution variables. This is not the case in $\mathrm{Pb}-\mathrm{Pb}$ collisions.

We have already mentioned one of the complications in $\mathrm{Pb}-\mathrm{Pb}$ collisions: at low $p_{\mathrm{T}}$, some of the particles in a reconstructed jet did not originate from the initial hard parton that was produced in the initial elementary partonic collision. Some soft particles reconstructed in the jet come, instead, from the hadronization of moving quark-gluon plasma, set in motion by the momentum that the jet passing through it transfers to the medium through which it is passing. Operationally, this enters the analysis because in analyzing jets in $\mathrm{Pb}-$ $\mathrm{Pb}$ collisions it is necessary and standard to do a background subtraction to remove the hadrons formed from the quark-gluon plasma, and this background subtraction procedure is based upon the assumption that the momenta of these hadrons is uncorrelated with the direction of the jet. To the extent that this is the case, the background subtraction removes, on a statistical basis, particles in the jet cone that are not part of the jet itself. However, since the interaction of the jet with the plasma transfers momentum to the plasma, this back reaction (or recoil) effect means that the background subtraction procedure cannot remove all the particles from the medium: there is no way to disentangle all of them from the products of the jet shower; some of them must end up incorporated into jet observables. This means that, in $\mathrm{Pb}-\mathrm{Pb}$ collisions, fragmentation functions at low $p_{\mathrm{T}}$ are sensitive to physical processes other than jet fragmentation. Addressing these additional physical processes requires a dynamical treatment of the response of the medium to the passage of the jet, which is beyond our current model implementation. For this reason, the results of our calculations become less reliable at small $z$. In this section, we will look at jets with $p_{\mathrm{T}}>30 \mathrm{GeV}$, meaning that $p_{\|}>30 \mathrm{GeV}$. So, $\ln (1 / z)=2.7$ or $\ln (1 / z)=3.5$ corresponds to fragments with $p_{\mathrm{T}}>2 \mathrm{GeV}$ or $p_{\mathrm{T}}>1 \mathrm{GeV}$. We will plot our results out to larger values of $\ln (1 / z)$, smaller values of $z$, but the effects of medium recoil that we are not including become more and more important for $\ln (1 / z) \gtrsim 3$. In section 4.2 where we consider inclusive jets and dijets, rather than jets produced in association with a boson, we will look at jets with $p_{\mathrm{T}}>80 \mathrm{GeV}$, meaning that our results there will be reliable out to somewhat larger values of $\ln (1 / z)$.

There is a second complication in interpreting jet fragmentation functions in $\mathrm{Pb}-\mathrm{Pb}$ collisions, and this is that the $p_{\|}$of a jet, as reconstructed from the final state, is less than the energy of the initial hard parton that fragmented into the jet because the partons in the jet have lost energy as they propagate through the strongly coupled medium produced in the $\mathrm{Pb}-\mathrm{Pb}$ collision. At least some of the "lost" energy (according to the data $[6,128]$, a significant amount of it) ends up as soft particles moving at large, random, angles relative to the jet axis, and is not included when the jet is reconstructed. Therefore, even when they are reconstructed with a large reconstruction radius, the total energy of the quenched in-medium jets is smaller than the total energy of the hard partons originating from an elementary partonic collision. This means that when a jet is reconstructed in a $\mathrm{Pb}-\mathrm{Pb}$ collision, it is impossible to make an experimental determination of the energy of the initial hard parton. Consequently, if one constructs a fragmentation function using the standard 
definition of $z$, namely the ratio between the momentum of an individual hadron to the $p_{\|}$ of the whole jet as reconstructed, this $z$ is not directly related to the evolution variable in a DGLAP shower and, more generally, is simply hard to interpret. We will nevertheless report the predictions of our hybrid model and the two control models for fragmentation functions computed in this standard way, for comparison to future data.

Because we are looking at jets produced in association with a photon or Z-boson, however, there is an obvious alternative. We can define $z_{\gamma}$ or $z_{Z}$ (we shall denote these variables generically as $z_{B}$ ) as the ratio of the momentum of an individual hadron in the jet to the momentum of the $\gamma$ or Z-boson, using the momentum of the electroweak boson in the event (which cannot have lost any energy since it does not interact with the quark-gluon plasma) as a proxy for the momentum of the initial hard parton that later fragmented and lost energy, forming the jet that the experimentalists reconstruct. ${ }^{11}$ This is an improvement but it is not a panacea: we have seen in the previous section that even in p-p collisions there is a broad distribution of the momentum imbalance between a boson (photon or $\mathrm{Z}$ ) and the associated jet in a boson-jet event. One significant contributor to this imbalance is the fact that in many events the elementary hard scattering process produces one photon or $\mathrm{Z}$ plus more than one hard parton, not just one. Regardless, this imbalance is entirely due to perturbative vacuum QCD physics, not to any in-medium effects. This means that even when we construct fragmentation functions using $z_{\gamma}$ or $z_{Z}$, we are not reliably dividing by the actual momentum of the initial hard parton that fragmented into the jet we are looking at. Still, by using $z_{\gamma}$ or $z_{Z}$ in $\mathrm{Pb}-\mathrm{Pb}$ collisions we are using a variable that is as good a proxy for what we want as is the case in the standard fragmentation function in p-p collisions.

We first compute the fragmentation functions constructed with respect to the reconstructed energy of the jet produced in association with a photon or Z-boson. (We use the standard variable $z$, but here we denote it $z_{J}$ to emphasize that its denominator is the momentum of the reconstructed jet. It is defined by $z_{J} \equiv p_{\|}^{\text {parton }} / p_{\|}^{\text {jet }}$.) In figure 9 we plot the ratio of the fragmentation functions in $\mathrm{Pb}-\mathrm{Pb}$ to $\mathrm{p}-\mathrm{p}$ as obtained in our framework for jets of $p_{\mathrm{T}}>30 \mathrm{GeV}$ produced in association with isolated photons (upper panels) and Z-bosons (lower panels) with $p_{\mathrm{T}}>60 \mathrm{GeV}$. We have performed these simulations for two different $\mathrm{Pb}-\mathrm{Pb}$ centralities, $0-10 \%$, shown on the left panels and $10-30 \%$, shown on the right. Both photons and Z-bosons are required to have $|\eta|<1.4$ while the jets are constrained to $\left|\eta_{\text {jet }}\right|<1.6$. The energy and direction of the jet axis are reconstructed with the anti- $k_{T}$ algorithm with radius $R=0.3$. Following the experimental analyses of inclusive fragmentation functions in ref. [15], these fragmentation functions are constructed by including all particles surrounding the jet axis within an angular distance in $\eta$ - $\phi$ space of $r=0.3$. Since our simulations do not include the underlying event, these correspond to particles from the hard scattering process that remain correlated with the jet direction. (As noted above, in the analysis of experimental data a background subtraction is done, but soft particles from the plasma that is in motion following the jet will not be subtracted. This means that our calculations should not reproduce the data-to-come at large values of $\ln \left(1 / z_{J}\right)$.)

\footnotetext{
${ }^{11}$ See ref. [47] for a similar definition of the scaling variable.
} 

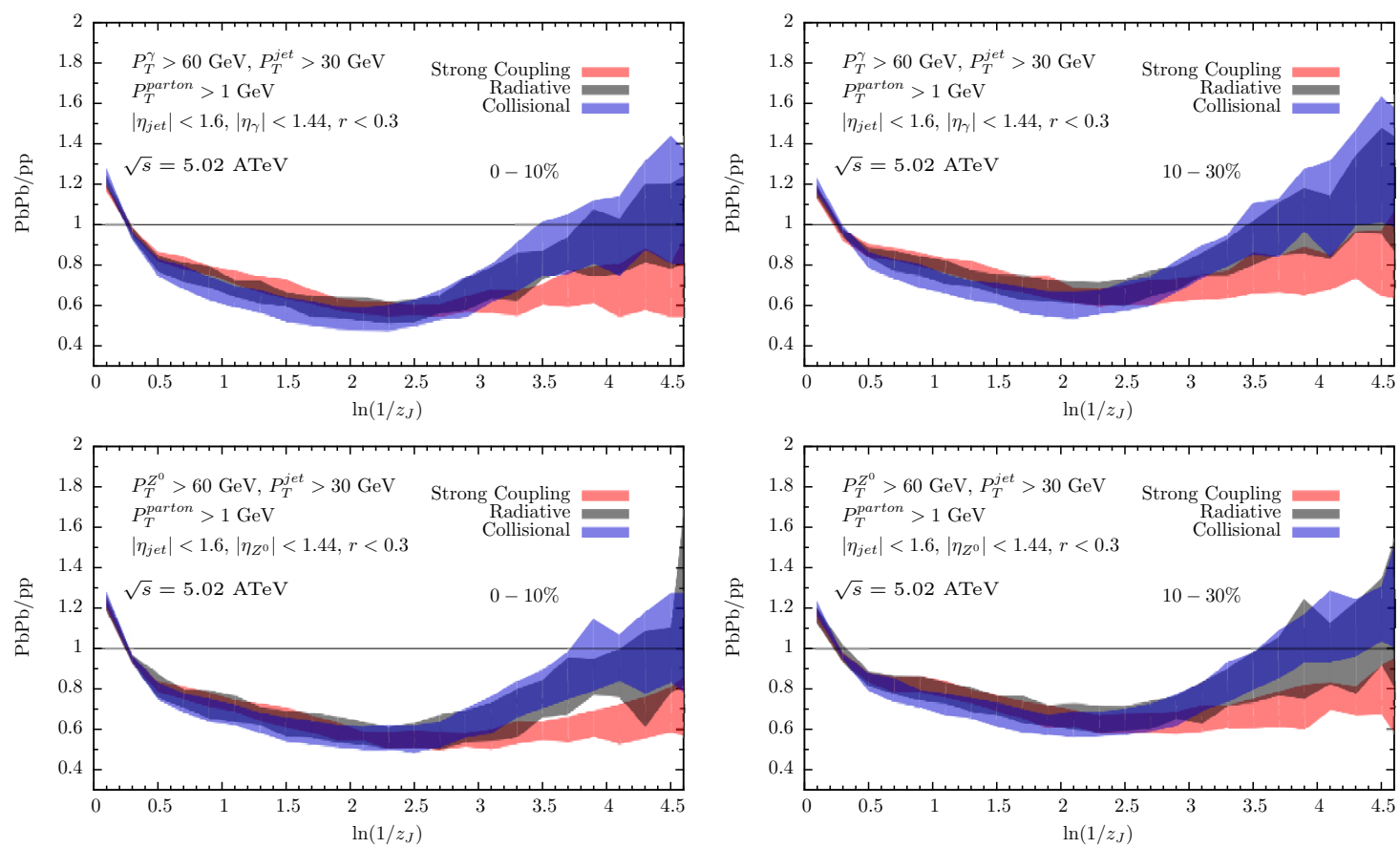

Figure 9. Predictions of our hybrid model, with strongly coupled energy loss for the partons in a Pythia shower, as well as our two control models for the partonic fragmentation function ratios (fragmentation function for jets in $\mathrm{Pb}-\mathrm{Pb}$ collisions over that for jets in $\mathrm{p}$-p collisions) for jets produced in association with an isolated photon (upper panels) or a Z-boson (lower panels) in $\mathrm{Pb}-\mathrm{Pb}$ collisions at $\sqrt{s}=5.02 \mathrm{ATeV}$ at two centralities (left and right panels). The fragmentation functions are constructed with respect to the variable $z_{J}=p_{\|}^{\text {parton }} / p_{\|}^{\text {jet }}$.

The general features of the fragmentation function ratios plotted in figure 9 are very similar to those that we found for inclusive jets in ref. [23] and have confirmed in figure 17 of appendix B. All the models display an enhancement of the hardest part of the fragmentation function in $\mathrm{Pb}-\mathrm{Pb}$ collisions relative to $\mathrm{p}-\mathrm{p}$ collisions. This is a generic behavior of any mechanism that removes soft particles from the jet, either via energy loss as here or via deflecting them into a direction far from that of the jet [129]. Removing soft particles increases the fraction of jets with a few hard fragments, which leads to the increase in the hard part of the fragmentation function. For all models there is also a depletion in the $\mathrm{Pb}-\mathrm{Pb}$ fragmentation function at intermediate $z_{J}$. This is the expected result from quenching, which tends to reduce the energy of the fragments that propagate in plasma. Remarkably, for the energy range of bosons and jets explored in those figures, and with our current uncertainties, the pattern of fragmentation at large and intermediate $z_{J}$ is indistinguishable among the three models we explore, despite their very different path length and energy dependences. We comment further on this below. At smaller values of $z_{J}$, the hybrid model with its strongly coupled energy loss suppresses soft fragments more than the control models. However, this separation between models occurs in a regime where the fragments have momenta smaller than $2 \mathrm{GeV}$, meaning that our calculations of fragmentation functions are not reliable there. Adding in the contributions from a medium 

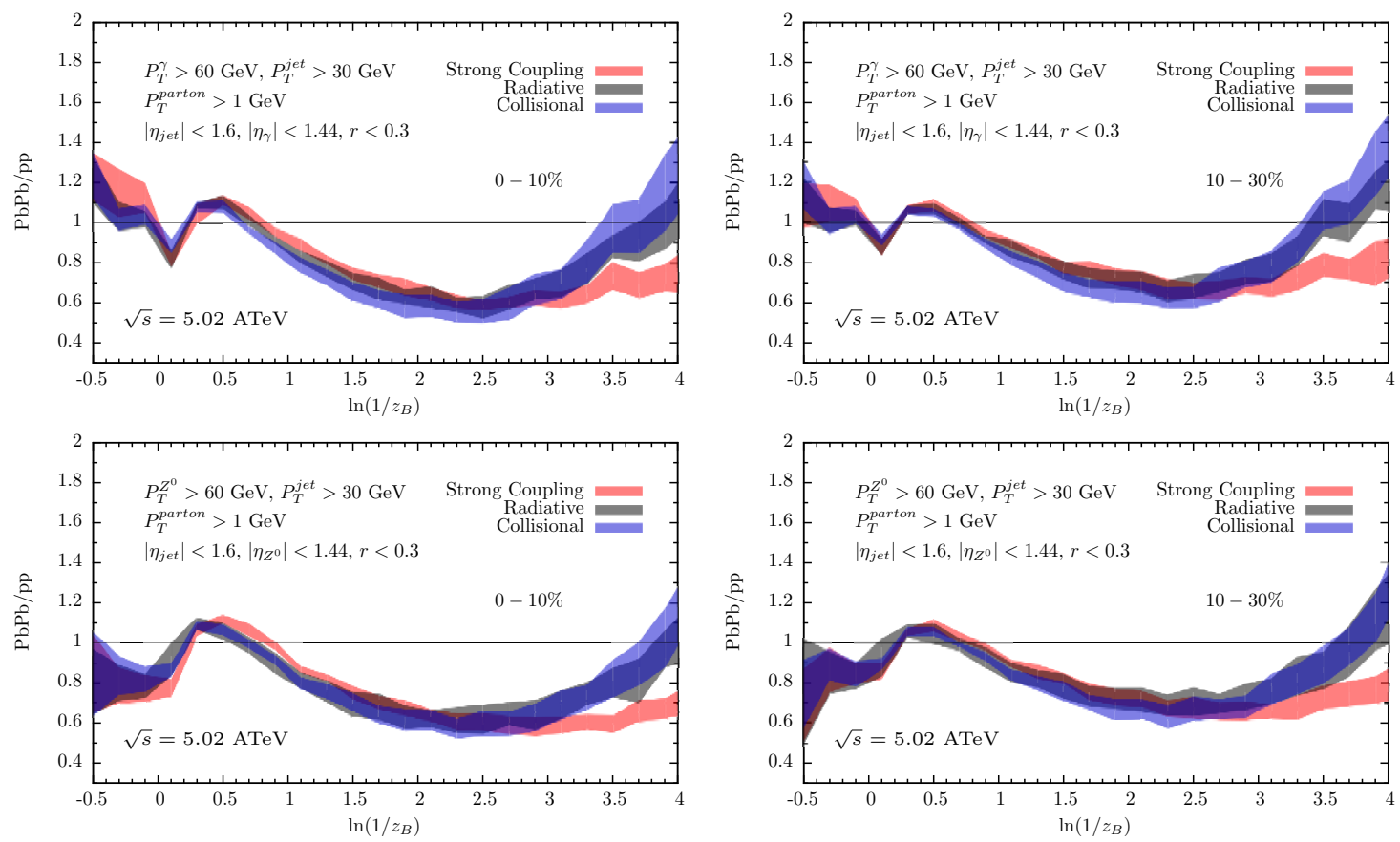

Figure 10. Predictions of our hybrid model and our two control models for the partonic fragmentation function ratios $(\mathrm{Pb}-\mathrm{Pb}$ over $\mathrm{p}-\mathrm{p})$ for jets produced in association with a boson as a function of $z_{B}=-\mathbf{p}_{T}^{\text {parton }} \mathbf{p}_{T}^{B} /\left(p_{\mathrm{T}}^{B}\right)^{2}$ for two different centralities for photon-jet (upper) and Z-jet (lower) events in $\mathrm{Pb}-\mathrm{Pb}$ collisions at $\sqrt{s}=5.02 \mathrm{ATeV}$.

that has picked up momentum from the jet passing through it, meaning that it is not completely removed by the background subtraction, would push the $\mathrm{Pb}-\mathrm{Pb}$ fragmentation functions up in this soft region by an amount that our model does not permit us to estimate at present.

In figure 10 we reanalyze the fragmentation function ratios, this time using the boson momentum $z_{B}$ to define the scaling variable according to $z_{B} \equiv-\mathbf{p}_{T}^{\text {parton }} \mathbf{p}_{T}^{B} /\left(p_{\mathrm{T}}{ }^{B}\right)^{2}$, with $\mathbf{p}_{T}^{B}$ the transverse momentum of the isolated photon or Z-boson. ${ }^{12}$ As already mentioned, our main motivation for redefining the scaling variable is to have a better proxy for the jet energy prior to quenching. If all bosons were prompt, their momentum would be insensitive to in-medium effects and energy loss would only affect the numerator of $z_{B}$. For those prompt bosons, the mismatch between the boson and the initiator parton of the jet originates entirely in vacuum processes. This is the case for $Z$-jet correlations, where the possibility of producing a $Z$ in a jet shower is highly suppressed. In contrast, in the isolated photon sample we use, there is a small fraction of fragmentation photons even after we make our isolation cut, and for these photons energy loss effects are present via the quenching of their parent parton. Therefore, for photons, there is also a small

\footnotetext{
${ }^{12}$ Because of the fluctuations of the rapidity of the centre of mass of the elementary partonic collision that leads to boson-jet events, the rapidity of the boson and the jets do not need to be correlated, unlike the transverse momentum. For this reason, to construct $z_{B}$ we have chosen to use only transverse momenta and chosen not to project the momenta of fragments along the boson direction.
} 
dependence of the denominator of $z_{B}$ on quenching; the effect of this small dependence on the fragmentation function ratio depends on the $z_{B}$ range.

Note that as a consequence of the broad distribution of the boson-jet imbalance even in p-p collisions, $z_{B}$ can be larger than one: there are jets with $p_{\mathrm{T}}$ larger than the momentum of the boson they are associated with. Those events populate the region of negative $\log 1 / z_{B}$ in figure 10. In this region, all models lead to an enhancement of the in-medium fragmentation function of jets associated to photons. At first this seems puzzling, but there are in fact two reasons for this effect. The first is the fact that jet quenching does reduce the energy of fragmentation photons, as their parent partons lose energy. The second reason comes from imposing an isolation cut on photons in the events in our calculations, which do not include the particles corresponding to the medium. Since quenching affects all partons in our events, prompt photons in events with more than one jet are more isolated in our $\mathrm{Pb}-\mathrm{Pb}$ simulations than in vacuum. This leads to an enhancement of the fragmentation function ratio for jets produced in association with isolated photons in the region of $x_{J}>1$. However, it is not clear to us whether this effect will persist in a full simulation of photon-jet events in which particles from the $\mathrm{Pb}-\mathrm{Pb}$ background are incorporated in the sample. We leave the study of the fate of this enhancement for future work. For the $Z$-jet correlation neither of these two effects are present, and the negative $\log z_{B}$ region is slightly suppressed, as expected.

The ratio of fragmentation functions also exhibits a non-trivial structure in the vicinity of $\log 1 / z_{B} \sim 0.5\left(z_{B} \sim 0.6\right)$. This structure is correlated with the position of the maximum of the in-medium boson-jet imbalance in figures 3 and $6, x_{J \gamma} \sim 0.6$ and $x_{J Z} \sim 0.6$. Since the maximum of the in-medium imbalance distribution is shifted towards smaller $x$ values, the non-monotonic behavior of the fragmentation function ratio in this region reflects the behavior of the imbalance distribution. Indeed, the relative abundance of associated jets with $x_{J \gamma} \sim 0.6$ or $x_{J Z} \sim 0.6$ is enhanced in $\mathrm{Pb}-\mathrm{Pb}$ collisions with respect to $p-p$. The fact that all three models exhibit the same behavior is a consequence of the coincidence of the imbalance distribution in the three models.

As for fragmentation functions constructed with the variable $z_{J}$, here too the strongly coupled model only separates from the control models for soft particles where the physics of how the medium responds to the passage of the jet, physics that none of our models includes, becomes important.

In summary, the analysis of the fragmentation functions of jets produced in association with photons and Z-bosons indicates that mechanisms of energy loss that do not increase the number of hard fragments in jets, like the ones we have explored, lead to robust modifications to the fragmentation pattern of these jets. This also means that this type of observable is not very sensitive to the microscopic mechanism of parton energy loss.

We close this section by recalling our initial motivation - the separation between the predictions of our models for the fragmentation functions of inclusive jets seen in ref. [23] and in figure 17 - and asking why that separation between model predictions is less in the fragmentation functions for jets produced in association with photons and Z-bosons seen in figures 9 and 10. The answer comes in understanding the selection effects in a sample of "inclusive jets". Because the jet production spectrum is a steeply falling function of $p_{\mathrm{T}}$, 
and because usually two or more jets are produced in an event, most of the jets in a sample of inclusive jets are the most energetic jet in an event. In selecting a sample of inclusive jets, one is preferentially selecting jets that are the jet in their event that has lost the least energy. In contrast, when one selects jets by first identifying an isolated photon or Z-boson and then reconstructing an associated jet there is no such selection effect. This means that, on average, the inclusive jets whose fragmentation functions are shown in figure 17 have travelled through the medium over a shorter path-length and, again on average, they are jets that fragmented less. Fewer fragments, i.e. a jet with a lower jet mass and a smaller opening angle and a harder fragmentation function, means that within the jet there are fewer partons losing energy in the medium, and therefore means less energy loss. Both these effects are likely small compared to the event-by-event variation. But, on average, inclusive jets contain somewhat fewer fragments ${ }^{13}$ and traverse somewhat less plasma. What are the consequences in our models of the fact that in going from figure 17 to figures 9 and 10 there is an increase in path length? The path-length dependence of $d E / d x$ in (2.1) is stronger than in the control models (2.2) meaning that in the mid-range of $z$ where we see the effects of quenching (say $1<\log 1 / z<3$ ) we expect the increase in path length to push the predictions of our hybrid strongly coupled model down relative to the control models. What are the consequences of the fact that in going from figure 17 to figures 9 and 10 the jets become wider and there is some reduction in the energy of the partons within them? In (2.1), a reduction in the energy of the partons means a reduction in the stopping length $x_{\text {stop }}$ and an increase in $d E / d x$. So, for this reason also we expect to see the predictions of our hybrid strongly coupled model pushed down relative to those of the control models. And, this is indeed what we see. Unfortunately, the predictions of the hybrid model get pushed down just to the extent that in figures 9 and 10 they are essentially on top of the predictions of the control models.

In the next section, we show that it is possible to select a sample of jets in which the predictions of the hybrid strongly coupled model for the fragmentation functions in the mid-range $1<\log 1 / z<3$ are pushed down even farther.

\subsection{Fragmentation functions of the associated jets in dijet pairs}

Motivated by the results and discussion above, we now turn our attention to the fragmentation functions of the associated (less energetic) jets in dijet events. Whereas in the inclusive jet sample of figure 17 we have selected jets that are likely to be those among the jets in their event that have been quenched the least, by selecting associated jets in dijet events we will likely be selecting those that have been quenched the most. That means we will be selecting those that have, on average, traversed a longer path-length of medium and those that were produced, on average, with a larger jet mass and jet opening angle and that, on average, contained more, and therefore lower energy, fragments before quenching.

\footnotetext{
${ }^{13}$ There are actually two reasons why they contain somewhat fewer fragments. First, as we shall see only in the next section, even in p-p collisions the higher energy jet in a dijet pair — and this is what most jets in an inclusive jet sample are - tends to have a harder fragmentation function. And, second, jets with fewer, harder, fragments lose less energy [130] in the plasma produced in a $\mathrm{Pb}-\mathrm{Pb}$ collision and so are even more likely to end up being the higher energy jet in a dijet pair.
} 

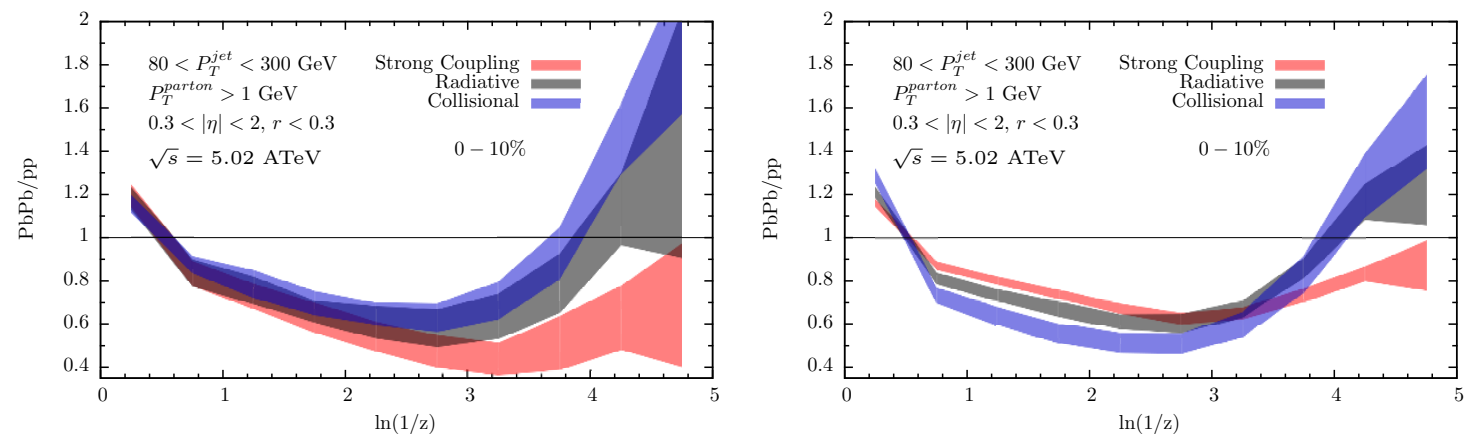

Figure 11. Left: model predictions for the $\mathrm{Pb}-\mathrm{Pb}$ to $\mathrm{p}-\mathrm{p}$ ratio of fragmentation functions of associated jets (lower energy jets) in dijet events at $\sqrt{s}=5.02 \mathrm{ATeV}$ with a leading jet of $p_{\mathrm{T}}>$ $120 \mathrm{GeV}$. Right: same for inclusive jets with the same range of $p_{\mathrm{T}}$ and $\eta$ as the associated jets in the left panel. The right panel is similar to figure 17, but is for a different $p_{\mathrm{T}}$ range and is for the higher LHC heavy ion Run 2 collision energy.

In the left panel of figure 11, we show the ratio of fragmentation functions for jets of $80<p_{\mathrm{T}}<300 \mathrm{GeV}$ with $0.3<|\eta|<2$ produced in association with a jet of $p_{\mathrm{T}}>120 \mathrm{GeV}$ in $\mathrm{Pb}-\mathrm{Pb}$ collisions to that in p-p collisions. By the associated jet in a dijet pair we will always mean the jet with the lower energy. For comparison, in the right panel of figure 11 we have analyzed inclusive jet fragmentation in a lower energy range, such that the momentum of the inclusive jets is in the same range as the momentum of the associated jets in the left panel of figure 11. Although by careful comparison to figure 17 we see that the modification pattern seems to be slightly dependent on the jet energy, the basic features in the right panel of figure 11 and in particular the ordering of the predictions of the three models, is the same as in figure 17. The inclusive jets are selected in a way that makes them likely to be the less quenched jets in their event with, on average, a shorter path length and fewer fragments. The jets in section 4.1, selected via having been produced in association with photons or Z-bosons, have no such selection effects. And, the associated jets in the left panel of figure 11 are selected in a way that makes them likely to be the more quenched jets in their event with, on average, a longer path length and more fragments. Sure enough, we see that the predictions of our hybrid strongly coupled model — with a $d E / d x$ that depends strongly on path-length and that increases at lower energies as $x_{\text {stop }}$ decreases are pushed lower than those of the control models in the left panel of figure 11.

The model-dependence of the fragmentation function ratios seen by comparing the left and right panels of figure 11 is striking: the ordering of the predictions of the three models is opposite in the two panels, with the hybrid strongly coupled model predicting the least depression of the fragmentation function of the inclusive jets and the most depression of the fragmentation function of the associated jets. Seeing this motivates us to introduce a new observable in figure 12 in which we, in effect, take the ratio of the right panel of figure 11 to the left panel of figure 11. That is, we propose to compare the fragmentation function of inclusive (which is to say leading) jets to the fragmentation function of associated (lower energy) jets in $\mathrm{Pb}-\mathrm{Pb}$ dijet events. To avoid trivial kinematic differences between the energies of the inclusive and associated jets, we have constructed this ratio with jets in 


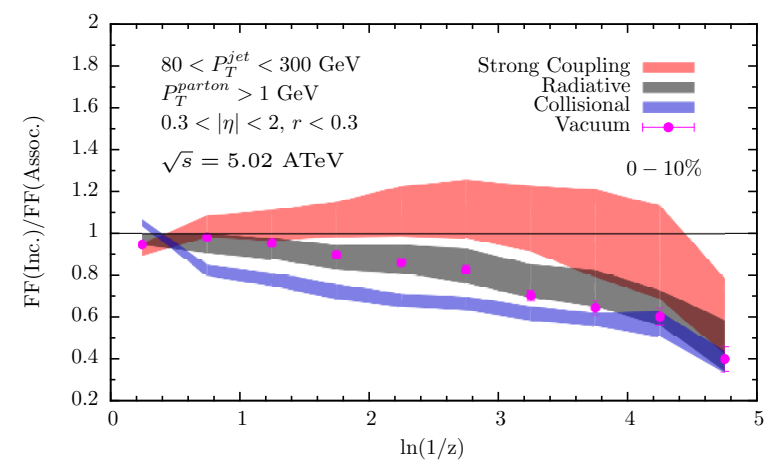

Figure 12. Model predictions for the ratio of the fragmentation functions of inclusive jets to the fragmentation functions of associated jets in dijet events whose most energetic jet has $p_{\mathrm{T}}>120 \mathrm{GeV}$ in $\mathrm{Pb}-\mathrm{Pb}$ collisions at $\sqrt{s}=5.02 \mathrm{ATeV}$. Both the inclusive jet and associated jet samples are constrained to the same $\eta$ and $p_{\mathrm{T}}$ range, see the text. We see a striking, and robust, separation between the predictions of our hybrid strongly coupled model and our two control models. We also show the same ratio, constructed with the same kinematic cuts, for inclusive and associated jets in p-p collisions. The ratio in p-p collisions should not be compared to that in $\mathrm{Pb}-\mathrm{Pb}$ collisions: each stands on its own and has its own implications. (Comparisons between $\mathrm{Pb}-\mathrm{Pb}$ and $\mathrm{p}-\mathrm{p}$ are shown in figure 11.) Here, the p-p results illustrate the differences between jets selected inclusively and jets selected by association with a leading jet in vacuum, with no jet quenching. And, the comparison between the $\mathrm{Pb}-\mathrm{Pb}$ results from the different models shows how the interplay between these differences and the path-length and energy dependence of different expressions for $d E / d x$ yields predictions for this ratio that depend sensitively on the underlying microscopic dynamics of jet quenching.

the same interval of $p_{\mathrm{T}}$ and rapidity, $80<p_{\mathrm{T}}<300 \mathrm{GeV}$ and $0.3<|\eta|<2$. As in figure 11, the associated jets whose fragmentation functions constitute the denominator in the ratio plotted in figure 12 are produced in association with leading jets of $p_{\mathrm{T}}>120 \mathrm{GeV}$. (We have not investigated other choices of leading jet $p_{\mathrm{T}}$.) The predictions of our hybrid strongly coupled model and of our two control models for this new observable are displayed in figure 12. This observable yields the largest separation between the predictions of our models of any observable that we have investigated. This means that it is particularly sensitive to the underlying microscopic dynamics behind the modification of jets in medium.

The new observable that we have introduced in figure 12 has the added virtue that its construction does not require fragmentation functions from jets in p-p collisions. It is the ratio of the fragmentation functions of differently selected $\mathrm{Pb}-\mathrm{Pb}$ jets with the same kinematics. This means that none of the uncertainties coming from the differences in the way hadronization occurs in $\mathrm{Pb}-\mathrm{Pb}$ collisions and $\mathrm{p}-\mathrm{p}$ collisions come into play. Our calculations behind figure 12 are, like those in section 4.1 and appendix B, calculations of partonic fragmentation functions. Here, though, we expect that the ratio of fragmentation functions displayed in figure 12 should be a better predictor for the same ratio of the hadronic fragmentation functions that experimentalists can measure than in the case of any of the other fragmentation function ratios that we have constructed because much of the uncertainty in our understanding of hadronization should cancel in this ratio. Of course, 
in the softest region, at the smallest $z$ 's, it continues to be the case that we are leaving out contributions to the fragmentation functions from the moving quark-gluon plasma, set in motion by the jet passing through it. This effect should at least partially cancel in the ratio plotted in figure 12; this is yet one more advantage of using the ratio of fragmentation functions of differently selected jets with the same kinematics in the same $\mathrm{Pb}-\mathrm{Pb}$ collisions, without introducing jets from p-p collisions.

In figure 12 we have also plotted (as purple dots) the same ratio of the fragmentation function of inclusive jets to that of associated jets for p-p collisions from PYTHIA, with the same kinematic cuts as in our $\mathrm{Pb}-\mathrm{Pb}$ analysis. We show this solely to confirm that even in the absence of any medium there is a difference between the inclusive jets and the associated jets. Selecting inclusive jets selects jets with fewer and therefore harder fragments. Selecting associated jets selects jets with more and therefore softer fragments. In $\mathrm{Pb}-\mathrm{Pb}$ collisions, then, in the presence of a medium the differences between the rates of energy loss come into play as we have discussed. This effect, plus the difference between the path lengths seen by inclusive and associate jets on average, are amplified by the different path-length and energy dependence of the rates of energy loss in our hybrid strongly coupled model and our two control models. The result is the large separation between the predictions of the models for the ratio plotted in figure 12, making this ratio so discriminating. We look forward to seeing experimental measurements of this ratio; they have great potential to teach us about the microscopic dynamics that results in the observed modification of jets in heavy ion collisions.

\section{Conclusions and outlook}

In this paper we have explored a broad range of jet observables in $\mathrm{Pb}-\mathrm{Pb}$ collisions at $\mathrm{LHC}$ energies within the context of the hybrid strong/weak coupling model that we introduced in ref. [23]. This is a phenomenological approach to the physics of jet quenching in which we aim to synthesize the very different types of physical processes involved in the production, branching, and subsequent in-medium dynamics of jets in heavy ion collisions. In particular, our model separates the short distance physics which controls the production and hard branching evolution of jets, which behaves as in vacuum, from the longer distance processes that control the interaction of the jet shower with the strongly coupled fluid produced in energetic heavy ion collisions. Our goal is to describe the short distance physics with standard tools for describing the weakly coupled physics of jets in vacuum, in particular with PYTHIA, and to describe the longer distance processes using insights from calculations done using strong coupling methods. In particular, to model the long distance physics we adopt results obtained by analyzing the rate of energy loss $d E / d x$ of an energetic massless parton propagating through the hot liquid plasma of strongly coupled $\mathcal{N}=4$ SYM theory, as obtained via gauge/gravity duality. We apply these results in QCD upon assuming that the differences between the hot liquid plasma phases of the two theories can be absorbed in a single parameter which controls the stopping distance of energetic excitations in plasma. We fitted this one model parameter to jet $R_{A A}$ data in our previous publication. Our model, now fully specified, yields a very good description of the suite of 
inclusive jet observables and dijet observables discussed in our previous publication and, as our explicit analysis in this paper shows, of all currently available data on photon-jet correlations. All these observables in sum span a wide range of energies, originate from different hard production processes with different primordial spectra, and correspond to jet samples with different selection biases, different ratios of quark jets to gluon jets, different fragmentation patterns and jet mass distributions, and that traverse different distributions of path lengths. Nevertheless, we have obtained a satisfactory description of all of these observables using our simple, hybrid, implementation of strongly coupled dynamics - namely by taking jets from PyтнiA and applying the strongly coupled rate of energy loss $d E / d x$ parton-by-parton to the partons in a parton shower.

Although at one level we are pleased by the now increasingly many successes of our hybrid model approach, at another level they are frustrating. Our model is simple, gluing together two rather different descriptions of physics at different scales and in so doing incorporating much unknown physics into just one parameter. Of course the first goal in creating such a model is to capture some of the physics correctly, and it seems that we have done that. However, it could be even more interesting to see the model breaking down and to use ways in which it fails to describe some feature of some experimental data to understand which of the aspects of the physics that the model leaves out are important, and how, and why, and where. The string of successes in the comparison between the results of our model calculations and experimental data preclude investigations of this nature at present.

All that said, our explicit analysis of two control models with parametrically different expressions for $d E / d x$ cautions us that we should not rush to conclude from the successes of our hybrid model that the experimental measurements of the various jet, dijet and photonjet observables favor the strongly coupled form for $d E / d x$ over other possibilities. Rather, we must acknowledge that these observables are not strongly sensitive to the parametric form of the rate of energy loss $d E / d x$. The experience that we have gained from our analysis suggests that as long as the vacuum-like branching processes that are at the core of jet dynamics are described well, any mechanism that is able to quench particles, in particular the softer partons in a jet, can capture the bulk features of the measured distributions of jet observables, including the various dijet and photon-jet asymmetries and correlations, as long as the parameter that governs the overall magnitude of $d E / d x$ is chosen appropriately.

Nevertheless, it is certainly heartening and perhaps even remarkable that the range of values of the one parameter in our hybrid strong/weak coupling model, $\kappa_{\mathrm{sc}}$, that we find provides a good description of so much jet data agrees so well with a priori expectations. $\kappa_{\mathrm{sc}}$ should be smaller than but of order one, exactly as we have obtained. A stopping length for energetic partons in the strongly coupled QGP of QCD that is three to four times longer than that in the strongly coupled $\mathcal{N}=4 \mathrm{SYM}$ plasma with the same temperature, as we find, is an eminently reasonable result.

These conclusions are all conclusions that we reached in our previous publication. The analyses of photon-jet observables presented here serves to reinforce them in many ways.

Looking ahead, we hope that among the many calculations from our hybrid model that we have presented the ones that will be most important will be the many predictions 
that we are making for experimental measurements that are anticipated in the near future. These can be grouped into three categories, the first two of which are:

- We have provided the predictions of our hybrid model for inclusive jet observables, dijet observables, and photon-jet observables in heavy ion collisions with collision energy $\sqrt{s}=5.02 \mathrm{ATeV}$, in anticipation of LHC heavy ion Run 2. This is particularly important for the photon-jet observables. The present data have low statistics, and correspondingly large error bars, making the fact that our hybrid model describes them well less impressive than it could be. In the run to come, the statistics will be greater by about an order of magnitude, meaning that the error bars should be significantly smaller, making the confrontation between the predictions of our model and these measurements much more constraining.

- We have provided the predictions of our hybrid model for Z-jet observables, which we hope will be tested in future LHC runs.

Confrontation between these predictions and the data to come should serve either to further strengthen our confidence in the approach to jet quenching that we have introduced or to identify and quantify ways in which it fails, ideally pointing toward which aspects of the physics that we have left out are most important and guiding the improvement of the model. It is also possible that as the experimental uncertainties shrink measurements of these observables could serve to differentiate between the different assumptions about the dynamics of parton energy loss, and consequent different forms for $d E / d x$. However, the distinctions between the predictions of our hybrid model and our two control models for these observables are small, limiting the discriminating power of these observables even as the experimental uncertainties shrink.

It seems clear that in order to find observables that provide more discrimination among the different possible dynamical processes via which the partons in a shower lose energy as they traverse the strongly coupled plasma we will need to investigate intrajet observables. Utilizing those observables that involve the angular shape of jets must wait, as it will require adding further physics to the model, including for example the transverse momentum picked up by the shower partons as they interact with the medium, and so must involve the addition of at least one new parameter to the model. There is good motivation for such investigations, but we leave them to future work. With the one parameter model that we have constructed here, the class of intrajet observables that we may be able to describe is those constructed from fragmentation functions:

- The third category of predictions that we have made are predictions for various ratios of fragmentation functions. We have taken advantage of having a tool with which we can compute multiple observables (here, fragmentation functions for jets in p-p collisions, inclusive jets and jets in dijet pairs in $\mathrm{Pb}-\mathrm{Pb}$ collisions, and jets produced in association with photons or Z-bosons in either $\mathrm{Pb}-\mathrm{Pb}$ or $\mathrm{p}$-p collisions) in varying kinematic regimes to search for new discriminating observables which are particularly sensitive to differences between mechanisms of energy loss. We have provided the results of our hybrid model calculations for ratios between partonic fragmentation 
functions in photon-jet and Z-jet events in $\mathrm{Pb}-\mathrm{Pb}$ collisions to those in p-p collisions, but for reasons that we have understood qualitatively these turn out not to be particularly discriminating. Also, these predictions are sensitive to differences between hadronization dynamics in $\mathrm{Pb}-\mathrm{Pb}$ and $\mathrm{p}-\mathrm{p}$ collisions that are not under good theoretical control at present, reducing the reliability of our predictions for these ratios. The most discriminating observable that we have found is the ratio of the fragmentation function of inclusive jets in $\mathrm{Pb}-\mathrm{Pb}$ collisions to the fragmentation functions of jets in the same kinematic regime, in the same collisions, that are the lower energy jets in a dijet pair. (We refer to the latter as associated jets.) Unlike most jet observables, this measurement does not require any p-p reference data. And, since the ratio we propose is constructed from jets in the same kinematic regime in the same $\mathrm{PbPb}$ collisions, differing only in how they were selected, we expect that many of the theoretical uncertainties associated with the modification of hadronization in medium should cancel. This makes the ratio of partonic fragmentation functions that we compute a better proxy to the ratio of fragmentation functions that experimentalists will measure. Furthermore, we find that the predictions of our hybrid strong/weak coupling model and our two control models for this ratio, see figure 12, are well separated over a wide range of $z$, making the discriminating power of this observable robust even after the softening of the fragmentation functions expected after hadronization. And, perhaps best of all, we have a good qualitative understanding of why this ratio is such a discriminating observable. First, in $\mathrm{Pb}-\mathrm{Pb}$ collisions as in vacuum inclusive jets tend to contain fewer, harder, fragments than associated jets on average. Second, the distribution of the path length of the medium through which a sample of inclusive jets has propagated is, on average, shorter than that for a sample of associated jets. Both these effects mean that, on average, the inclusive jets have lost less energy than the associated jets. And, we have shown that both these effects push the predictions of our hybrid strong/weak coupling model and our control models apart. Measurement of this ratio of fragmentation functions should be a particularly effective way to gain information about the dynamics via which energetic partons lose energy as they traverse strongly coupled plasma. The experimental determination of this ratio in the imminent LHC heavy ion Run 2 can therefore shed light on the microscopic dynamics of jets in quark-gluon plasma.

\section{Acknowledgments}

We gratefully acknowledge the assistance of Chun Shen and Ulrich Heinz, who have generously provided us with hydrodynamic solutions for heavy ion collisions at $\sqrt{s}=2.76$ and 5.02 ATeV. We are grateful to Aaron Angerami, Paul Chesler, Yen-Jie Lee, Gunther Roland, Konrad Tywoniuk, Wilke van der Schee and Xin-Nian Wang for helpful conversations over the course of this work. KR is grateful to the CERN Theory Division for hospitality at the time this research was completed. The work of JCS was supported by a Ramón y Cajal fellowship. The work of JCS and DP was supported by the Marie Curie Career Integration Grant FP7-PEOPLE-2012-GIG-333786, by grants FPA2013-46570 and 
FPA2013-40360-ERC and MDM-2014-0369 of ICCUB (Unidad de Excelencia 'María de Maeztu') from the Spanish MINECO, by grant 2014-SGR-104 from the Generalitat de Catalunya and by the Consolider CPAN project. The work of DCG and KR was supported by the U.S. Department of Energy under Contract Numbers DE-SC0011088 and DE-SC0011090, respectively. The work of JGM was supported by Fundação para a Ciência e a Tecnologia (Portugal) under project CERN/FP/123596/2011 and contract 'Investigador FCT - Development Grant'.

\section{A Energy Loss in a boosted fluid}

In this appendix, we explicitly perform the Lorentz transformation that, as we explained in section 2.2 , is needed in order to determine the rate of energy loss $d E / d x$ in the collision center-of-mass frame (the frame in which we do the overall computation of the modifications to the energies of the partons in the shower) from the rate of energy loss in the local fluid rest frame (the frame in which the fluid at the spacetime location of a particular parton is at rest). This transformation is particularly important for partons with significant rapidity, as they propagate through fluid that is moving with a significant velocity, meaning that the boost from the local fluid rest frame back to the collision center-of-mass frame is substantial.

In the local fluid rest frame, the change in the four-momentum of a parton which propagates for an infinitesimal time $d t_{F}$ is given by

$$
d P_{F}^{\mu}=\mathcal{F}_{F}\left(x_{F}, E_{\text {in }}^{F}\right) \frac{P_{F}^{\mu}}{E_{F}} d t_{F},
$$

where and $P_{F}^{\mu}$ and $E_{F}$ are the four momentum and energy of the parton in the local fluid rest frame and $\mathcal{F}_{F}\left(x, E_{\text {in }}^{F}\right)$ is the functional form of the rate of energy loss in that frame, in the notation that we introduced in eq. (2.3), and is given by the right-hand side of (2.1) in our hybrid model or by the right-hand side of one of the expressions (2.2) in our control models. In writing the expression (A.1) we have used our assumption that the exchanges of momentum and energy between the parton and the medium do not change the direction of the parton significantly.

The Lorentz structure of the expression (A.1) simplifies the boost back to the collision center-of-mass frame. First of all, it is easy to show that

$$
\frac{d t_{F}}{E_{F}}=\frac{d t}{E}
$$

where $t$ and $E$ are the time and energy in the collision center-of-mass frame. Second, after a Lorentz transformation, $P_{F}^{\mu} \rightarrow P^{\mu}$ and $d P_{F}^{\mu} \rightarrow d P^{\mu}$, with $P^{\mu}$ and $d P^{\mu}$ the four-momentum and the infinitesimal four-momentum loss in the collision center-of-mass frame. Therefore

$$
\frac{d P^{\mu}}{d t_{F}}=\mathcal{F}_{F}\left(x_{F}, E_{\text {in }}^{F}\right) \frac{P^{\mu}}{E},
$$

where the arguments of $\mathcal{F}_{F}$ are still expressed in terms of quantities in the local fluid rest frame. 
We next express the initial parton $E_{\text {in }}^{F}$ in the local fluid rest frame as a function of quantities in the collision center-of-mass frame via the Lorentz transformation

$$
E_{\text {in }}^{F}=E_{\text {in }} \gamma_{F}(1-\mathbf{w v}),
$$

where $\mathbf{w}=\mathbf{P} / E$ is the velocity of the parton in the collision center-of-mass frame and $\mathbf{v}$ is the local velocity of the fluid in the same frame, which is to say it is the velocity vector for the boost between the two relevant frames. $\gamma_{F}$ is the gamma factor of the local fluid velocity $\mathbf{v}$.

The relation between $x_{F}$ and $x$, the distances travelled in the two frames, requires further discussion. In the derivation of the rate of energy loss by one of the partons in the shower, $x_{F}$ is the distance that that parton has travelled through the fluid. However, as partons propagate through the hot plasma created in an heavy ion collision, the temperature and velocity of the fluid at their location in space and time changes. We will assume that $x_{F}$ is the accumulated distance of the parton summed in such a way that each infinitesimal contribution $d x_{F}$ is evaluated in the local fluid rest frame. This means that (if the rate of energy loss depends on $x_{F}$, as in (2.1) and the first expression in (2.2)) the energy lost by a parton traversing some $d x_{F}$ depends on the total $x_{F}$ accumulated by that parton over its previous passage through the flowing plasma. With this prescription, which neglects gradient effects, we have

$$
d \mathbf{x}_{F}=\mathbf{w} d t+\gamma_{F}\left(\mathbf{w}_{L}-\mathbf{v}\right) d t
$$

where $\mathbf{w}_{T, L}$ are the transverse and longitudinal components of the parton velocity in the collision center-of-mass frame. After some algebra, the increment in the accumulated distance is given by

$$
\left(\frac{d x_{F}}{d t}\right)^{2}=\mathbf{w}^{2}+\gamma_{F}^{2}\left(\mathbf{v}^{2}-2 \mathbf{v w}+(\mathbf{v w})^{2}\right)
$$

Summing over the previous history of the parton, we obtain

$$
x_{F}(t)=\int_{t_{0}}^{t} d t \sqrt{\left[\mathbf{w}^{2}+\gamma_{F}^{2}\left(\mathbf{v}^{2}-2 \mathbf{v w}+(\mathbf{v w})^{2}\right)\right]},
$$

where $t_{0}$ is the creation time of the parton. We have used (A.3), (A.4) and (A.7) in (2.5), (2.6) and (2.7).

\section{B Update on single-jet and dijet observables at $\sqrt{s}=2.76 \mathrm{ATeV}$}

In this appendix, we update our analysis of the single-jet and dijet observables that we presented in ref. [23] to include the effect of fluid flow on the rate of energy loss, as described in section 2.2. We also employ the viscous hydrodynamic simulations of ref. [87], as described in section 3.1 and as in all results presented in this paper. As discussed in section 2.3, we have followed the same fitting procedure as we used in ref. [23] to determine the value of the single parameter which controls each the rate of energy loss in our hybrid 

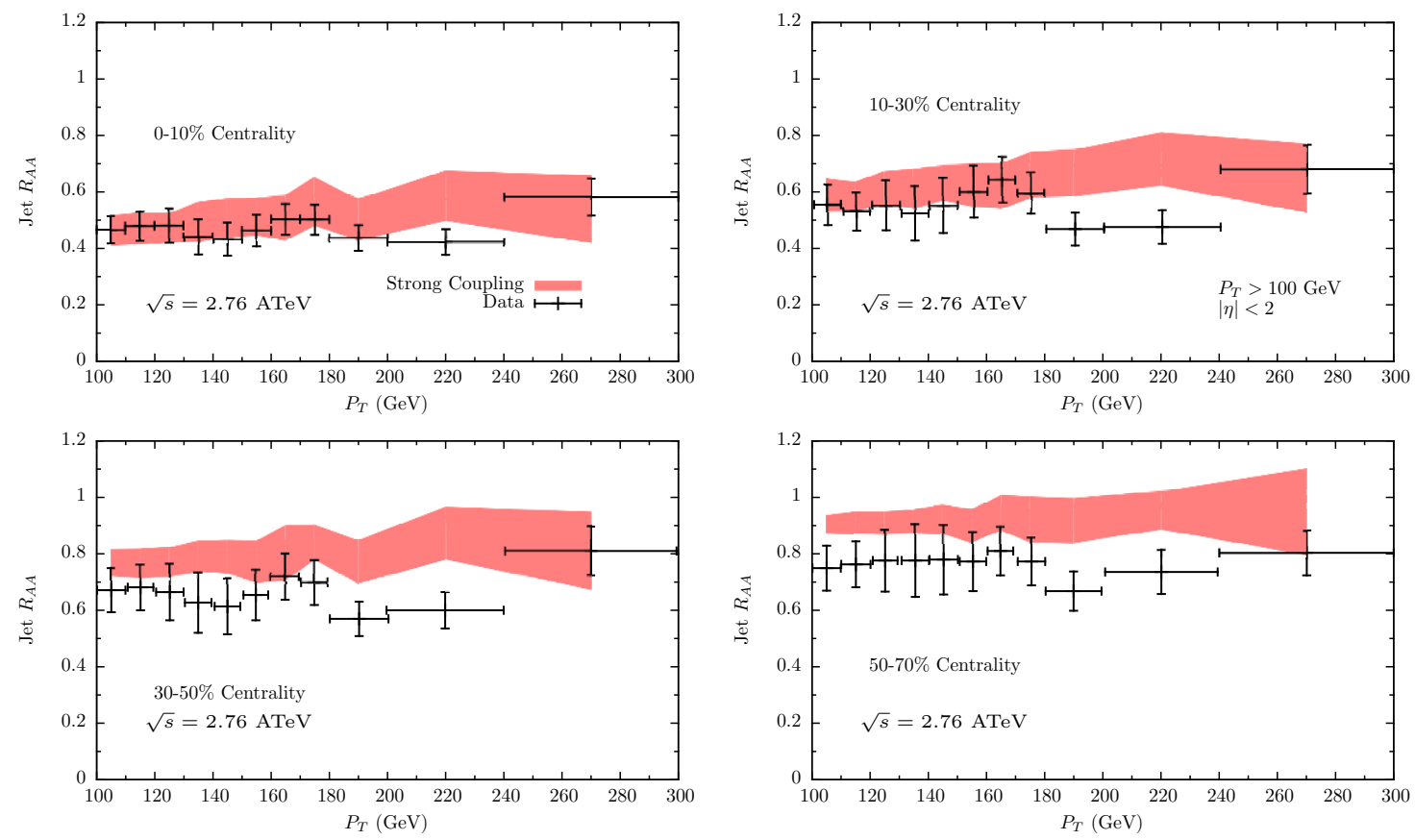

Figure 13. Jet $R_{\mathrm{AA}}$ as a function of $p_{\mathrm{T}}$ for different centralities from our hybrid strong/weak coupling model (colored bands) compared to preliminary CMS data from ref. [103]. The value of the single parameter in the model, $\kappa_{\mathrm{sc}}$, is fitted to the left-most data point in the top-left panel, namely the jets with $100 \mathrm{GeV}<p_{\mathrm{T}}<110 \mathrm{GeV}$ in the most central collisions. All the rest of the features of the colored bands are results from our hybrid model. In this appendix and throughout this paper, single-jet, dijet, photon-jet, Z-jet and fragmentation function observables are all fully specified once the single parameter in the model has been fixed.
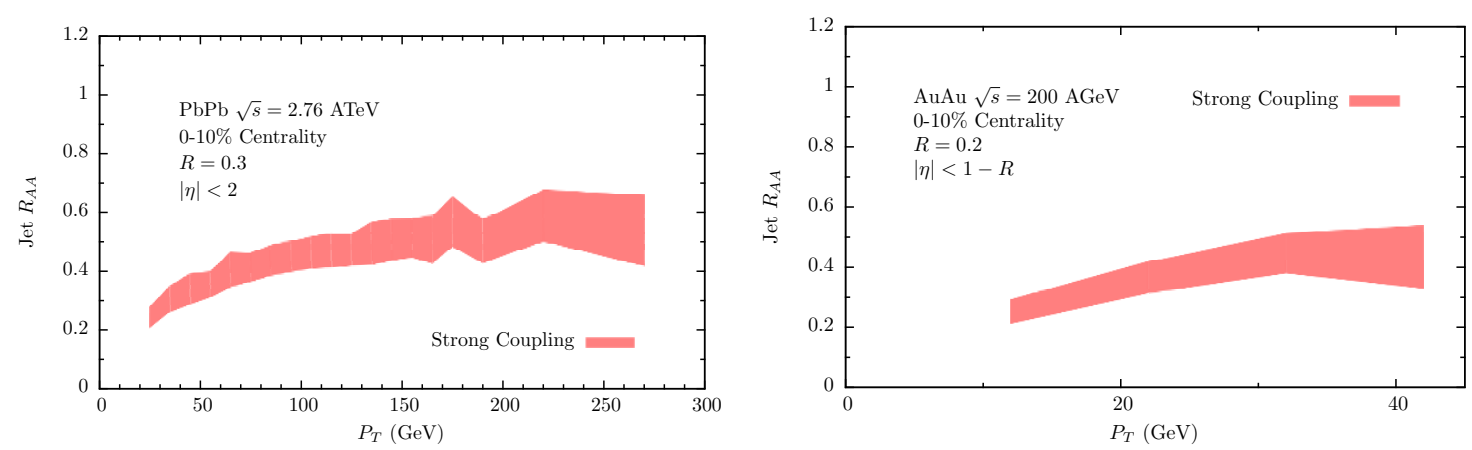

Figure 14. Predictions of our hybrid strongly coupled model for jet $R_{\mathrm{AA}}$ for central $\mathrm{Pb}-\mathrm{Pb}$ collisions at the LHC with $\sqrt{s}=2.76 \mathrm{ATeV}$ as a function of $p_{\mathrm{T}}$, extended down to $p_{\mathrm{T}}=15 \mathrm{GeV}$ (left) and for central Au-Au collisions at RHIC with $\sqrt{s}=200 \mathrm{AGeV}$ (right). In both cases, we only show our results for collisions in the $0-10 \%$ centrality bin.

model and in our two control models. The results of these fits are summarized in table 1. Since the change in all the observables is minor, in this appendix we will not describe each observable in detail. We simply plot the results in figures 13, 14, 15, 16 and 17, and refer the reader to the our previous publication for all discussion. 

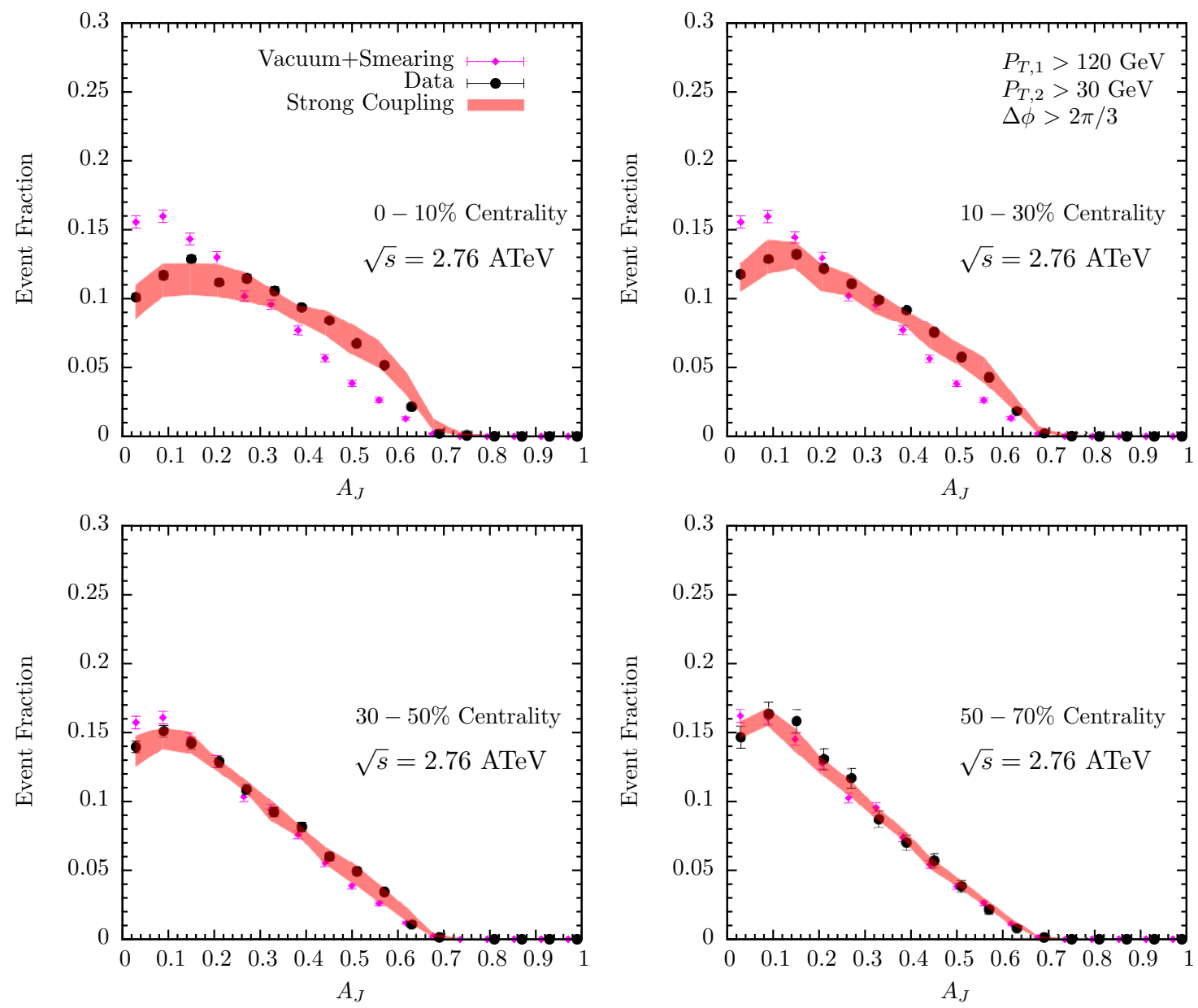

Figure 15. Dijet imbalance $A_{J}$ in heavy ion collisions with $\sqrt{s}=2.76 \mathrm{ATeV}$ from our hybrid model (colored bands) compared to CMS data from ref. [7] (black) and PYTHIA-generated proton-proton collisions (violet). Both the hybrid model calculations and the proton-proton reference are smeared according to the prescription in ref. [114].

\section{Predictions for single-jet and dijet observables at $\sqrt{s}=5.02 \mathrm{ATeV}$}

In this appendix, we provide predictions for the single jet and dijet observables that we presented in detail in our previous publication [23] and updated in appendix $\mathrm{B}$, now for $\mathrm{Pb}-\mathrm{Pb}$ collisions at $\sqrt{s}=5.02 \mathrm{ATeV}$. We study $10^{6}$ dijet events in p-p collisions at $\sqrt{s}=5.02 \mathrm{TeV}$ generated by PYтнIA 8.183 [86] without any underlying event. For each of the centrality bins we consider, we embed these hard scattering processes into the hydrodynamic simulations along the lines of those in ref. [87] as we described in section 2.3, but simulations of heavy ion collisions with $\sqrt{s}=5.02 \mathrm{ATeV}$. We then follow the procedure for determining the energy loss outlined in section 3.1. As in our previous computations, we smear our predictions to simulate resolution effects. However, and similarly to the procedure we have followed in obtaining our photon-jet and Z-jet predictions described in sections 3.2 and 3.3, we use the smearing functions determined at $\sqrt{s}=2.76 \mathrm{ATeV}$, since they are yet unknown at $\sqrt{s}=5.02 \mathrm{ATeV}$. 

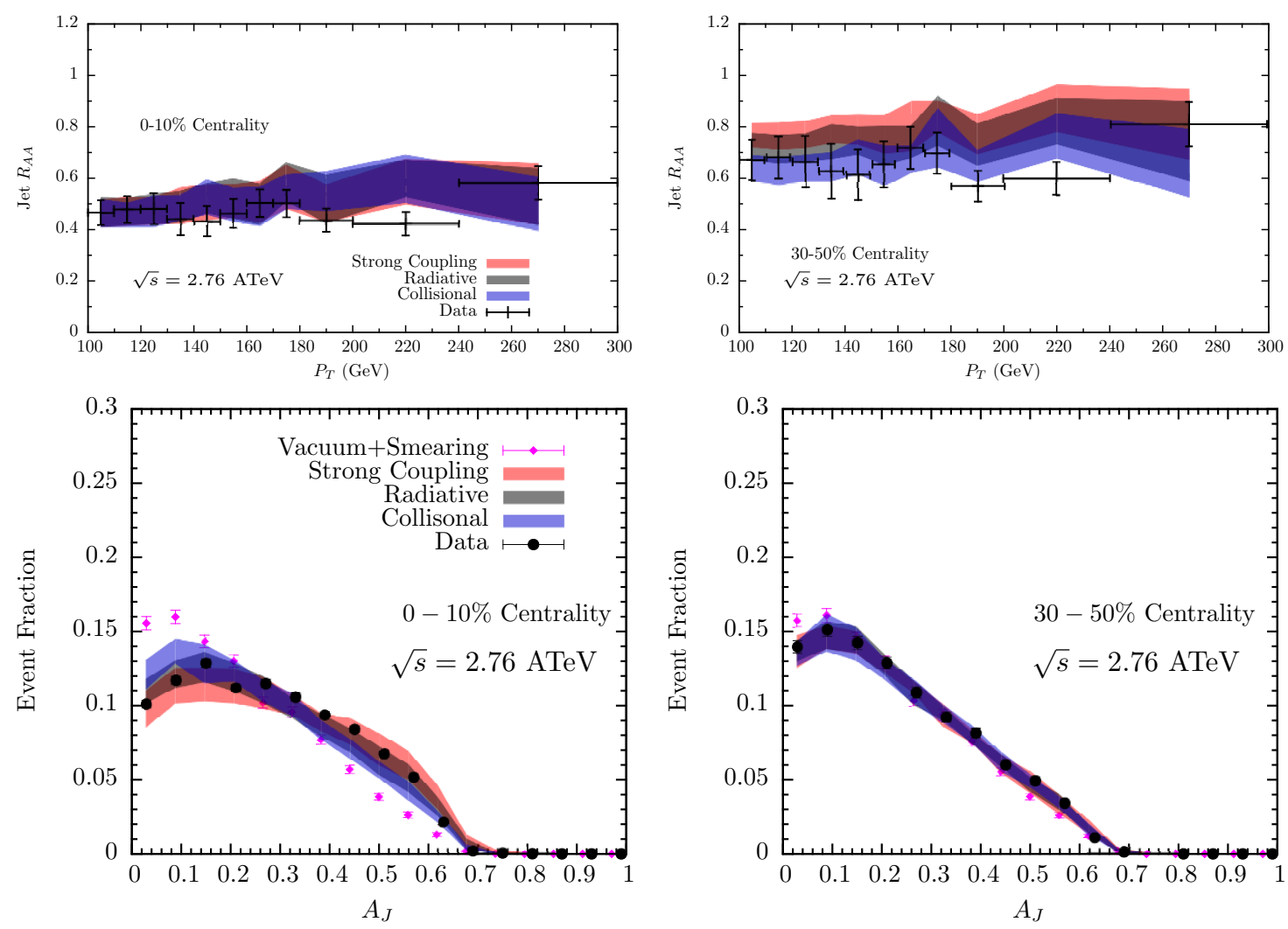

Figure 16. Upper panel: jet $R_{\mathrm{AA}}$ as a function of $p_{\mathrm{T}}$ for heavy ion collisions with $\sqrt{s}=2.76 \mathrm{ATeV}$ in two different centrality bins for the hybrid model with its strongly coupled rate of energy loss and the two control models from section 2.1, as compared to preliminary CMS data [103]. Lower panel: dijet imbalance distribution in two different centrality bins for those three energy loss models, as compared to CMS data from ref. [7]. Each of the three models for the rate of energy loss $d E / d x$ includes one free parameter, and in each case we have fitted the value of this parameter to obtain agreement between the model and the data for $100 \mathrm{GeV}<p_{\mathrm{T}}<110 \mathrm{GeV}$ in the most central $(0-10 \%)$ collisions.

Figure 18 shows the hybrid strong/weak coupling model predictions for the suppression factor $R_{A A}^{\text {jet }}$ of jets in heavy ion collisions with $\sqrt{s}=5.02 \mathrm{ATeV}$ reconstructed with the anti$k_{T}$ algorithm with $R=0.3$. We do not fit even a single parameter here, or anywhere in this appendix. The model is fully constrained by the data on collisions with $\sqrt{s}=2.76 \mathrm{ATeV}$, with its one parameter having been fixed as described in section 2.3 and appendix B. The width of the displayed bands is a combination of our theoretical uncertainties (estimated by varying the temperature $T_{c}$ below which we stop quenching, see section 2) together with the experimental uncertainties in the data that we use to fix the one parameter in each model. Both the centrality dependence and the transverse momentum dependence of $R_{A A}^{\mathrm{jet}}$ in collisions with $\sqrt{s}=5.02 \mathrm{ATeV}$ are very similar to what we have seen previously at $\sqrt{s}=2.76 \mathrm{ATeV}$, with a slight increase in the suppression of $R_{A A}^{\text {jet }}$ at the higher collision energy. Similar conclusions can be drawn from the strong coupling predictions for the dijet imbalance in collisions with $\sqrt{s}=5.02 \mathrm{ATeV}$, displayed in figure 19. The centrality 

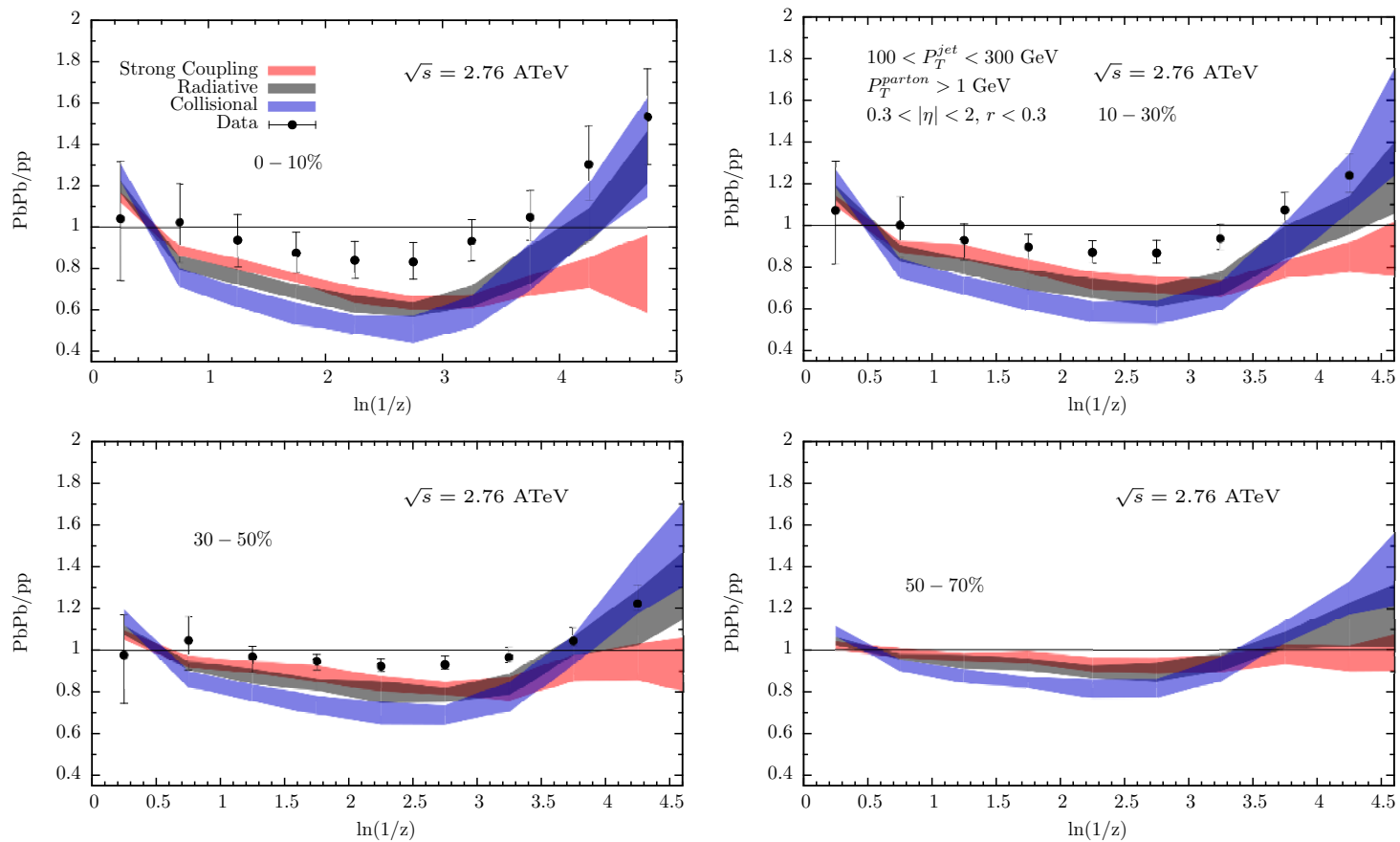

Figure 17. Partonic fragmentation function ratios (fragmentation function for jets in $\mathrm{Pb}-\mathrm{Pb}$ collisions over that for jets in p-p collisions) for jets with $100<p_{\mathrm{T}}{ }^{\text {jet }}<300 \mathrm{GeV}$ in heavy ion collisions with $\sqrt{s}=2.76 \mathrm{ATeV}$ with four different centralities from the hybrid model and the two control models of the rate of energy loss. Jets are reconstructed with the anti- $k_{T}$ algorithm with $R=0.3$. The jet fragments consist of final state partons within a cone of radius $r=0.3$ around the jet axes determined by the reconstruction algorithm. These partons are classified with respect to the longitudinal variable $z=p^{\|} / p^{\text {jet }}$ with $p^{\|}$the component of the momentum of the fragment along the jet axis. Note that in the softest region of the fragmentation function, say $\ln (1 / z) \gtrsim 3.5-4$, there is an additional contribution to the fragmentation function that is not included in the model: as the jet deposits energy and momentum into the medium, some part of the medium ends up moving as a wake in the direction of the jet. This will serve to push up the softest region of the fragmentation function in all our models, by an amount that should be model independent to a good approximation. This effect is not included in our models, but does contribute in the experimental data. The three models are compared to the data from ref. [15]. The separation between the models is cause for optimism that the higher statistics measurements expected from LHC heavy ion Run 2 may serve to distinguish between models. This optimism must be tempered, however, given that the predictions of the models do not differ in a qualitative way and given that we have left out both hadronization and the response of the medium to the passage of the jet.

dependence and the momentum dependence of this observable are very similar at the two collision energies as well.

The sensitivity of these predictions to the form we assume for the rate of energy loss is examined in figure 20. As at the lower collision energy, this set of predictions shows little discriminating power to our choice among the three models for the rate of energy loss that we have investigated. The strongly coupled form (2.1) for $d E / d x$ leads to a slightly large suppression and a slightly bigger dijet imbalance than the two control models (2.2), bottom-left panel of figure 20, but the effect is small compared to current uncertainties. 

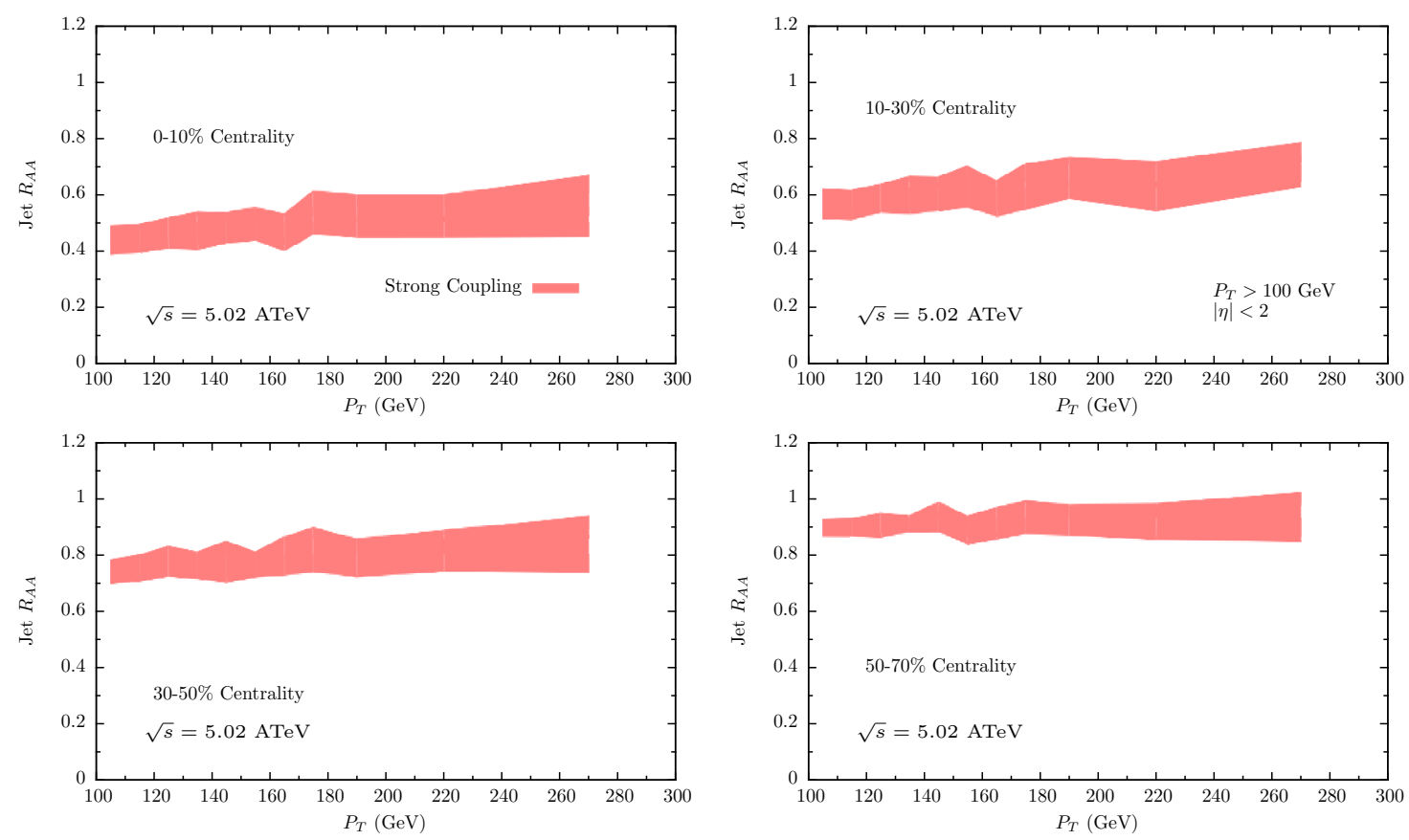

Figure 18. Hybrid model prediction for Jet $R_{\mathrm{AA}}$ as a function of $p_{\mathrm{T}}$ for different centralities at $\sqrt{s}=5.02 \mathrm{ATeV}$. The single model parameter has been fitted to $\sqrt{s}=2.76 \mathrm{ATeV}$ data previously and no additional parameters have been introduced.

Some separation among models is observed with increasing centrality, top right panel of figure 20, although the largest centralities are more and more sensitive to the energy lost by energetic partons during the hadronic phase of the collision, which we are neglecting.

Finally, in figure 21 we compare the predictions for partonic fragmentation functions obtained from the three models for the rate of energy loss. These are, again, similar to one another. The modest separation between the model predictions at intermediate values of $z$ observed at $\sqrt{s}=2.76 \mathrm{ATeV}$ is also observed at $\sqrt{s}=5.02 \mathrm{ATeV}$. However, the model predictions separate most in the region of $\log z>3.5$ where they cannot be relied upon. In this softest region of the fragmentation function, there is an additional contribution that is not included in the model: the backreaction of the medium to the jet passing through it will result in additional soft particles in the jet cone.

\section{Model dependence of boson-jet correlations}

In this appendix, we study the sensitivity of the different photon-jet and Z-jet observables that we have considered in sections 3.2 and 3.3 to the microscopic mechanism responsible for energy loss. To do so, we repeat our analysis with the strongly coupled form for the rate of energy loss (2.1) that we use in our hybrid model replaced by one or other of the expressions (2.2) that define our two control models, which are inspired by the radiative and collisional energy loss mechanisms. As in the strong coupling case, these expressions for $d E / d x$ also contain a single parameter which is fitted to the single-jet production rate 

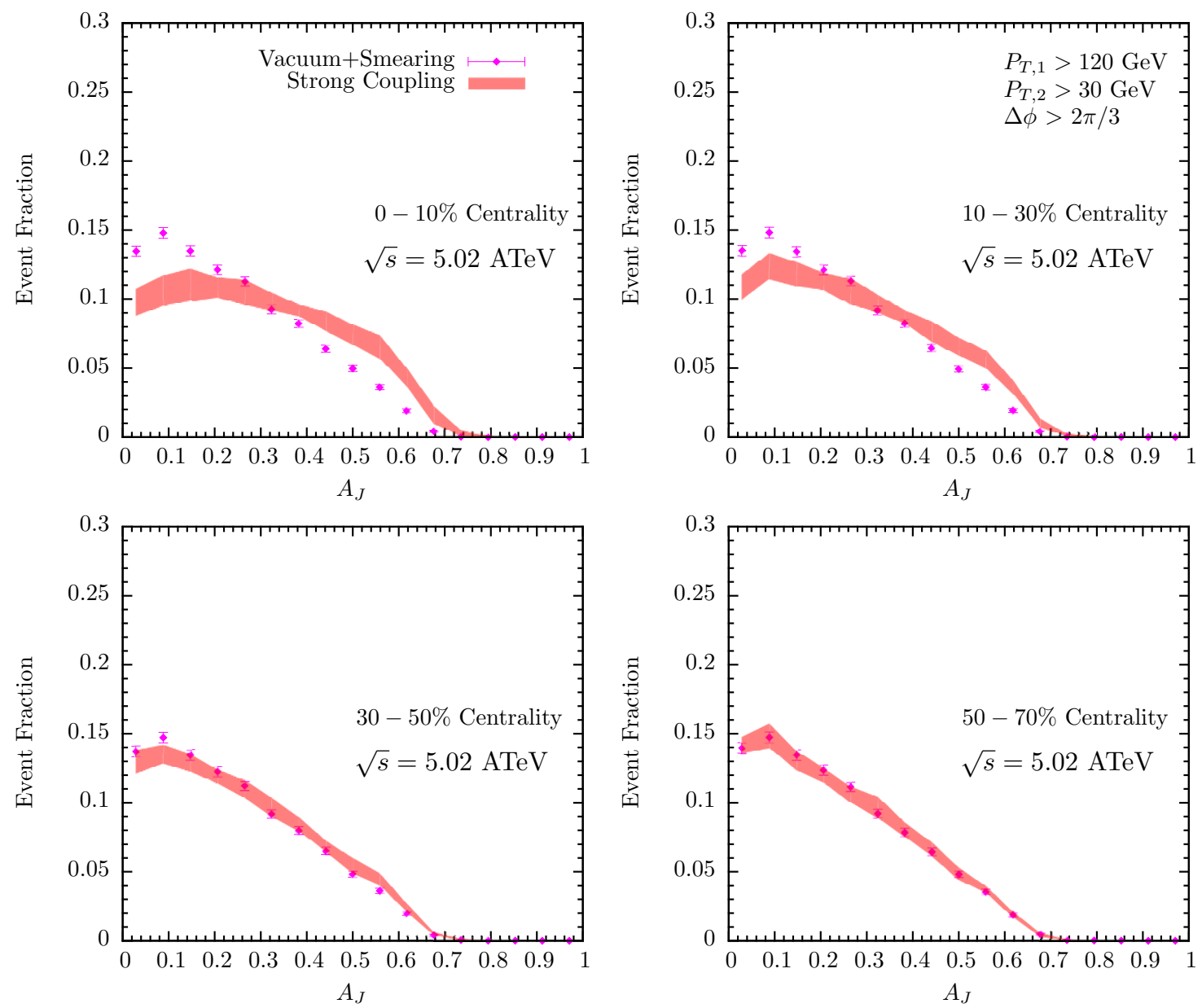

Figure 19. Hybrid model predictions for the dijet imbalance $A_{J}$ in $\mathrm{Pb}-\mathrm{Pb}$ collisions at $\sqrt{s}=5.02 \mathrm{ATeV}$ with different centralities. Both the theoretical calculations and the proton-proton reference are smeared according to the prescription in ref. [114].

at one transverse momentum and centrality. The values of the parameters obtained from these fits are summarized in table 1.

In figure 22 we show the results that we have obtained from the hybrid strong/weak coupling model and our two control models for the photon-jet observables that we have analyzed in heavy ion collisions with $\sqrt{s}=2.76 \mathrm{ATeV}$. The procedure for determining each of the observables is identical for all models and is described in section 3.2. These plots make clear that the uncertainties in the present low-statistics data are too large to make it possible to use the photon jet imbalance or the spectrum of jets produced in association with isolated photons or the fraction of isolated photons with an associated jet to differentiate between microscopic models of the dynamics of energy loss. Within the current uncertainties, all the models agree with the CMS data displayed in these plots. The photon-jet imbalance in central collisions, top left panel of figure 22, does have modest power to discriminate between the strongly coupled model and the control models. This indicates that the higher statistics photon-jet data sets anticipated in LHC heavy ion Run 

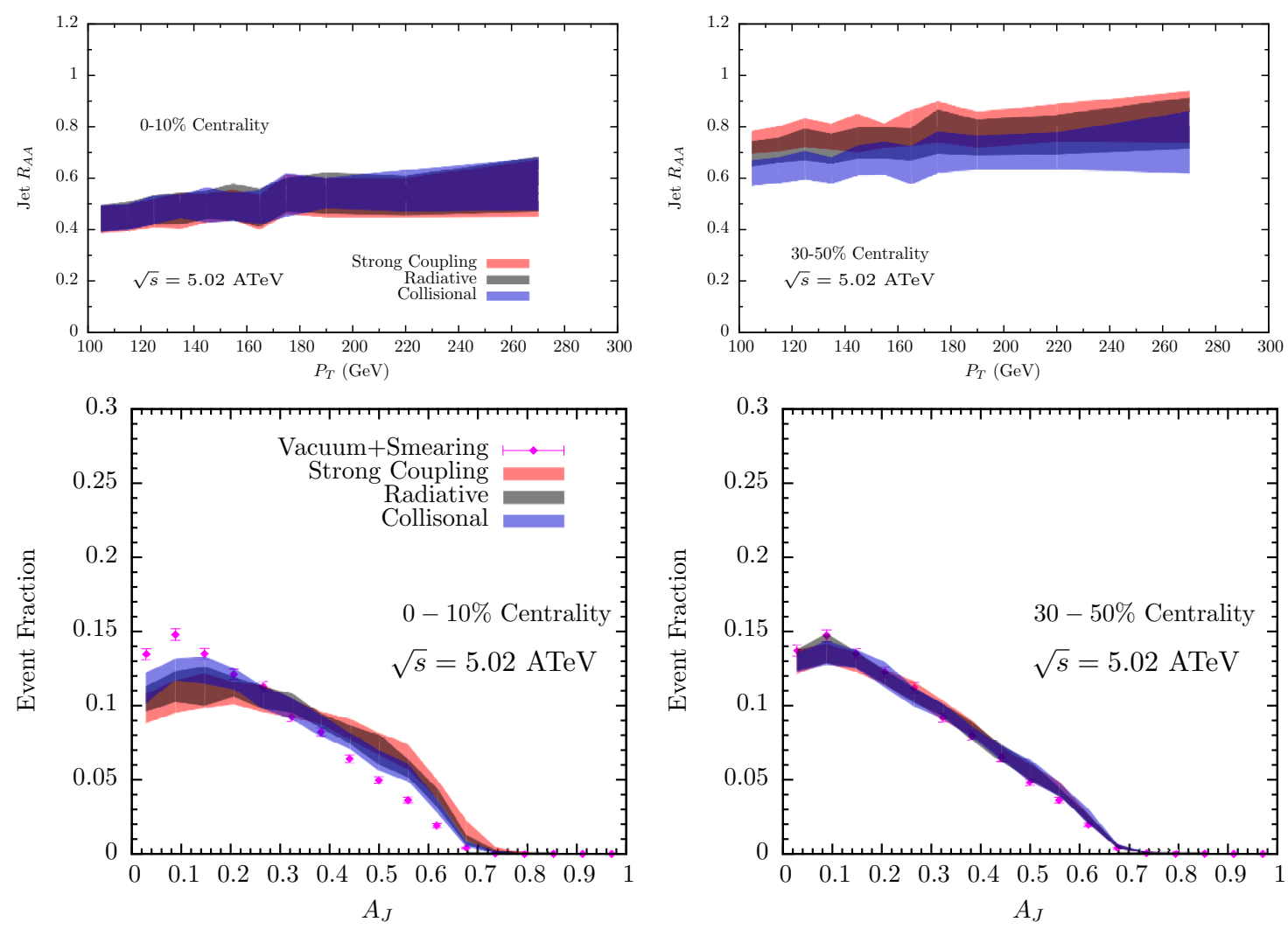

Figure 20. Model dependence of dijet observables for $\mathrm{Pb}-\mathrm{Pb}$ collisions with $\sqrt{s}=5.02 \mathrm{ATeV}$. Upper panel: jet $R_{\mathrm{AA}}$ as a function of $p_{\mathrm{T}}$ for LHC collisions in two different centrality bins for the three energy loss models from section 2.1. Lower panel: dijet imbalance distribution in two different centrality bins for the three energy loss models.

2 could shed light on the microscopic dynamics via which jets lose energy, as well as on the quantitative validity of the simplifying assumptions inherent in our hybrid model.

To better compare our computations with future higher statistics data from LHC heavy ion Run 2, we also explore the model predictions of the different energy loss mechanisms for both photon-jet observables, displayed in figure 23, and Z-jet observables, displayed in figure 24, in heavy ion collisions with $\sqrt{s}=5.02 \mathrm{ATeV}$. As at the lower collision energy, little discriminating power is observed. Again as at the lower collision energy, there is some separation among the predictions of our hybrid model with its strongly coupled rate of energy loss and the control models in the photon-jet and Z-jet momentum imbalance distributions in the most central collisions, displayed in the upper-left panels of figures 23 and 24 , respectively. 

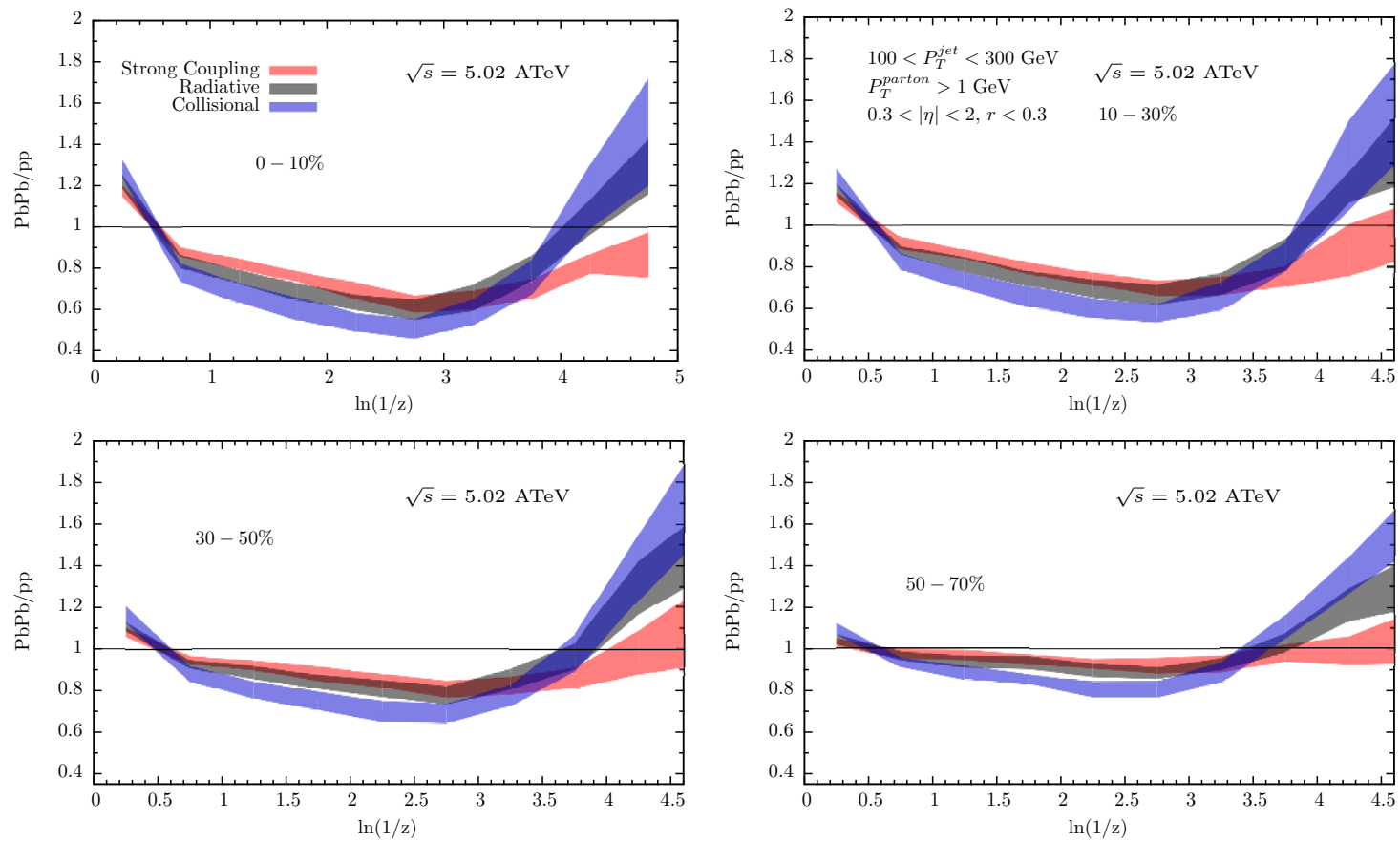

Figure 21. Partonic fragmentation functions for jets of $100<p_{\mathrm{T}}^{\text {jet }}<300 \mathrm{GeV}$ in heavy ion collisions with $\sqrt{s}=5.02 \mathrm{ATeV}$ for three different models for the rate of energy loss and for four different centralities. Jets are reconstructed with the anti- $k_{T}$ algorithm with $R=0.3$. The jet fragments consist of final state partons within a cone of angle $r=0.3$ around the jet axes determined by the reconstruction algorithm. These partons are classified with respect to the longitudinal variable $z=p^{\|} / p^{\text {jet }}$ with $p^{\|}$the momentum of the fragments along the jet axis. 

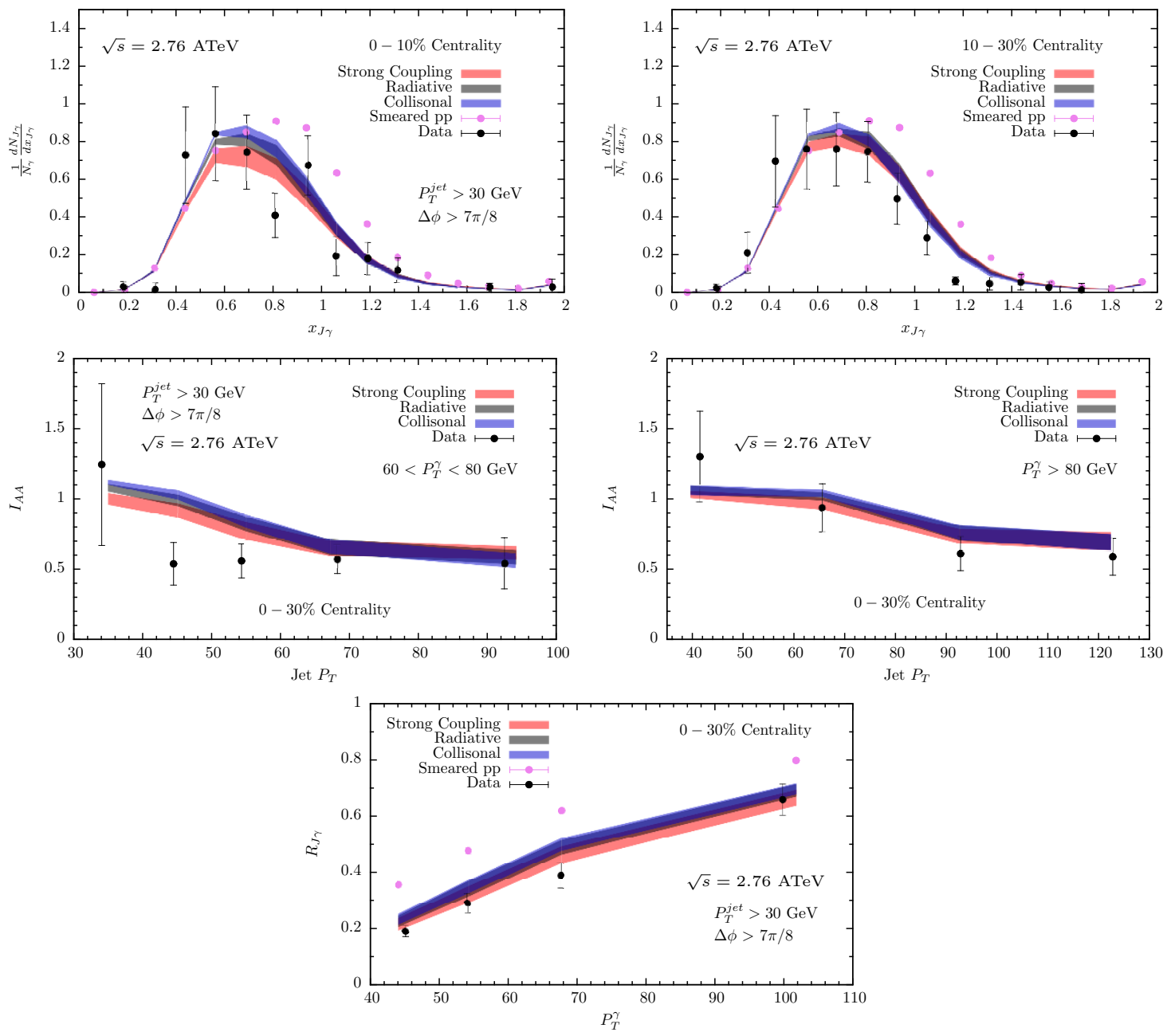

Figure 22. Computations of several photon-jet observables using three different models of the energy loss mechanism for heavy ion collisions with $\sqrt{s}=2.76 \mathrm{ATeV}$. The distributions of the transverse momentum imbalance of photon-jet pairs for two different centralities are displayed in the upper panels. The middle panel shows the ratio of the transverse momentum spectra of jets produced in association with an isolated photon in $\mathrm{Pb}-\mathrm{Pb}$ collisions to that in $\mathrm{p}-\mathrm{p}$ collisions for two different centralities. The lower panel shows the fraction of isolated photons produced in association with a hard jet with $p_{\mathrm{T}}{ }^{\text {jet }}>30 \mathrm{GeV}$ at an azimuthal angle more than $7 \pi / 8$ away from that of the isolated photon. Data are taken from ref. [111]. 

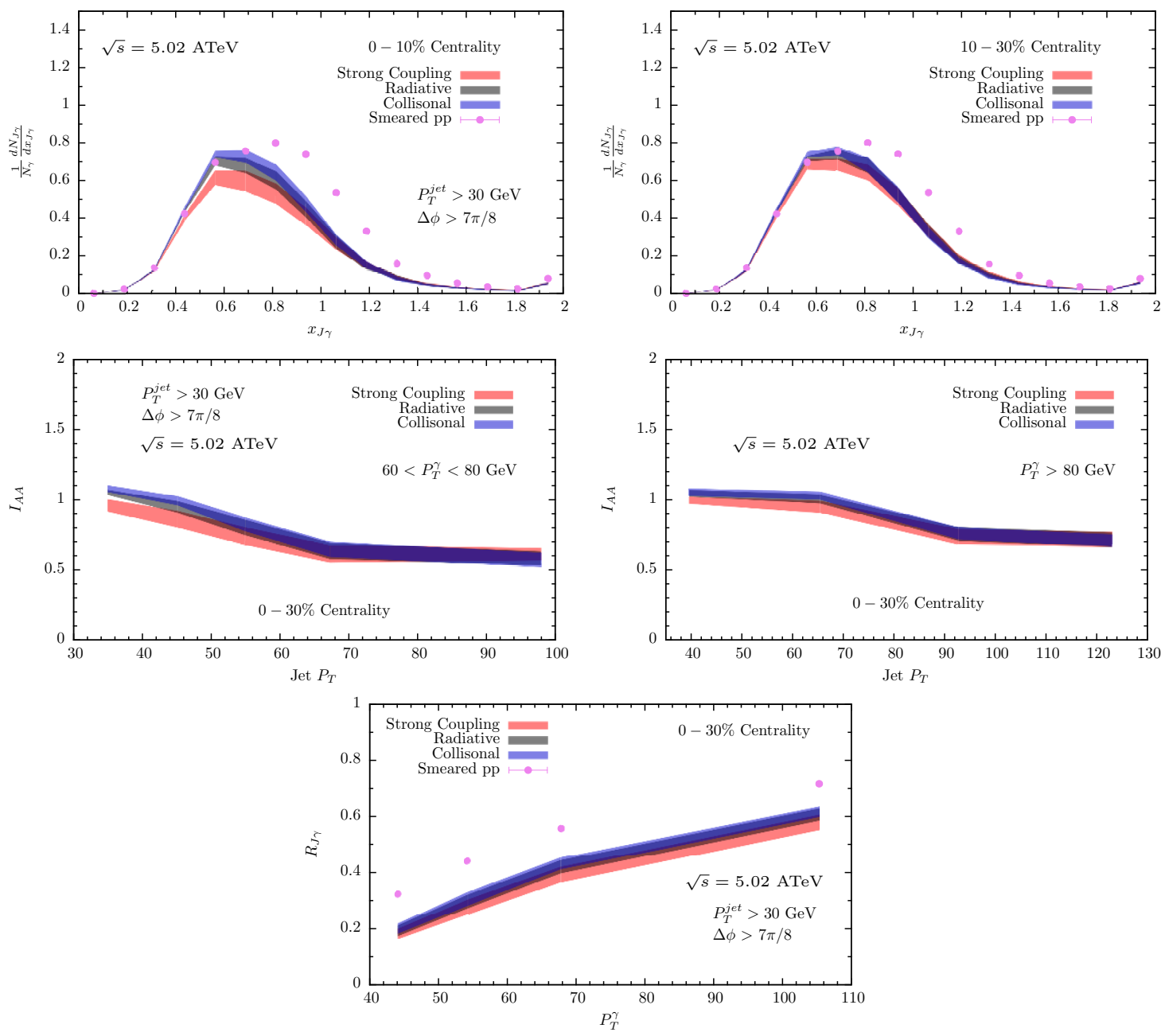

Figure 23. Predictions for several photon-jet observables computed with three different models of the energy loss mechanism in heavy ion collisions with $\sqrt{s}=5.02 \mathrm{ATeV}$. The distributions of the transverse momentum imbalance of photon-jet pairs for two different centralities are displayed in the upper panels. The middle panel shows the ratio of the transverse momentum spectra of jets produced in association with an isolated photon in $\mathrm{Pb}-\mathrm{Pb}$ collisions to that in $\mathrm{p}-\mathrm{p}$ collisions for two different centralities. The lower panel shows the fraction of isolated photons produced in association with a hard jet with $p_{\mathrm{T}}{ }^{\text {jet }}>30 \mathrm{GeV}$ at an azimuthal angle more than $7 \pi / 8$ away from that of the isolated photon. 

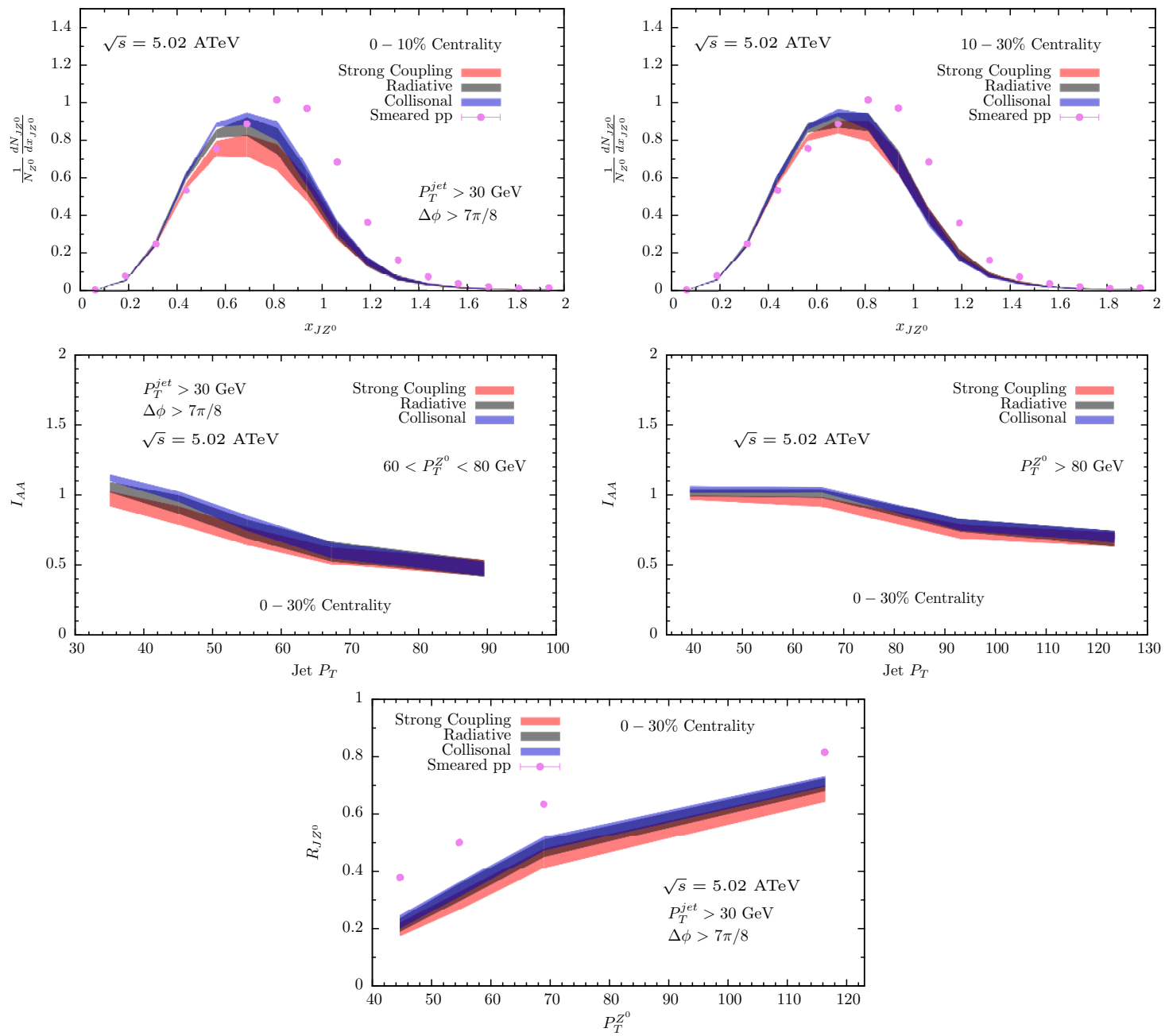

Figure 24. Predictions for several Z-jet observables computed with three different models of the energy loss mechanism in heavy ion collisions with $\sqrt{s}=5.02 \mathrm{ATeV}$. The distributions of the transverse momentum imbalance of Z-jet pairs for two different centralities are displayed in the upper panels. The middle panel shows the ratio of the transverse momentum spectra of jets produced in association with a Z-boson in $\mathrm{Pb}-\mathrm{Pb}$ collisions to that in p-p collisions for two different centralities. The lower panel shows the fraction of Z-bosons produced in association with a hard jet with $p_{\mathrm{T}}{ }^{\text {jet }}>30 \mathrm{GeV}$ at an azimuthal angle more than $7 \pi / 8$ away from that of the isolated photon. 
Open Access. This article is distributed under the terms of the Creative Commons Attribution License (CC-BY 4.0), which permits any use, distribution and reproduction in any medium, provided the original author(s) and source are credited.

\section{References}

[1] PHENIX collaboration, K. Adcox et al., Formation of dense partonic matter in relativistic nucleus-nucleus collisions at RHIC: Experimental evaluation by the PHENIX collaboration, Nucl. Phys. A 757 (2005) 184 [nucl-ex/0410003] [INSPIRE].

[2] BRAHMS collaboration, I. Arsene et al., Quark gluon plasma and color glass condensate at RHIC? The Perspective from the BRAHMS experiment, Nucl. Phys. A 757 (2005) 1 [nucl-ex/0410020] [INSPIRE].

[3] B.B. Back et al., The PHOBOS perspective on discoveries at RHIC, Nucl. Phys. A 757 (2005) 28 [nucl-ex/0410022] [INSPIRE].

[4] STAR collaboration, J. Adams et al., Experimental and theoretical challenges in the search for the quark gluon plasma: The STAR Collaboration's critical assessment of the evidence from RHIC collisions, Nucl. Phys. A 757 (2005) 102 [nucl-ex/0501009] [INSPIRE].

[5] ATLAS collaboration, Observation of a Centrality-Dependent Dijet Asymmetry in Lead-Lead Collisions at $\sqrt{s_{N N}}=2.77 \mathrm{TeV}$ with the ATLAS Detector at the LHC, Phys. Rev. Lett. 105 (2010) 252303 [arXiv:1011.6182] [INSPIRE].

[6] CMS collaboration, Observation and studies of jet quenching in $\mathrm{PbPb}$ collisions at nucleon-nucleon center-of-mass energy $=2.76$ TeV, Phys. Rev. C 84 (2011) 024906 [arXiv:1102.1957] [INSPIRE].

[7] CMS collaboration, Jet momentum dependence of jet quenching in $\mathrm{PbPb}$ collisions at $\sqrt{s_{N N}}=2.76 \mathrm{TeV}$, Phys. Lett. B 712 (2012) 176 [arXiv:1202.5022] [INSPIRE].

[8] CMS collaboration, Studies of jet quenching using isolated-photon+jet correlations in $\mathrm{PbPb}$ and pp collisions at $\sqrt{s_{N N}}=2.76$ TeV, Phys. Lett. B 718 (2013) 773 [arXiv:1205.0206] [INSPIRE].

[9] CMS collaboration, Measurement of jet fragmentation into charged particles in pp and $\mathrm{PbPb}$ collisions at $\sqrt{s_{N N}}=2.76 \mathrm{TeV}$, JHEP 10 (2012) 087 [arXiv:1205.5872] [INSPIRE].

[10] ATLAS collaboration, Measurement of the jet radius and transverse momentum dependence of inclusive jet suppression in lead-lead collisions at $\sqrt{s_{N N}}=2.76 \mathrm{TeV}$ with the ATLAS detector, Phys. Lett. B 719 (2013) 220 [arXiv:1208.1967] [inSPIRE].

[11] ATLAS collaboration, Measurement of the Azimuthal Angle Dependence of Inclusive Jet Yields in $\mathrm{Pb}+\mathrm{Pb}$ Collisions at $\sqrt{s_{N N}}=2.76 \mathrm{TeV}$ with the ATLAS detector, Phys. Rev. Lett. 111 (2013) 152301 [arXiv:1306.6469] [INSPIRE].

[12] CMS collaboration, Modification of jet shapes in PbPb collisions at $\sqrt{s_{N N}}=2.76 \mathrm{TeV}$, Phys. Lett. B 730 (2014) 243 [arXiv: 1310.0878] [INSPIRE].

[13] ALICE collaboration, Measurement of charged jet suppression in $\mathrm{Pb}$-Pb collisions at $\sqrt{s_{N N}}$ = 2.76 TeV, JHEP 03 (2014) 013 [arXiv: 1311.0633] [INSPIRE].

[14] CMS collaboration, Evidence of b-Jet Quenching in PbPb Collisions at $\sqrt{s_{N N}}=2.76 \mathrm{TeV}$, Phys. Rev. Lett. 113 (2014) 132301 [Erratum ibid. 115 (2015) 029903] [arXiv:1312.4198] [INSPIRE]. 
[15] CMS collaboration, Measurement of jet fragmentation in $\mathrm{PbPb}$ and $p p$ collisions at $\sqrt{s_{N N}}=2.76 \mathrm{TeV}$, Phys. Rev. C 90 (2014) 024908 [arXiv: 1406.0932] [INSPIRE].

[16] ATLAS collaboration, Measurement of inclusive jet charged-particle fragmentation functions in $\mathrm{Pb}+\mathrm{Pb}$ collisions at $\sqrt{s_{N N}}=2.76 \mathrm{TeV}$ with the ATLAS detector, Phys. Lett. B 739 (2014) 320 [arXiv:1406. 2979] [INSPIRE].

[17] ATLAS collaboration, Measurements of the Nuclear Modification Factor for Jets in $\mathrm{Pb}+\mathrm{Pb}$ Collisions at $\sqrt{s_{\mathrm{NN}}}=2.76$ TeV with the ATLAS Detector, Phys. Rev. Lett. 114 (2015) 072302 [arXiv: 1411.2357] [INSPIRE].

[18] ALICE collaboration, Measurement of jet suppression in central Pb-Pb collisions at $\sqrt{s_{\mathrm{NN}}}$ = 2.76 TeV, Phys. Lett. B 746 (2015) 1 [arXiv:1502.01689] [InSPIRE].

[19] ALICE collaboration, Measurement of jet quenching with semi-inclusive hadron-jet distributions in central Pb-Pb collisions at $\sqrt{s_{\mathrm{NN}}}=2.76 \mathrm{TeV}$, JHEP 09 (2015) 170 [arXiv: 1506.03984] [INSPIRE].

[20] ATLAS collaboration, Measurement of the production of neighbouring jets in lead-lead collisions at $\sqrt{s_{\mathrm{NN}}}=2.76 \mathrm{TeV}$ with the ATLAS detector, Phys. Lett. B 751 (2015) 376 [arXiv: 1506.08656] [INSPIRE].

[21] PHENIX collaboration, K. Adcox et al., Suppression of hadrons with large transverse momentum in central Au+Au collisions at $\sqrt{s_{N N}}=130-G e V$, Phys. Rev. Lett. 88 (2002) 022301 [nucl-ex/0109003] [INSPIRE].

[22] STAR collaboration, C. Adler et al., Centrality dependence of high $p_{T}$ hadron suppression in Au+Au collisions at $\sqrt{s}_{N N}=130-G e V$, Phys. Rev. Lett. 89 (2002) 202301 [nucl-ex/0206011] [INSPIRE].

[23] J. Casalderrey-Solana, D.C. Gulhan, J.G. Milhano, D. Pablos and K. Rajagopal, A Hybrid Strong/Weak Coupling Approach to Jet Quenching, JHEP 10 (2014) 19 [Erratum ibid. 09 (2015) 175] [arXiv: 1405.3864] [INSPIRE].

[24] J. Casalderrey-Solana, H. Liu, D. Mateos, K. Rajagopal and U.A. Wiedemann, Gauge/String Duality, Hot QCD and Heavy Ion Collisions, arXiv:1101.0618 [INSPIRE].

[25] R. Baier, Y.L. Dokshitzer, A.H. Mueller, S. Peigne and D. Schiff, Radiative energy loss of high-energy quarks and gluons in a finite volume quark-gluon plasma, Nucl. Phys. B 483 (1997) 291 [hep-ph/9607355] [INSPIRE].

[26] R. Baier, Y.L. Dokshitzer, A.H. Mueller and D. Schiff, Medium induced radiative energy loss: Equivalence between the BDMPS and Zakharov formalisms, Nucl. Phys. B 531 (1998) 403 [hep-ph/9804212] [INSPIRE].

[27] M. Gyulassy, P. Levai and I. Vitev, Reaction operator approach to nonAbelian energy loss, Nucl. Phys. B 594 (2001) 371 [nucl-th/0006010] [INSPIRE].

[28] U.A. Wiedemann, Gluon radiation off hard quarks in a nuclear environment: Opacity expansion, Nucl. Phys. B 588 (2000) 303 [hep-ph/0005129] [INSPIRE].

[29] X.-N. Wang and X.-f. Guo, Multiple parton scattering in nuclei: Parton energy loss, Nucl. Phys. A 696 (2001) 788 [hep-ph/0102230] [INSPIRE].

[30] P.B. Arnold, G.D. Moore and L.G. Yaffe, Photon and gluon emission in relativistic plasmas, JHEP 06 (2002) 030 [hep-ph/0204343] [INSPIRE]. 
[31] S. Jeon and G.D. Moore, Energy loss of leading partons in a thermal QCD medium, Phys. Rev. C 71 (2005) 034901 [hep-ph/0309332] [INSPIRE].

[32] P. Jacobs and X.-N. Wang, Matter in extremis: Ultrarelativistic nuclear collisions at RHIC, Prog. Part. Nucl. Phys. 54 (2005) 443 [hep-ph/0405125] [inSPIRE].

[33] S. Turbide, C. Gale, S. Jeon and G.D. Moore, Energy loss of leading hadrons and direct photon production in evolving quark-gluon plasma, Phys. Rev. C 72 (2005) 014906 [hep-ph/0502248] [inSPIRE].

[34] S. Wicks, W. Horowitz, M. Djordjevic and M. Gyulassy, Elastic, inelastic and path length fluctuations in jet tomography, Nucl. Phys. A 784 (2007) 426 [nucl-th/0512076] [INSPIRE].

[35] G.-Y. Qin, J. Ruppert, C. Gale, S. Jeon, G.D. Moore and M.G. Mustafa, Radiative and collisional jet energy loss in the quark-gluon plasma at RHIC, Phys. Rev. Lett. 100 (2008) 072301 [arXiv: 0710.0605] [INSPIRE].

[36] J. Casalderrey-Solana and C.A. Salgado, Introductory lectures on jet quenching in heavy ion collisions, Acta Phys. Polon. B 38 (2007) 3731 [arXiv: 0712.3443] [InSPIRE].

[37] A. Majumder and M. Van Leeuwen, The Theory and Phenomenology of Perturbative QCD Based Jet Quenching, Prog. Part. Nucl. Phys. A 66 (2011) 41 [arXiv:1002.2206] [INSPIRE].

[38] F. D'Eramo, H. Liu and K. Rajagopal, Transverse Momentum Broadening and the Jet Quenching Parameter, Redux, Phys. Rev. D 84 (2011) 065015 [arXiv:1006.1367] [INSPIRE].

[39] Y. Mehtar-Tani, C.A. Salgado and K. Tywoniuk, Anti-angular ordering of gluon radiation in QCD media, Phys. Rev. Lett. 106 (2011) 122002 [arXiv: 1009.2965] [INSPIRE].

[40] Y. Mehtar-Tani, C.A. Salgado and K. Tywoniuk, Jets in QCD Media: From Color Coherence to Decoherence, Phys. Lett. B 707 (2012) 156 [arXiv:1102.4317] [INSPIRE].

[41] G. Ovanesyan and I. Vitev, An effective theory for jet propagation in dense QCD matter: jet broadening and medium-induced bremsstrahlung, JHEP 06 (2011) 080 [arXiv: 1103.1074] [INSPIRE].

[42] J. Casalderrey-Solana and E. Iancu, Interference effects in medium-induced gluon radiation, JHEP 08 (2011) 015 [arXiv:1105.1760] [INSPIRE].

[43] W. Dai, I. Vitev and B.-W. Zhang, Momentum imbalance of isolated photon-tagged jet production at RHIC and LHC, Phys. Rev. Lett. 110 (2013) 142001 [arXiv:1207.5177] [INSPIRE].

[44] F. D'Eramo, M. Lekaveckas, H. Liu and K. Rajagopal, Momentum Broadening in Weakly Coupled quark-gluon Plasma (with a view to finding the quasiparticles within liquid quark-gluon plasma), JHEP 05 (2013) 031 [arXiv:1211.1922] [INSPIRE].

[45] J.-P. Blaizot, E. Iancu and Y. Mehtar-Tani, Medium-induced QCD cascade: democratic branching and wave turbulence, Phys. Rev. Lett. 111 (2013) 052001 [arXiv:1301.6102] [INSPIRE].

[46] Y. Mehtar-Tani, J.G. Milhano and K. Tywoniuk, Jet physics in heavy-ion collisions, Int. J. Mod. Phys. A 28 (2013) 1340013 [arXiv:1302.2579] [InSPIRE].

[47] X.-N. Wang and Y. Zhu, Medium Modification of $\gamma$-jets in High-energy Heavy-ion Collisions, Phys. Rev. Lett. 111 (2013) 062301 [arXiv:1302.5874] [INSPIRE]. 
[48] N. Armesto, H. Ma, M. Martinez, Y. Mehtar-Tani and C.A. Salgado, Coherence Phenomena between Initial and Final State Radiation in a Dense QCD Medium, JHEP 12 (2013) 052 [arXiv: 1308.2186] [inSPIRE].

[49] J.-P. Blaizot, F. Dominguez, E. Iancu and Y. Mehtar-Tani, Probabilistic picture for medium-induced jet evolution, JHEP 06 (2014) 075 [arXiv: 1311.5823] [INSPIRE].

[50] JET collaboration, K.M. Burke et al., Extracting the jet transport coefficient from jet quenching in high-energy heavy-ion collisions, Phys. Rev. C 90 (2014) 014909 [arXiv:1312.5003] [INSPIRE].

[51] Y. Mehtar-Tani and K. Tywoniuk, Jet (de)coherence in Pb-Pb collisions at the LHC, Phys. Lett. B 744 (2015) 284 [arXiv:1401.8293] [INSPIRE].

[52] Z.-B. Kang, R. Lashof-Regas, G. Ovanesyan, P. Saad and I. Vitev, Jet quenching phenomenology from soft-collinear effective theory with Glauber gluons, Phys. Rev. Lett. 114 (2015) 092002 [arXiv: 1405.2612] [INSPIRE].

[53] Y.-T. Chien and I. Vitev, Jet Shape Resummation Using Soft-Collinear Effective Theory, JHEP 12 (2014) 061 [arXiv: 1405.4293] [InSPIRE].

[54] A. Kurkela and U.A. Wiedemann, Picturing perturbative parton cascades in QCD matter, Phys. Lett. B $\mathbf{7 4 0}$ (2015) 172 [arXiv:1407.0293] [InSPIRE].

[55] J.-P. Blaizot, Y. Mehtar-Tani and M.A.C. Torres, Angular structure of the in-medium QCD cascade, Phys. Rev. Lett. 114 (2015) 222002 [arXiv:1407.0326] [INSPIRE].

[56] L. Apolinário, N. Armesto, J.G. Milhano and C.A. Salgado, Medium-induced gluon radiation and colour decoherence beyond the soft approximation, JHEP 02 (2015) 119 [arXiv: 1407.0599] [INSPIRE].

[57] J.-P. Blaizot, L. Fister and Y. Mehtar-Tani, Angular distribution of medium-induced QCD cascades, Nucl. Phys. A 940 (2015) 67 [arXiv:1409.6202] [InSPIRE].

[58] J.-P. Blaizot and Y. Mehtar-Tani, Energy flow along the medium-induced parton cascade, arXiv: 1501.03443 [INSPIRE].

[59] J. Ghiglieri and D. Teaney, Parton energy loss and momentum broadening at NLO in high temperature QCD plasmas, Int. J. Mod. Phys. E 24 (2015) 1530013 [arXiv:1502.03730] [INSPIRE].

[60] C.P. Herzog, A. Karch, P. Kovtun, C. Kozcaz and L.G. Yaffe, Energy loss of a heavy quark moving through $N=4$ supersymmetric Yang-Mills plasma, JHEP 07 (2006) 013 [hep-th/0605158] [INSPIRE].

[61] J. Casalderrey-Solana and D. Teaney, Heavy quark diffusion in strongly coupled $N=4$ Yang-Mills, Phys. Rev. D 74 (2006) 085012 [hep-ph/0605199] [INSPIRE].

[62] S.S. Gubser, Drag force in AdS/CFT, Phys. Rev. D 74 (2006) 126005 [hep-th/0605182] [INSPIRE].

[63] H. Liu, K. Rajagopal and U.A. Wiedemann, An AdS/CFT Calculation of Screening in a Hot Wind, Phys. Rev. Lett. 98 (2007) 182301 [hep-ph/0607062] [INSPIRE].

[64] S.S. Gubser, D.R. Gulotta, S.S. Pufu and F.D. Rocha, Gluon energy loss in the gauge-string duality, JHEP 10 (2008) 052 [arXiv: 0803.1470] [INSPIRE].

[65] Y. Hatta, E. Iancu and A.H. Mueller, Jet evolution in the $N=4$ SYM plasma at strong coupling, JHEP 05 (2008) 037 [arXiv:0803.2481] [INSPIRE]. 
[66] P.M. Chesler, K. Jensen and A. Karch, Jets in strongly-coupled $N=4$ super Yang-Mills theory, Phys. Rev. D 79 (2009) 025021 [arXiv:0804.3110] [InSPIRE].

[67] P.M. Chesler, K. Jensen, A. Karch and L.G. Yaffe, Light quark energy loss in strongly-coupled $N=4$ supersymmetric Yang-Mills plasma, Phys. Rev. D 79 (2009) 125015 [arXiv:0810.1985] [INSPIRE].

[68] P. Arnold and D. Vaman, Jet quenching in hot strongly coupled gauge theories revisited: 3-point correlators with gauge-gravity duality, JHEP 10 (2010) 099 [arXiv:1008.4023] [INSPIRE].

[69] P. Arnold and D. Vaman, Jet quenching in hot strongly coupled gauge theories simplified, JHEP 04 (2011) 027 [arXiv:1101.2689] [INSPIRE].

[70] P. Arnold and D. Vaman, Some new results for 'jet' stopping in AdS/CFT: long version, J. Phys. G 38 (2011) 124175 [arXiv:1106.1680] [InSPIRE].

[71] M. Chernicoff, J.A. Garcia, A. Guijosa and J.F. Pedraza, Holographic Lessons for Quark Dynamics, J. Phys. G 39 (2012) 054002 [arXiv:1111.0872] [InSPIRE].

[72] P.M. Chesler, Y.-Y. Ho and K. Rajagopal, Shining a Gluon Beam Through quark-gluon Plasma, Phys. Rev. D 85 (2012) 126006 [arXiv:1111.1691] [InSPIRE].

[73] P. Arnold, P. Szepietowski and D. Vaman, Coupling dependence of jet quenching in hot strongly-coupled gauge theories, JHEP 07 (2012) 024 [arXiv: 1203.6658] [INSPIRE].

[74] P. Arnold, P. Szepietowski, D. Vaman and G. Wong, Tidal stretching of gravitons into classical strings: application to jet quenching with AdS/CFT, JHEP 02 (2013) 130 [arXiv: 1212.3321] [INSPIRE].

[75] A. Ficnar and S.S. Gubser, Finite momentum at string endpoints, Phys. Rev. D 89 (2014) 026002 [arXiv: 1306.6648] [INSPIRE].

[76] A. Ficnar, S.S. Gubser and M. Gyulassy, Shooting String Holography of Jet Quenching at RHIC and LHC, Phys. Lett. B 738 (2014) 464 [arXiv:1311.6160] [INSPIRE].

[77] P.M. Chesler and K. Rajagopal, Jet quenching in strongly coupled plasma, Phys. Rev. D 90 (2014) 025033 [arXiv: 1402.6756] [InSPIRE].

[78] R. Rougemont, A. Ficnar, S. Finazzo and J. Noronha, Energy loss, equilibration and thermodynamics of a baryon rich strongly coupled quark-gluon plasma, arXiv:1507.06556 [INSPIRE].

[79] W.A. Horowitz and M. Gyulassy, Testing AdS/CFT Drag and pQCD Heavy Quark Energy Loss, J. Phys. G 35 (2008) 104152 [arXiv:0804.4330] [inSPIRE].

[80] C. Marquet and T. Renk, Jet quenching in the strongly-interacting quark-gluon plasma, Phys. Lett. B 685 (2010) 270 [arXiv:0908.0880] [INSPIRE].

[81] W.A. Horowitz and M. Gyulassy, The Surprising Transparency of the sQGP at LHC, Nucl. Phys. A 872 (2011) 265 [arXiv:1104.4958] [INSPIRE].

[82] B. Betz, M. Gyulassy and G. Torrieri, Sensitivity of Azimuthal Jet Tomography to Early Time Energy-Loss at RHIC and LHC, J. Phys. G 38 (2011) 124153 [arXiv:1106.4564] [INSPIRE].

[83] B. Betz and M. Gyulassy, Constraints on the Path-Length Dependence of Jet Quenching in Nuclear Collisions at RHIC and LHC, JHEP 08 (2014) 090 [Erratum ibid. 10 (2014) 043] [arXiv: 1404.6378] [INSPIRE]. 
[84] E. Iancu and A. Mukhopadhyay, A semi-holographic model for heavy-ion collisions, JHEP 06 (2015) 003 [arXiv: 1410.6448] [INSPIRE].

[85] B. Betz and M. Gyulassy, Sensitivity of Pion versus Parton-Jet Nuclear Modification Factors to the Path-Length Dependence of Jet-Energy Loss at RHIC and LHC, Chin. Phys. Lett. 32 (2015) 121204 [arXiv:1503.07671] [INSPIRE].

[86] T. Sjöstrand, S. Mrenna and P.Z. Skands, A Brief Introduction to PYTHIA 8.1, Comput. Phys. Commun. 178 (2008) 852 [arXiv:0710. 3820] [INSPIRE].

[87] C. Shen, Z. Qiu, H. Song, J. Bernhard, S. Bass and U. Heinz, The iEBE-VISHNU code package for relativistic heavy-ion collisions, Comput. Phys. Commun. 199 (2016) 61 [arXiv: 1409.8164] [INSPIRE].

[88] C. Shen, J.-F. Paquet, U. Heinz and C. Gale, Photon Emission from a Momentum Anisotropic quark-gluon Plasma, Phys. Rev. C 91 (2015) 014908 [arXiv:1410.3404] [INSPIRE].

[89] T. Hirano, P. Huovinen and Y. Nara, Elliptic flow in $P b+P b$ collisions at $\sqrt{s_{N N}}=2.76 \mathrm{TeV}$ : hybrid model assessment of the first data, Phys. Rev. C 84 (2011) 011901 [arXiv: 1012.3955] [INSPIRE].

[90] T. Renk, On the sensitivity of the dijet asymmetry to the physics of jet quenching, Phys. Rev. C 85 (2012) 064908 [arXiv: 1202.4579] [INSPIRE].

[91] J. Casalderrey-Solana, J.G. Milhano and P. Quiroga-Arias, Out of Medium Fragmentation from Long-Lived Jet Showers, Phys. Lett. B 710 (2012) 175 [arXiv:1111.0310] [InSPIRE].

[92] D.M. Hofman and J. Maldacena, Conformal collider physics: Energy and charge correlations, JHEP 05 (2008) 012 [arXiv:0803.1467] [INSPIRE].

[93] T. Renk, Parton shower evolution in a 3-D hydrodynamical medium, Phys. Rev. C 78 (2008) 034908 [arXiv:0806.0305] [INSPIRE].

[94] B. Schenke, C. Gale and S. Jeon, MARTINI: an event generator for relativistic heavy-ion collisions, Phys. Rev. C 80 (2009) 054913 [arXiv: 0909.2037] [INSPIRE].

[95] N. Armesto, L. Cunqueiro and C.A. Salgado, Q-PYTHIA: a medium-modified implementation of final state radiation, Eur. Phys. J. C 63 (2009) 679 [arXiv:0907.1014] [INSPIRE].

[96] I.P. Lokhtin, A.V. Belyaev and A.M. Snigirev, Jet quenching pattern at LHC in PYQUEN model, Eur. Phys. J. C 71 (2011) 1650 [arXiv:1103.1853] [INSPIRE].

[97] K.C. Zapp, JEWEL 2.0.0: directions for use, Eur. Phys. J. C 74 (2014) 2762 [arXiv: 1311.0048] [INSPIRE].

[98] K.C. Zapp, Geometrical aspects of jet quenching in JEWEL, Phys. Lett. B 735 (2014) 157 [arXiv: 1312.5536] [inSPIRE].

[99] P.M. Chesler, M. Lekaveckas and K. Rajagopal, Heavy quark energy loss far from equilibrium in a strongly coupled collision, JHEP 10 (2013) 013 [arXiv:1306.0564] [INSPIRE].

[100] M. Lekaveckas and K. Rajagopal, Effects of Fluid Velocity Gradients on Heavy Quark Energy Loss, JHEP 02 (2014) 068 [arXiv:1311.5577] [INSPIRE].

[101] K. Rajagopal and A.V. Sadofyev, Chiral drag force, JHEP 10 (2015) 018 [arXiv: 1505. 07379] [INSPIRE]. 
[102] A. Bazavov et al., Equation of state and QCD transition at finite temperature, Phys. Rev. D 80 (2009) 014504 [arXiv: 0903.4379] [InSPIRE].

[103] CMS collaboration, Nuclear modification factor of high transverse momentum jets in $\mathrm{PbPb}$ collisions at $\sqrt{s_{N N}}=2.76 \mathrm{TeV}$, CMS-PAS-HIN-12-004 (2012).

[104] P. Huovinen and P. Petreczky, QCD Equation of State and Hadron Resonance Gas, Nucl. Phys. A 837 (2010) 26 [arXiv:0912.2541] [INSPIRE].

[105] Y. Aoki, S. Borsányi, S. Dürr, Z. Fodor, S.D. Katz, S. Krieg et al., The QCD transition temperature: results with physical masses in the continuum limit II., JHEP 06 (2009) 088 [arXiv:0903.4155] [INSPIRE].

[106] НотQCD collaboration, A. Bazavov et al., Equation of state in (2+1)-flavor QCD, Phys. Rev. D 90 (2014) 094503 [arXiv: 1407.6387] [InSPIRE].

[107] M.L. Miller, K. Reygers, S.J. Sanders and P. Steinberg, Glauber modeling in high energy nuclear collisions, Ann. Rev. Nucl. Part. Sci. 57 (2007) 205 [nucl-ex/0701025] [INSPIRE].

[108] E. Iancu, The non-linear evolution of jet quenching, JHEP 10 (2014) 95 [arXiv: 1403.1996] [INSPIRE].

[109] J.-P. Blaizot and Y. Mehtar-Tani, Renormalization of the jet-quenching parameter, Nucl. Phys. A 929 (2014) 202 [arXiv:1403.2323] [INSPIRE].

[110] T. Renk, The Rapidity Dependence of Jet Quenching, arXiv:1406.6784 [INSPIRE].

[111] CMS collaboration, Study of isolated photon+jet correlation in $\mathrm{PbPb}$ and pp collisions at $\sqrt{s}_{\mathrm{NN}}=2.76 \mathrm{TeV}$ and $\mathrm{pPb}$ collisions at $\sqrt{s}_{\mathrm{NN}}=5.02 \mathrm{TeV}$, CMS-PAS-HIN-13-006 (2013).

[112] M. Cacciari, G.P. Salam and G. Soyez, FastJet User Manual, Eur. Phys. J. C 72 (2012) 1896 [arXiv: 1111.6097] [INSPIRE].

[113] M. Cacciari, G.P. Salam and G. Soyez, The anti-k $k_{t}$ jet clustering algorithm, JHEP 04 (2008) 063 [arXiv: 0802.1189] [INSPIRE].

[114] Y. Yilmaz, Jet quenching in heavy-ion collisions at LHC with CMS detector, Ph.D. Thesis, Massachusetts Institute of Technology (2013)

[http://web.mit.edu/mithig/theses/Yetkin-Yilmaz-thesis.pdf].

[115] ATLAS collaboration, Measurement of high-pT isolated prompt photons in lead-lead collisions at $\sqrt{s_{N N}}=2.76 \mathrm{TeV}$ with the ATLAS detector at the LHC, ATLAS-CONF-2012-051 (2012).

[116] ATLAS collaboration, Measurement of momentum imbalance in $Z \rightarrow \ell \ell+$ Jet events in Lead-Lead collisions at $\sqrt{s_{N N}}=2.76 \mathrm{TeV}$ with the ATLAS detector, ATLAS-CONF-2012-119 (2012).

[117] ATLAS collaboration, Jet Fragmentation in p+Pb Collisions, ATLAS-CONF-2015-022 (2015).

[118] D. d'Enterria, K.J. Eskola, I. Helenius and H. Paukkunen, Confronting current NLO parton fragmentation functions with inclusive charged-particle spectra at hadron colliders, Nucl. Phys. B 883 (2014) 615 [arXiv:1311.1415] [INSPIRE].

[119] ATLAS collaboration, Measurement of inclusive jet charged particle fragmentation functions in $\mathrm{Pb}+\mathrm{Pb}$ collisions at sqrt $(\mathrm{s}(\mathrm{nn}))=2.76 \mathrm{TeV}$ with the ATLAS detector,

ATLAS-CONF-2012-115 (2012). 
[120] A. Beraudo, J.G. Milhano and U.A. Wiedemann, Medium-induced color flow softens hadronization, Phys. Rev. C 85 (2012) 031901 [arXiv:1109.5025] [INSPIRE].

[121] A. Beraudo, J.G. Milhano and U.A. Wiedemann, The Contribution of Medium-Modified Color Flow to Jet Quenching, JHEP 07 (2012) 144 [arXiv: 1204.4342] [INSPIRE].

[122] P. Aurenche and B.G. Zakharov, Jet color chemistry and anomalous baryon production in AA-collisions, Eur. Phys. J. C 71 (2011) 1829 [arXiv:1109.6819] [InSPIRE].

[123] J. Casalderrey-Solana, E.V. Shuryak and D. Teaney, Conical flow induced by quenched QCD jets, J. Phys. Conf. Ser. 27 (2005) 22 [Nucl. Phys. A 774 (2006) 577] [hep-ph/0411315] [INSPIRE].

[124] P.M. Chesler and L.G. Yaffe, The Wake of a quark moving through a strongly-coupled plasma, Phys. Rev. Lett. 99 (2007) 152001 [arXiv:0706.0368] [INSPIRE].

[125] Y. Tachibana and T. Hirano, Momentum transport away from a jet in an expanding nuclear medium, Phys. Rev. C 90 (2014) 021902 [arXiv: 1402.6469] [InSPIRE].

[126] Y. He, T. Luo, X.-N. Wang and Y. Zhu, Linear Boltzmann Transport for Jet Propagation in the quark-gluon Plasma: Elastic Processes and Medium Recoil, Phys. Rev. C 91 (2015) 054908 [arXiv: 1503.03313] [INSPIRE].

[127] E. Iancu and B. Wu, Thermalization of mini-jets in a quark-gluon plasma, JHEP 10 (2015) 155 [arXiv: 1506.07871] [INSPIRE].

[128] CMS collaboration, Measurement of transverse momentum flow relative to the dijet system in $\mathrm{PbPb}$ and $\mathrm{pp}$ collisions at $\sqrt{s_{N N}}=2.76 \mathrm{TeV}$, CMS-PAS-HIN-14-010 (2014).

[129] J. Casalderrey-Solana, J.G. Milhano and U.A. Wiedemann, Jet Quenching via Jet Collimation, J. Phys. G 38 (2011) 035006 [arXiv:1012.0745] [INSPIRE].

[130] J. Casalderrey-Solana, Y. Mehtar-Tani, C.A. Salgado and K. Tywoniuk, New picture of jet quenching dictated by color coherence, Phys. Lett. B 725 (2013) 357 [arXiv:1210.7765] [INSPIRE]. 